\author{
University of São Paulo \\ "Luiz de Queiroz" College of Agriculture
}

Rotational stocking management on elephant grass for dairy cows: grazing strategies, animal productivity, enteric methane and nitrous oxide emissions

Guilhermo Francklin de Souza Congio

Thesis presented to obtain the degree of Doctor in

Science. Area: Animal Science and Pastures

Piracicaba

2018 


\section{Guilhermo Francklin de Souza Congio \\ Agricultural Engineer}

\section{Rotational stocking management on elephant grass for dairy cows: grazing strategies,}

animal productivity, enteric methane and nitrous oxide emissions

Advisor:

Prof. Dr. SILA CARNEIRO DA SILVA

Thesis presented to obtain the degree of Doctor in Science. Area: Animal Science and Pastures 
Dados Internacionais de Catalogação na Publicação
DIVISÃO DE BIBLIOTECA - DIBD/ESALQ/USP

Congio, Guilhermo Francklin de Souza

Rotational stocking management on elephant grass for dairy cows: grazing strategies, animal productivity, enteric methane and nitrous oxide emissions / Guilhermo Francklin de Souza Congio. - - Piracicaba, 2018.

$107 \mathrm{p}$.

Tese (Doutorado) - - USP / Escola Superior de Agricultura "Luiz de Queiroz".

1. Gases de efeito estufa 2. Gramínea tropical 3. Interceptação luminosa do dossel 4. Manejo do pastejo 5. Qualidade da forragem I. Título 
To my parents Francklin Roberto Leite Congio (in memorian) and Neuza Aparecida de Souza Congio, for their love, support and education

To my sister Ana Carolina de Souza Congio and my nephew 'Greg', for their love and support 


\section{ACKNOWLEDGMENTS}

First I would like to express my deepest gratitude to my family; mom, sister and Greg, for their constant encouragement and understanding. Without your love and support I would have never made it. This thesis is dedicated to you.

To my advisor, Dr. Sila Carneiro da Silva, for his guidance and support during my doctorate program. Above the scientific insights that you provided me, thank you for example of professor and researcher, conducting all activities with professionalism, dedication, and commitment.

Thanks are also due to "Luiz de Queiroz" College of Agriculture - University of São Paulo and the Department of Animal Science that since my undergraduation provided me an unique opportunity that changed the course of my life. To faculty staff of the Department of Animal Science for all training along my journey, specially to Dr. Moacyr Corsi and "Projeto CAPIM" for his tremendous contribution in my formation.

I would also like to thank my guidance committee, in special to Dr. Marília Barbosa Chiavegato, for her constant support all the time and for valuable contributions in reviewing and suggestions for improving the thesis, and Dr. Lilian Elgalise Techio Pereira, for her valuable suggestions.

To researchers Dr. Alexandre Berndt, Dr. Patrícia Perondi Anchão Oliveira, Dr. Rosa Toyoko Shiraishi Frighetto, and to Carlos Eduardo Jordão, Dagmar Oliveira, and Melissa Baccan from EMBRAPA, for their contribution in greenhouse gases sampling and analisys.

To Dr. Pablo Gregorini and Dr. Thomas Maxwell, Faculty of Agricultural and Life Sciences at Lincoln University. Thank you for receiving me as visiting student, for sharing your research, and offering valuable comments towards improving the first published manuscript from my thesis.

My appreciation to the undergraduate students who dedicated few or several weeks helping me in the field and laboratory: Marcel Junqueira Tarraf, Wilton Mourão Filho, João Leonardo Corte Baptistella, Ana Caroline Amorim Krol, Taís Fernandes Landim, Rafaela Aparecida Moraes, Erik Yuri Camargo Barros, João Gabriel Costa Dearo, Rosalie Cuillé, and Felipe Leiber Coelho Pimentel.

My special thanks to friends in my second journey at Piracicaba: Anna Fett, Carolina Aroeira, Eliana Geremia, Fagner Júnior, Guilherme Natsumeda, Guilherme Portes, Larissa Garcia, Max Pasetti, Otávio Almeida, Patrícia Barbosa, and Pedro Guerreiro. I would also like to thank all colleagues from "GEPF" and "LAPF" for scientific discussions and fun moments, and to graduate coleagues for fun moments.

Finally, to the São Paulo State Research Foundation (FAPESP) for funding the project (Process $\mathrm{n}^{\circ}$ 2016/22040-2), and to CNPq and CAPES for providing scholarship during my doctorate program. 


\section{CONTENTS}

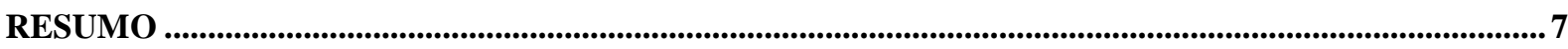

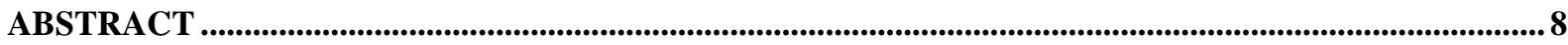

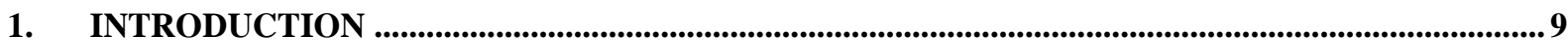

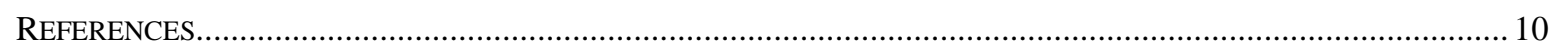

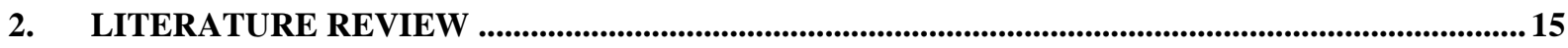

2.1. GRAZING MANAGEMENT AND HERBAGE CHARACTERISTICS ............................................................. 15

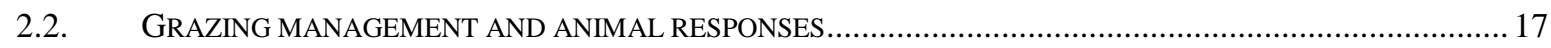

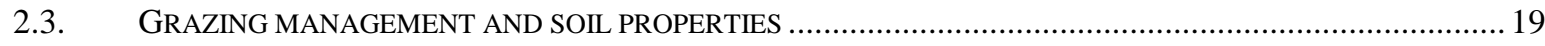

2.4. DIURNAL VARIATION IN HERBAGE CHEMICAL COMPOSITION AND ITS IMPLICATIONS TO PASTURE-

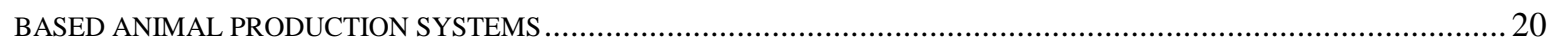

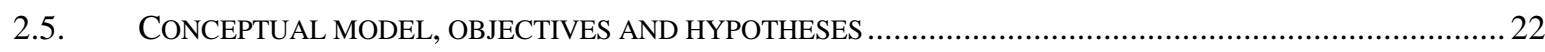

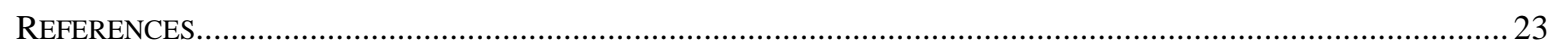

3. STRATEGIC GRAZING MANAGEMENT TOWARDS SUSTAINABLE INTENSIFICATION AT

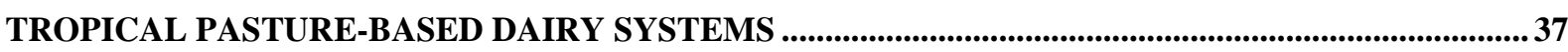

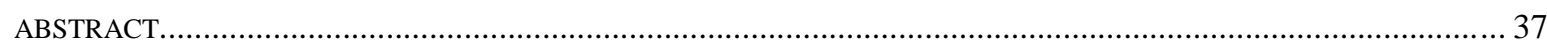

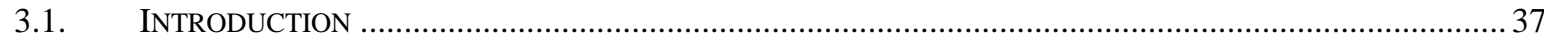

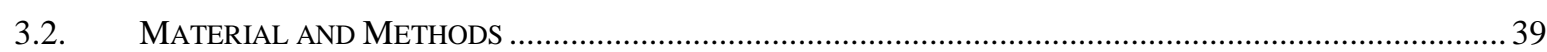

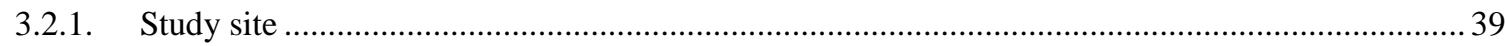

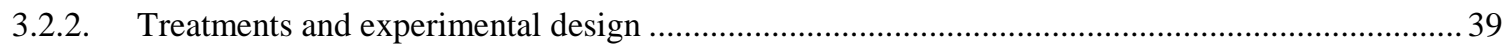

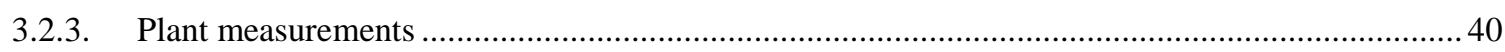

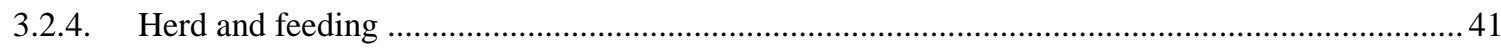

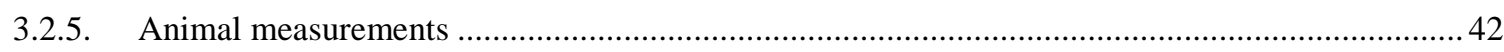

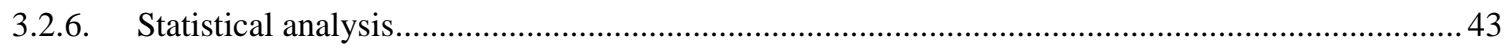

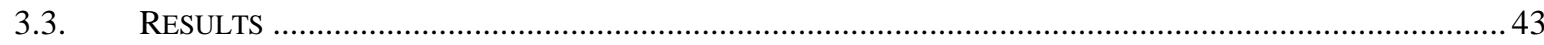

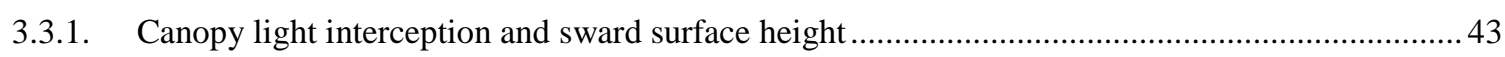

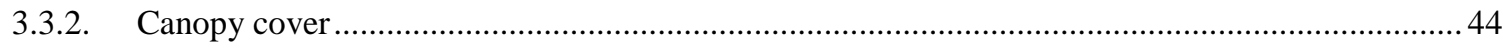

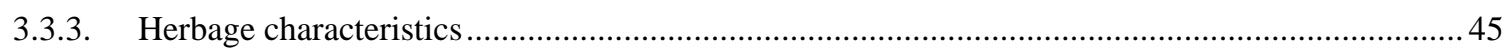

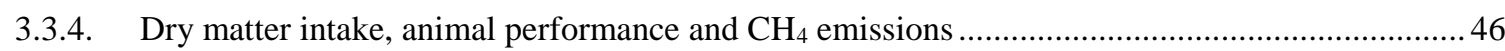

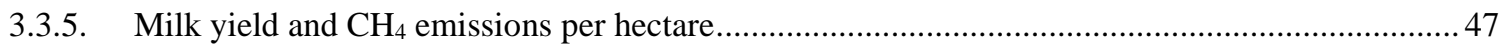

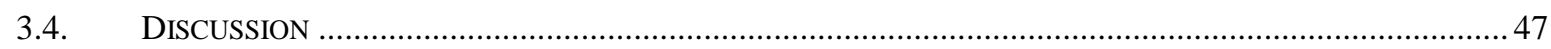

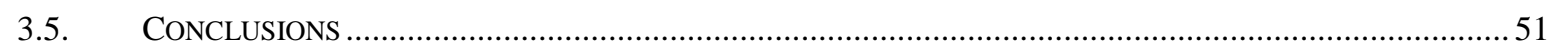

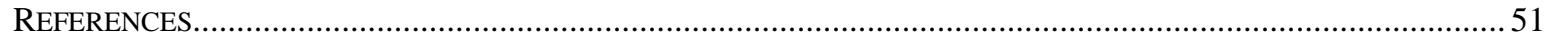

4. STRATEGIC GRAZING MANAGEMENT AND NITROUS OXIDE FLUXES FROM PASTURE SOILS IN TROPICAL DAIRY SYSTEMS …......................................................................................59

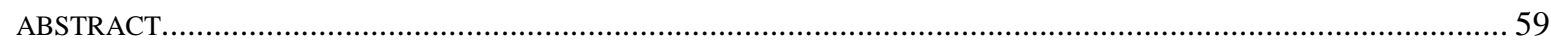

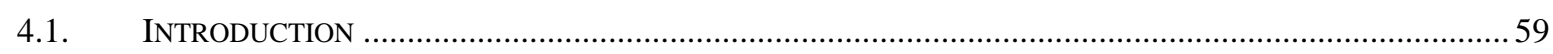

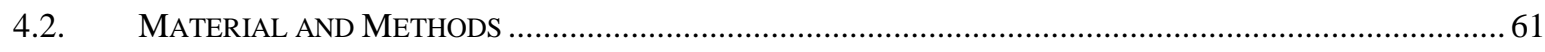

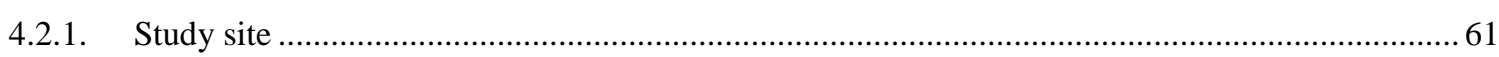




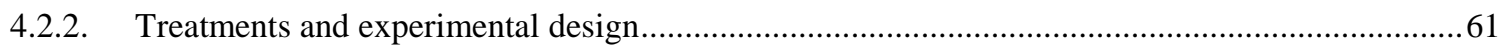

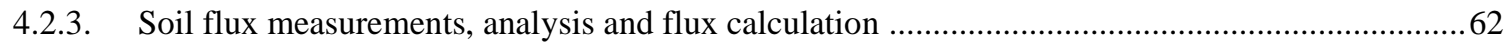

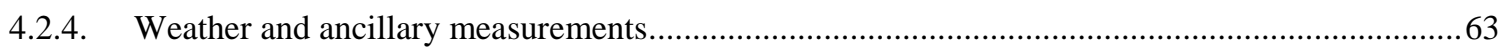

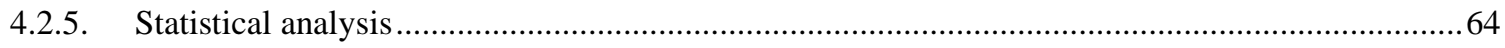

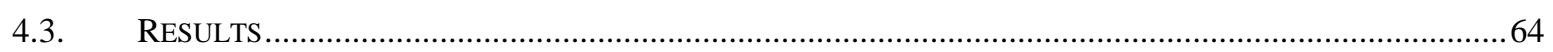

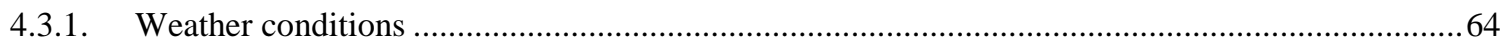

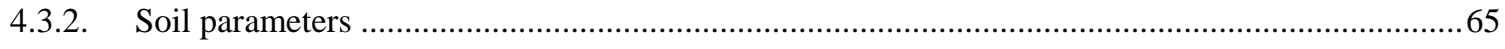

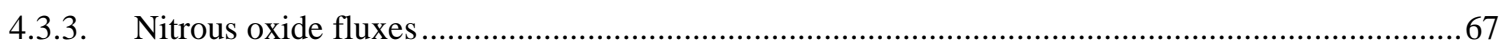

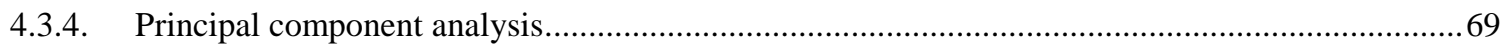

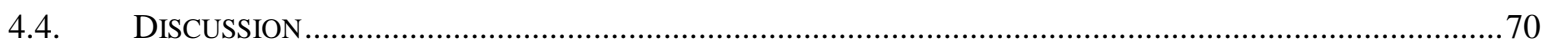

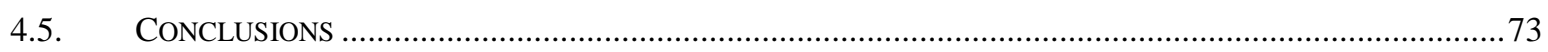

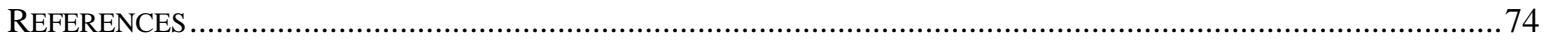

5. EFFECTS OF TIMING OF PADDOCK ALLOCATION ON MILK YIELD AND ENTERIC

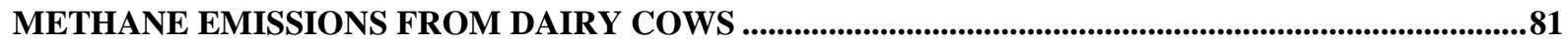

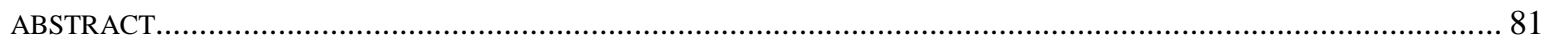

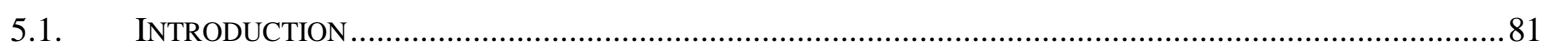

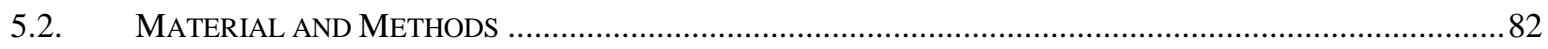

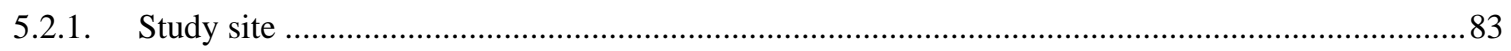

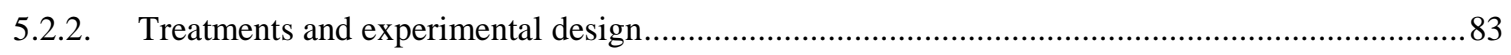

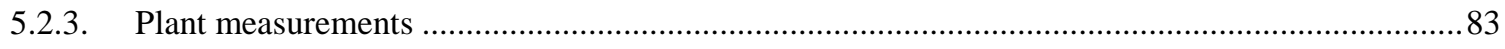

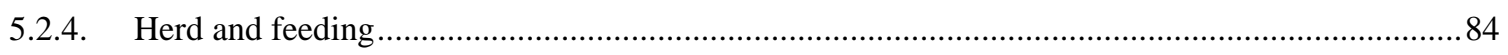

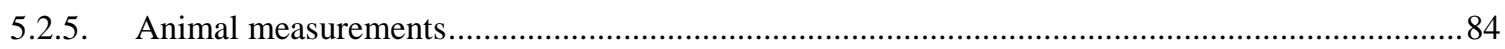

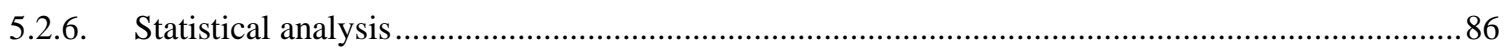

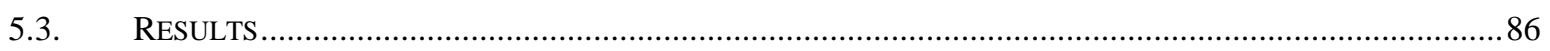

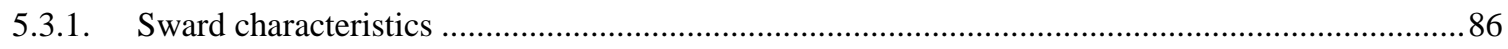

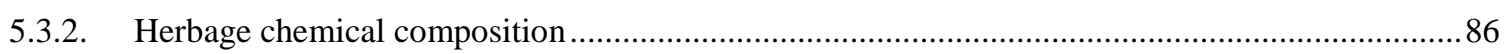

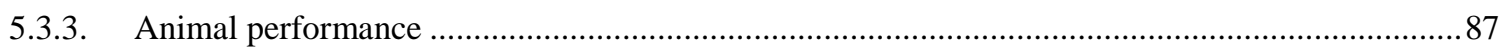

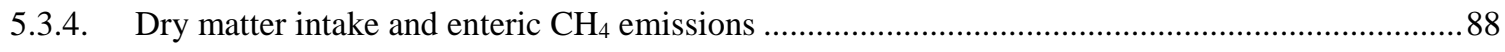

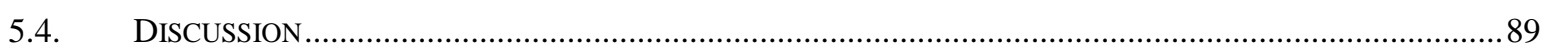

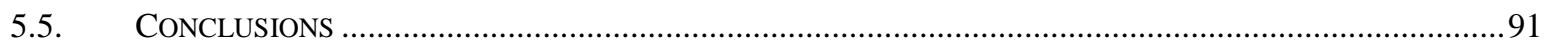

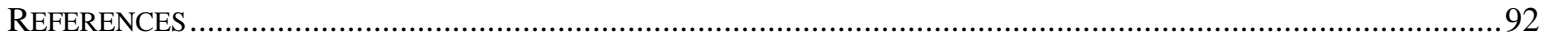

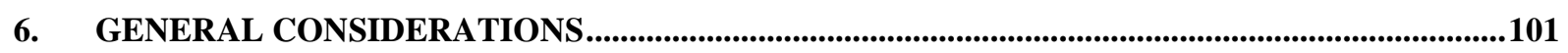

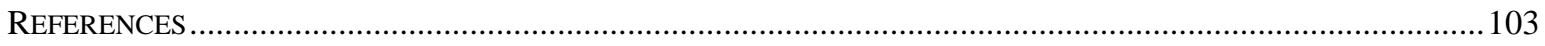

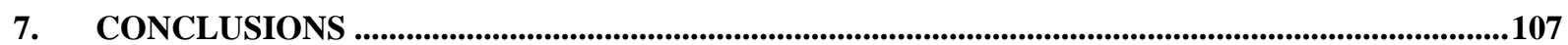




\section{RESUMO}

\section{Pastejo rotativo em capim-elefante para vacas leiteiras: estratégias de pastejo, produtividade animal, emissões de metano entérico e de óxido nitroso}

Sistemas baseados no uso de pastagens são importantes fornecedores de leite para a indústria de latícinios e, dessa forma, terão papel relevante para suportar a crescente demanda por alimentos. No entanto, essa oferta adicional de leite deve ser obtida através de maiores produtividades resultantes da intensificação de sistemas de produção já existentes por meio de estratégias ambientalmente seguras e economicamente rentáveis em direção à intensificação sustentável. A hipótese central deste estudo foi que estratégias simples de manejo do pastejo podem melhorar a eficiência e, ao mesmo tempo, reduzir os principais impactos ambientais dos sistemas de produção animal em pastagens tropicais. Foram realizados dois experimentos em pastagem de capim-elefante (Pennisetum purpureum Schum. Cv. Cameroon) não-irrigada em Piracicaba, SP, Brasil. O objetivo do primeiro experimento foi avaliar a influência de duas metas

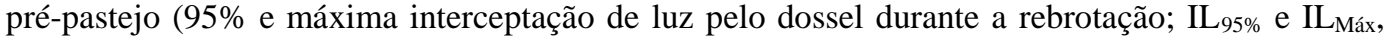
respectivamente) sobre a estrutura do pasto e valor nutritivo da forragem, consumo de matéria seca (CMS), produção de leite, taxa de lotação, emissões de metano entérico $\left(\mathrm{CH}_{4}\right)$ de vacas $\mathrm{HPB} \times$ Jersey, e o fluxo de óxido nitroso dos solos. Os resultados indicaram que a altura pré-pastejo foi maior para $\operatorname{IL}_{\text {Máx }}(\approx 135 \mathrm{~cm})$ do que $\operatorname{IL}_{95 \%}(\approx 100 \mathrm{~cm})$ e pode ser usada como um guia de campo confiável para monitorar a estrutura do pasto. O manejo do pastejo com base nos critérios de IL $95 \%$ melhorou o valor nutritivo da forragem e a eficiência de pastejo, permitindo maior CMS, produção de leite e taxa de lotação. A emissão diária de $\mathrm{CH}_{4}$ entérico não foi afetada; no entanto, as vacas que pastejaram o capim-elefante manejado por IL $95 \%$ foram mais eficientes e emitiram $21 \%$ menos $\mathrm{CH}_{4} / \mathrm{kg}$ de leite e $18 \%$ menos $\mathrm{CH}_{4} / \mathrm{kg}$ de MS consumida. $\mathrm{O}$ aumento de $51 \%$ na produção de leite por hectare superou o aumento de $29 \%$ nas emissões de $\mathrm{CH}_{4}$ entérico por hectare para a meta IL $95 \%$. Os fluxos de óxido nitroso não foram afetados pelas metas pré-pastejo. De maneira geral, o manejo do pastejo com base na meta $\mathrm{IL}_{95 \%}$ é uma prática ambientalmente segura que melhora a eficiência de uso dos recursos alocados por meio da otimização de processos envolvendo plantas, ruminantes e sua interface, e aumenta a eficiência da produção de leite em sistemas baseados em pastagens tropicais. Uma vez que a meta pré-pastejo ideal foi estabelecida durante o primeiro experimento (IL $95 \%$ ), a segunda etapa consistiu-se em um refinamento da primeira. O segundo objetivo foi descrever e medir a influência de dois horários de alocação de novos piquetes aos animais (AM e PM) sobre a composição química da forragem, CMS, produção e composição do leite, e emissões de $\mathrm{CH}_{4}$ entérico de vacas $\mathrm{HPB} \times$ Jersey. Os resultados confirmaram a compreensão geral da variação diurna na composição química da forragem em direção a maiores concentrações de matéria seca e de carboidratos não-fibrosos, e menor concentração de componentes da fibra na forragem amostrada pela à tarde. No entanto, o maior valor nutritivo da forragem da tarde não aumentou o CMS e a produção de leite, nem diminuiu a intensidade de emissão de $\mathrm{CH}_{4}$ das vacas leiteiras. Os resultados também indicaram que a alocação à tarde pode ser uma estratégia de manejo simples e útil que resulta em maior partição de $\mathrm{N}$ para produção de proteína, e menor excreção de $\mathrm{N}$ ureico no leite. A associação da meta pré-pastejo IL $95 \%$ e a alocação do rebanho para um novo piquete à tarde poderia trazer benefícios econômicos, produtivos e ambientais para a intensificação sustentável de sistemas baseados em pastagens tropicais.

Palavras-chave: Gases de efeito estufa; Gramínea tropical; Interceptação luminosa do dossel; Manejo do pastejo; Qualidade da forragem 


\title{
ABSTRACT \\ Rotational stocking management on elephant grass for dairy cows: grazing strategies, animal productivity, enteric methane and nitrous oxide emissions
}

\begin{abstract}
Pasture-based systems are important milk suppliers to dairy industry and thereby will play relevant role to support the growing demand for food. However, this additional milk supply must be obtained through higher yields resulting from intensification of existing farming systems through strategies environmentally friendly and economically profitable towards sustainable intensification. The central hypothesis of this study was that simple grazing management strategies can improve the efficiency while reduce the key environmental issues of tropical pasture-based dairy systems. Two experiments were carried out on a rainfed and non-irrigated elephant grass (Pennisetum purpureum Schum. cv. Cameroon) pasture in Piracicaba, SP, Brazil. The objective of the first experiment was to investigate the influence of two pre-grazing targets (95\% and maximum canopy light interception during pasture regrowth; $\mathrm{LI}_{95 \%}$ and $\mathrm{LI}_{\mathrm{Max}}$, respectively) on sward structure and herbage nutritive value, dry matter intake (DMI), milk yield, stocking rate, enteric methane $\left(\mathrm{CH}_{4}\right)$ emissions by Holstein $\times$ Jersey dairy cows, and nitrous oxide fluxes from the soil. Results indicated that pre-grazing canopy height was greater for $\operatorname{LI}_{\text {Max }}(\approx 135 \mathrm{~cm})$ than $\mathrm{LI}_{95 \%}(\approx 100 \mathrm{~cm})$ and can be used as a reliable field guide for monitoring sward structure. Grazing management based on the $\mathrm{LI}_{95} \%$ target improved herbage nutritive value and grazing efficiency, allowing greater DMI, milk yield and stocking rate by dairy cows. Daily enteric $\mathrm{CH}_{4}$ emission was not affected; however, cows grazing elephant grass at LI I5 $_{5}$ were more efficient and emitted $21 \%$ less $\mathrm{CH}_{4} / \mathrm{kg}$ of milk yield and $18 \%$ less $\mathrm{CH}_{4} / \mathrm{kg}$ of DMI. The $51 \%$ increase in milk yield per hectare overcame the $29 \%$ increase in enteric $\mathrm{CH}_{4}$ emissions per hectare for the $\mathrm{LI}_{95 \%}$ target. Nitrous oxide fluxes were not affected by pre-grazing targets. Overall, strategic grazing management is an environmentally friendly practice that improves the use efficiency of allocated resources through optimization of processes involving plant, ruminant and their interface, and enhances milk production efficiency of tropical pasture-based systems. Once the ideal pre-grazing target was established during he first experiment ( $\mathrm{LI}_{95} \%$, the second step consisted of a refinement of the first phase. The second objective was to describe and measure the influence of two timings of new paddock allocation to cows (AM and PM) on herbage chemical composition and DMI, milk yield, milk compostion, and enteric $\mathrm{CH}_{4}$ emissions of Holstein $\times$ Jersey dairy cows. Results supported the general understanding of diurnal variation in herbage chemical composition towards greater concentrations of dry matter and non-fibrous carbohydrates, and lower concentration of fiber components in the afternoon herbage. However, the higher nutritive value of the afternoon herbage did not result in increasead DMI and milk yield, or decreased intensity of $\mathrm{CH}_{4}$ emission by dairy cows. Our findings also indicate that new paddock allocation in the afternoon can be a simple and useful grazing strategy that results in greater $\mathrm{N}$ partitioning to protein yield, and lower excretion of urea $\mathrm{N}$ in milk. The association of $\mathrm{LI}_{95 \%}$ pre-grazing target and PM allocation could bring economic, productive and environmental benefits towards sustainable intensification of tropical pasture-based systems.
\end{abstract}

Keywords: Greenhouse gases; Tropical grass; Canopy light interception; Grazing management; Herbage quality 


\section{INTRODUCTION}

To meet the world's future food demand, agricultural outputs must grow from 60 to $120 \%$ by 2050 (Godfray et al., 2010; Conforti, 2011; Alexandratos and Bruinsma, 2012) while agriculture environmental footprint must decrease dramatically (Foley et al., 2011). Therefore, food producers are faced with the challenge of supplying food demand through environmentally friendly (Tilman et al., 2002) and economic favorable practices (Foote et al., 2015; Gregorini et al., 2017). In developing countries, agriculture production must increase $80 \%$ through higher yields resulting from intensification of existing agricultural systems (Conforti, 2011). Sustainable intensification was defined as a form of production wherein yields are increased without adverse environmental impact and without the cultivation of more land (Royal Society, 2009). Despite contested (Struik and Kuyper, 2017), this term was deeply discussed (Pretty and Bharucha, 2014) and highlights the needs to increase the productivity (i.e. agricultural product outputs per hectare) of current agricultural systems through practices that minimize key environmental issues (Garnett and Godfray, 2012).

Global warming observed since the mid- $20^{\text {th }}$ century is mostly attributed to anthropic activities that emit greenhouse gases (GHG; IPCC, 2014). Agricultural systems contribute with 10$12 \%$ of global estimated GHG emissions, $50 \%$ of methane $\left(\mathrm{CH}_{4}\right)$ and $60 \%$ of nitrous oxide $\left(\mathrm{N}_{2} \mathrm{O}\right)$ from anthropogenic sources (Smith et al., 2007). Dairy farming systems provide essential high-quality protein that is a major component of human diet (O'Brien et al., 2015; Aguirre-Villegas et al., 2017). However, considering livestock production, they are the second largest contributor accounting for $20 \%$ of total GHG emissions (Gerber et al., 2013). Life cycle assessment approaches reported enteric $\mathrm{CH}_{4}$ and $\mathrm{N}_{2} \mathrm{O}$ from soils as predominant sources of $\mathrm{GHG}$ in dairy farming systems, representing approximately $90 \%$ of total GHG emissions (Aguirre-Villegas et al., 2017). In tropical dairy farming systems, Cunha et al. (2016) reported 53\% for enteric $\mathrm{CH}_{4}$ and $18 \%$ for $\mathrm{N}_{2} \mathrm{O}$ of total GHG emissions for typical Brazilian dairy farms.

Pasture-based systems are important milk suppliers to dairy industry in temperate (Chapman, 2016; Macdonald et al., 2017) and tropical climate (Santos et al., 2014) and thereby will play relevant role to support growing demand (Godfray et al., 2010; Conforti, 2011; Alexandratos and Bruinsma, 2012). The intensification of temperate pasture-based dairy systems has been associated with increasing inputs such as nitrogen fertilizer or imported supplements (Beukes et al., 2012; Foote et al., 2015; Macdonald et al., 2017). However, such intensification practices are associated with issues of environmental concern, namely increased GHG emissions and water degradation (Foley et al., 2011; Vogeler et al., 2013; Foote et al., 2015). Alternatively, grazing management strategies that optimize herbage utilization and digestible dry matter intake by grazing cows could improve land-use and decrease GHG emissions of pasture-based dairy systems (Muñoz et al., 2016; Gregorini et al., 2017).

The key to understanding the principles of grazing management strategies is to comprehend that the harvestable components are photosynthetic organs - predominantly leaves (Parsons et al., 
2011). Studies have reported that grazing management strategies that prioritize leaf accumulation rather than other plant-part components may be useful tools towards efficient pasture-based systems in the tropics (Silveira et al., 2013; Pereira et al., 2014; Da Silva et al., 2015; Da Silva et al., 2017; Sbrissia et al., 2018). Leafy swards mean high herbage quality, since it provides high short-term intake rate by grazing animals, as leaves require less strength to be harvested, and also because they have greater nutritive value than stems and dead material (Trindade et al., 2007; Silva, 2017). In this sense, the development of efficient pasture-based systems with perennial tropical grasses usually focuses on the control of stem elongation and excessive senescence and dead material accumulation by grazing management strategies (Da Silva and Carvalho, 2005; Da Silva et al., 2015).

Although the studies aforementioned have demonstrated the benefits of grazing management strategies, most focused solely on plant responses. There is a knowledge gap relating plant and animal responses and environmental benefits in tropical pasture-based dairy systems. Therefore, the central objective of this study was to investigate the influence of simple grazing management strategies and their effects on the relationships among plant, animal and soil components. The central hypothesis was that simple grazing management strategies optimize processes inherent to plant growth, plant-animal interface, and animal, and provide environmental services, improving efficiency of tropical pasturebased system.

\section{References}

Aguirre-Villegas, H.A., Passos-Fonseca, T.H., Reinemann, D.J., Larson, R.A., 2017. Grazing intensity affects the environmental impact of dairy systems. J. Dairy Sci. 100: 6804-6821. https://doi.org/10.3168/jds.201612325.

Alexandratos, N., Bruinsma, J., 2012. World Agriculture Towards 2030/2050. FAO, Rome http://www.fao.org/fileadmin/templates/esa/Global_persepctives/world_ag_2030_50_2012_rev.pd f.

Beukes, P.C., Scarsbrook, M.R., Gregorini, P., Romera, A.J., Clark, D.A., Catto, W., 2012. The relationship between milk production and farm-gate nitrogen surplus for the Waikato region, New Zealand. J. Environ. Manag. 93:44-51. https://doi.org/10.1016/j.jenvman.2011.08.013.

Chapman, D., 2016. Using ecophysiology to improve farm efficiency: application in temperate dairy grazing systems. Agriculture 6:1-19. https://doi.org/10.3390/agriculture6020017.

Conforti, P., 2011. Looking Ahead in World Food and Agriculture: Perspectives to 2050. Food and Agriculture Organization, Rome. http://www.fao.org/docrep/014/i2280e/i2280e.pdf. 
Cunha, C.S.; Lopes, N.L.; Veloso, C.M.; Jacovine, L.A.G.; Tomich, T.R.; Pereira, L.G.R.; Marcondes, M.I., 2016. Greenhouse gases inventory and carbon balance of two dairy systems obtained two $\begin{array}{llll}\text { methane-estimation } & \text { methods. Sci. Total }\end{array}$ http://dx.doi.org/10.1016/j.scitotenv.2016.07.046.

Da Silva, S.C., Carvalho, P.C.F., 2005. Foraging behaviour and herbage intake in the favourable tropics/subtropics. In: McGilloway, D.A. (Ed.), Grassland: A Global Resource. Wageningen Academic Publishers, Wageningen, The Netherlands :pp. 81-96. http://citeseerx.ist.psu.edu/viewdoc/download?doi=10.1.1.511.4117\&rep=rep1\&type=pdf.

Da Silva, S.C., Chiavegato, M.B., Pena, K.S., Silveira, M.C.T., Barbero, L.M., Junior, S.J.S., Rodrigues, C.S., Limão, V.A., Pereira, L.E.T., 2017. Tillering dynamics of Mulato grass subjected to strategies of rotational grazing management. J. Agric. Sci. 155:1082-1092. https://doi.org/10.1017/S0021859617000223.

Da Silva, S.C., Sbrissia, A.F., Pereira, L.E.T., 2015. Ecophysiology of C4 forage grassesunderstanding plant growth for optimising their use and management. Agriculture 5:598-625. https://doi.org/10.3390/agriculture5030598.

Foley, J.J.A., Ramankutty, N., Brauman, K.A., Cassidy, E.S., Gerber, J.S., Johnston, M.,Mueller, N.D., O'Connell, C., Ray, D.K.,West, P.C., Balzer, C., Bennett, E.M., Carpenter, S.R., Hill, J., Monfreda, C., Polasky, S., Rockström, J., Sheehan, J., Siebert, S., Tilman, D., Zaks, D.P.M., 2011. Solutions for a cultivated planet. Nature 478:337-342. https://doi.org/10.1038/nature10452.

Foote, K.J., Joy, M.K., Death, R.G., 2015. New Zealand dairy farming: milking our environment for all its worth. Environ. Manag. 56:709-720. https://doi.org/10.1007/s00267-015-0517-x.

Garnett, T., Godfray, H.C.J., 2012. Sustainable Intensification in Agriculture: Navigating a Course through Competing Food System Priorities. Food Climate Research Network and the Oxford Martin Programme on the Future of Food. University of Oxford, Oxford. https://www.fcrn.org.uk/sites/default/files/SI report final.pdf.

Gerber, P.J., Steinfeld, H., Henderson, B., Mottet, A., Opio, C., Dijkman, J., Falcucci, A., Ternpio, G., 2013. Tackling Climate Change Through Livestock: a Global Assessment of Emissions and Mitigation Opportunities. Food and Agriculture Organization of the United Nations, pp. 280-286. http://www.fao.org/3/a-i3437e.pdf.

Godfray, H., Beddington, J.R., Crute, I.R., Haddad, L., Lawrence, D., Muir, J.F., Pretty, J., Robinson, S., Thomas, S.M., Toulmin, C., 2010. Food security: the challenge of feeding 9 billion people. Science 327:812-818. https://doi.org/10.1126/science.1185383.

Gregorini, P., Villalba, J.J., Chilibroste, P., Provenza, F.D., 2017. Grazing management: setting the table, designing the menu and influencing the diner. Anim. Prod. Sci. 57(7):1248-1268. http://dx.doi.org/10.1071/AN16637. 
IPCC, 2014. Summary for policymakers. Pages 6-10 in Climate Change 2014: Mitigation of Climate Change. Contribution of Working Group III to the Fifth Assessment Report of the Intergovernmental Panel on Climate Change (IPCC). O. Edenhofer, R. Pichs-Madruga, Y. Sokona, E. Farahani, S. Kadner, K. Seyboth, A. Adler, I. Baum, S. Brunner, P. Eickemeier, B. Kriemann, J. Savolainen, S. Schlomer, C. von Stechow, T. Zwickel, and J.C. Minx, ed. Cambridge University Press, Cambridge, UK. $\quad$ https://www.ipcc.ch/pdf/assessmentreport/ar5/wg3/WGIIIAR5 SPM TS Volume.pdf.

Macdonald, K.A., Penno, J.W., Lancaster, J.A.S., Bryant, A.M., Kidd, J.M., Roche, J.R., 2017. Production and economic responses to intensification of pasture-based dairy production systems. J. Dairy Sci. 100:6602-6619. https://doi.org/10.3168/jds.2016-12497.

Muñoz, C., Letelier, P.A., Ungerfeld, E.M., Morales, J.M., Hube, S., Pérez-Prieto, L.A., 2016. Effects of pre grazing herbage mass in late spring on enteric methane emissions, dry matter intake, and milk production of dairy cows. J. Dairy Sci. 99:7945-7955. https://doi.org/10.3168/jds.2016$\underline{10919 .}$.

O’Brien, D., Shalloo, L., Patton, J., Buckley, F., Grainger, C., Wallace, M., 2012. A life cycle assessment of seasonal grass-based and confinement dairy farms. Agr. Syst. 107:33-46. https://doi.org/10.1016/j.agsy.2011.11.004.

Parsons, A., Rowarth, J., Thornley, J., Newton, P., 2011. Primary production of grasslands, herbage accumulation and use, and impacts of climate change. In: Lemaire G, Hodgson J, Chabbi A (eds) Grassland productivity and ecosystem services, 1st edn. Cabi, Wallingford, UK, pp. 436. https://doi.org/10.1079/9781845938093.A.

Pereira, L.E.T., Paiva, A.J., Geremia, E.V., Da Silva, S.C., 2014. Components of herbage accumulation in elephant grass cvar Napier subjected to strategies of intermittent stocking management. J. Agric. Sci. 152:954-966. https://doi.org/10.1017/S0021859613000695.

Pretty, J., Bharucha, Z.P., 2014. Sustainable intensification in agricultural systems. Ann. Bot. 114:1571-1596. https://doi.org/10.1093/aob/mcu205.

Royal Society, 2009. Reaping the Benefits: Science and the Sustainable Intensification of Global Agriculture. The Royal Society, London. https://royalsociety.org/ /media/Royal Society Content/policy/publications/2009/4294967719.pd f.

Santos, F.A.P., Dorea, J.R.R., de Souza, J., Batistel, F., Costa, D.F.A., 2014. Forage management and methods to improve nutrient intake in grazing cattle. In: Proceedings of the 25th Annual Florida Ruminant Nutrition Symposium. University of Florida, Gainesville, United States of America, pp. 144-164. http://dairy.ifas.ufl.edu/rns/2014/santos.pdf. 
Sbrissia, A.F., Duchini, P.G., Zanini, G.D., Santos, G.T., Padilha, D.A., Schimitt, D., 2018. Defoliation strategies in pastures submitted to intermittent stocking method: underlying mechanisms buffering forage accumulation over a range of grazing heights. Crop Sci. 58:1-10. https://doi.org./10.2135/cropsci2017.07.0447.

Silva, G.P., 2017. Ontogenetic development of Pennisetum purpureum cv. Napier: consequences for grazing management. Thesis, University of São Paulo - Luiz de Queiroz "College of Agriculture". http://www.teses.usp.br/teses/disponiveis/11/11139/tde-28052018-160137/en.php.

Silveira, M.C.T., Da Silva, S.C., Souza Jr., S.J., Barbero, L.M., Rodrigues, C.S., Limão, V.A., Pena, K.S., Nascimento Jr., D., 2013. Herbage accumulation and grazing losses on Mulato grass subjected to strategies of rotational stocking management. Sci. Agric. 70:242-249. https://doi.org/10.1590/S0103-90162013000400004.

Smith, P., Martino, D., Cai, Z., Gwary, D., Janzen, H., Kumar, P., McCarl, B., Ogle, S., O’Mara, F., Rice, C., Scholes, B., Sirotenko, O., 2007. Agriculture. In: Metz, B., Davidson, O.R., Bosch, P.R., Dave, R., Meyer, L.A. (Eds.), Climate change 2007: Mitigation. Contribution of Working Group III to the Fourth Assessment Report of the Intergovernmental Panel on Climate Change. Cambridge University Press, Cambridge, UK and New York, NY, USA. https://www.ipcc.ch/pdf/assessment-report/ar4/wg3/ar4-wg3-chapter8.pdf.

Struik, P.C., Kuyper, T.W., 2017. Sustainable intensification in agriculture: the richer shade of green: a review. Agron. Sustain. Dev. 37 (39). https://doi.org/10.1007/s13593017-0445-7.

Tilman, D., Cassman, K.G., Matson, P.A., Naylor, R., Polasky, S., 2002. Agricultural sustainability and intensive production practices. Nature 418:671-677. https://doi.org/10.1038/nature01014.

Trindade, J.K., Da Silva, S.C., Souza Jr, S.J., Giacomini, A.A., Zeferino, C.V., Guarda, V.D.A., Carvalho, P.C.F., 2007. Composição morfológica da forragem consumida por bovinos de corte durante o rebaixamento do capim-marandu submetido a estratégias de pastejo rotativo. Pesq. Agropec. Bras. 42:883-890. https://doi.org/10.1590/S0100204X2007000600016.

Vogeler, I., Beukes, P.C., Burgraff, V.T., 2013. Evaluation of mitigation strategies for nitrate leaching on pasture-based dairy systems. Agric. Syst. 115:21-28. https://doi.org/10.1016/j.agsy.2012.09.012. 


\section{LITERATURE REVIEW}

\subsection{Grazing management and herbage characteristics}

The pasture management concept involves a wide range of aspects such as the choice of the ideal forage species or mix, liming, nutrient balance and fertilization rate, weed and pest management, soil conservation practices, paddock subdivision, watering system, type and level of supplementation, among others. On the other hand, grazing management is a specific term that refers to monitoring the sward state and controlling the grazing process by grazers through targets that optimize herbage regrowth and animal responses (Da Silva and Corsi, 2003). In continuous stocking grazing management strategies, the question would be at which sward surface height ( $\mathrm{SSH}$ ) the grazer should keep the herbage in order to balance sub-optimal plant and animal responses? In intermittent grazing management strategies (i.e. rotational grazing) the question would be which are the most adequate preand post-grazing heights to achieve the same goals?

Rotational stocking management is widely used in temperate grazing systems and is also being adopted in tropical conditions mainly in dairy farming systems (Santos et al., 2014; Chapman et al., 2016). A large number of studies have been developed to try and understand the most adequate combination between frequency and severity of defoliation for several tropical forage species, or the ideal pre- and post-grazing heights (i.e. frequency and severity, respectively) (Carnevalli et al., 2006; Barbosa et al., 2007; Trindade et al., 2007; Da Silva et al., 2009; Difante et al., 2009a; Difante et al., 2009b; Giacomoni et al., 2009; Difante et al., 2010; Barbosa et al., 2011; Gimenes et al., 2011; Zanini et al., 2012; Silveira et al., 2013; Geremia et al., 2014; Pereira et al., 2014; Pereira et al., 2015a; Pereira et al., 2015b; Silveira et al., 2016; Da Silva et al., 2017; Pereira et al., 2018). The majority of these studies evaluated frequencies based on canopy light interception (LI) combined with severities based in fixed post-grazing heights, and focused on plant responses such as tillering dynamics, morphogenesis, organic reserves, herbage nutritive value, sward structure, and herbage accumulation. These studies observed that tropical grasses regrowth is a function of canopy LI and leaf area index (LAI) with accumulation of herbage fitted to a sigmoid curve with three distinct phases as proposed for temperate swards by Brougham (1955). During the early stages of regrowth, leaves are the main morphological component accumulated. As LAI increases, canopy light intra-competition increases and plants change their growth pattern as a means of optimizing light capture through stem elongation. The shift in growth pattern occurs when canopy LI reaches and exceeds 95\% (LI ${ }_{95 \%}$; Da Silva et al., 2015). These studies have shown systematic relationship between SSH and LI, establishing SSH as a reliable field index for monitoring and controlling herbage regrowth (Da Silva et al., 2015).

Grazing management affects the distribution and arrangement of above-ground plant-part components (i.e. sward structure, Laca and Lemaire, 2000). The frequency of defoliation based on $\mathrm{LI}_{95 \%}$ often minimizes stem elongation of tropical forage, maximizing leaf blade proportion over 
others sward plant-part components (Carnevalli et al., 2006; Barbosa et al., 2007; Trindade et al., 2007; Da Silva et al., 2009). Studies showed that swards managed with the LI $\mathrm{9}_{5 \%}$ target have greater leaf appearance rate, leaf elongation rate, and leaf accumulation in successive grazing cycles than swards managed with the $\mathrm{LI}_{\mathrm{Max}}$ target, which have greater stem elongation and senescence (Barbosa et al., 2011; Pereira et al., 2014; Pereira et al., 2015b; Silveira et al., 2016; Pereira et al., 2018). As a result between leaf growth and senescence rates, $\mathrm{LI}_{95 \%}$ provides greater average net growth rate and is considered the critical LAI to interrupt regrowth under rotational grazing management (Da Silva et al., 2015). Pereira et al. (2015a) also reported changes in horizontal sward structure as a function of grazing management. The $\mathrm{LI}_{95} \%$ target provided greater soil cover by elephant grass tussocks (Pennisetum purpureum Schum. cv. Napier). Furthermore, the exacerbated competition for light for the $\mathrm{LI}_{\mathrm{Max}}$ target resulted in tiller death, reduced tillering, and less stability of plant population impairing pasture persistence (Pereira et al., 2015a).

Herbage chemical composition is a function of the proportion of plant-part components in the herbage mass and their tissue anatomy (Moore, 1994). Stems contain higher proportion of cell wall tissues and less photosynthetic tissues than leaves (Wilson and Kennedy, 1996). On the other hand, most protein compounds are present in leaves, with the majority associated with photosynthetic enzymes (Gastal and Durand, 2000). As a consequence of changes in plant-part components in the grazing strata, the frequency of defoliation associated with the $\mathrm{LI}_{95 \%}$ target is an efficient tool to improve herbage nutritive value in tropical grasses (Trindade et al., 2007). Studies reported lower acid-detergent fiber and greater crude protein concentrations for elephant grass (Pennisetum purpureum Schum) (Voltolini et al., 2010a; Geremia et al., 2014), and greater in vitro digestible organic matter for signal grass (Brachiaria decumbens cv. Basilisk, syn. Urochloa decumbens Stapf R. D. Webster) (Pedreira et al., 2017) managed with the $\mathrm{LI}_{95 \%}$ rather than the $\mathrm{LI}_{\mathrm{Max}}$ target.

Efficient pasture-based systems should maximize the proportion of consumed relatively to produced herbage (Chapman et al., 2016). In order to do that, they have to prioritize leaf accumulation and increase grazing efficiency or herbage utilization through reduced losses by cattle trampling and plant senescence (Da Silva et al., 2015; Chapman et al., 2016). Several studies have shown greater senescence for tropical grasses managed with the $\mathrm{LI}_{\mathrm{Max}}$ compared to the $\mathrm{LI} 95 \%$ target because of the longer regrowth intervals (Barbosa et al., 2011; Pereira et al., 2014; Pereira et al., 2015b; Silveira et al., 2016; Pereira et al., 2018). Longer regrowth intervals usually result in taller swards with high pregrazing herbage mass (Da Silva et al., 2009; Pereira et al., 2015b) which are more susceptible to losses by cattle trampling (Carnevalli et al., 2006; Silveira et al., 2013). Both greater senescence and grazing losses by cattle trampling contribute to dereased grazing efficiency of taller swards managed with the $\mathrm{LI}_{\text {Max }}$ compared to the $\mathrm{LI}_{95 \%}$ target.

Studies showing the numerous benefits on plant growth managed with the $\mathrm{LI}_{95} \%$ target were mostly compared with management using the $\mathrm{LI}_{\mathrm{Max}}$ target (early known as $\mathrm{LI}_{100 \%}$ ). Recently, Sbrissia et al. (2018) assessing a range of LI targets lower than 95\%, highlighted a new opportunity for tropical 
forage grasses under rotational stocking management. They suggested that there is a range of pregrazing heights with no impact on net herbage accumulation rate, as long as the defoliation used is moderate (removal of no more than $50 \%$ of the initial pre-grazing height). The authors explained that the same homeostatic mechanisms that buffer herbage accumulation across a range of targets in continuously stocked swards can be applied to rotationally stocked swards. If more studies corroborate these responses for different tropical grasses, farmers would have a flexible optimal range to manage their pastures where $\mathrm{LI}_{95} \%$ would be the upper threshold to interrupt sward regrowth.

Regarding severity of defoliation, studies that assessed mainly plant responses based on fixed residual post-grazing heights usually observed that greater severities (i.e. lower post-grazing heights) were positively related to herbage accumulation and grazing efficiency, and negatively related to nutritive value of the consumed herbage (Carnevalli et al., 2006; Barbosa et al., 2007; Difante et al., 2009b). However, using the concept of severity of defoliation as a percentage of initial pre-grazing height and having the grazing animal under perspective, studies have shown that levels of defoliation until $40-50 \%$ of the pre-grazing height result in relatively stable and high rate of short-term herbage intake (Fonseca et al., 2012; Fonseca et al., 2013; Carvalho, 2013; Mezzalira et al., 2014). They reported that beyond this herbage depletion level preferred leaves become scarce and stem and dead material become predominant in succeeding lower pasture layers impairing the efficiency of nutrient harvesting per unit of bite (Carvalho, 2013). According to Zanini et al. (2012), regardless of forage species and pre-grazing height, $90 \%$ of stem is present in the lower half of the canopy.

\subsection{Grazing management and animal responses}

Defoliation strategies change tissue turnover, photosynthates allocation pattern, and finally the rate of processes related to morphogenetic characteristics that, in turn, determine sward structural characteristics (Chapman and Lemaire, 1993). As detailed in the previous session, the pre-grazing target of $\mathrm{LI}_{95 \%}$ under rotational grazing management optimizes harvestable plant-part components (i.e. leaves) rather than support morphological components (i.e. stems) and dead material, which are plantpart components avoided by grazers (Trindade et al., 2007). Furthermore, grazing losses by cattle trampling are reduced with grazing at $\mathrm{LI}_{95 \%}$ compared to $\mathrm{LI}_{\text {Max. }}$ As a result of greater leaf accumulation and lower losses by trampling and senescence, the $\mathrm{LI}_{95 \%}$ target provides more feed per hectare supporting higher stocking rates. Voltolini et al. (2010b) and Gimenes et al. (2011) found stocking rate increases ranging from $10 \%$ to $42 \%$ in elephant and palisade grass pastures managed with the $\mathrm{LI}_{95 \%}$ relative to the $\mathrm{LI}_{\mathrm{Max}}$ target.

Daily herbage intake is determined by interactions between sward structure and grazing animals (Wade and Carvalho, 2000). Poppi et al. (1987) suggested that herbage intake follows an asymptotic distribution represented by two distinct phases. In the first ascending phase, herbage intake 
is related to sward structure (i.e. herbage or leaf mass, pre-SSH, leaf-to-stem ratio) and grazing behavior (i.e. grazing time, diet selection, bite mass and bite rate), which are characteristics strongly affected by grazing management strategies (Da Silva and Carvalho, 2005). In the second asymptotic phase, nutritional factors such as herbage chemical composition, digesta retention time in the rumen and concentration of metabolic compounds are more relevant in controlling intake (Poppi et al., 1987). Swards constantly kept at taller heights (such as those managed with the $\mathrm{LI}_{\text {Max }}$ target) result in lower short-term intake rate owing to the excessive length of leaf blade and lower bulk density of herbage in the upper strata (Palhano et al., 2007; Fonseca et al., 2013; Carvalho, 2013). At the rumen level, more fibrous herbage (i.e. higher NDF, ADF and lignin) is associated with greater ruminal retention time, lower fermentation and passage rate, and lower herbage intake (Mertens, 1994; Allen, 1996; Allen, 2000; Forbes, 2007). On the other hand, leafy swards with high herbage nutritive value as those resulting from management with the $\mathrm{L}_{95}$ target would optimize animal grazing behavior and rumen fill in order to achieve high daily herbage intake. It is worth mentioning that frequencies of defoliation based on fixed-length rest periods in an attempt to easy operationalize the herd management into setpaddock area are unable to adequately control sward structure and usually result in decreased animal performance and animal productivity (Pedreira et al., 2009; Voltolini et al., 2010b; Euclides et al., 2014).

The severity of defoliation can also affect grazing behavior and nutritive value of the consumed herbage (Difante et al., 2009b; Fonseca et al., 2012). Fonseca et al. (2012) reported that severities of defoliation greater than $40-50 \%$ removal of pre-grazing height resulted in linear decrease of the short-term rate of herbage intake jeopardizing daily herbage intake and animal performance. At the same time, severities of defoliation greater than the ones proposed by Fonseca et al. (2012) would optimize grazing efficiency and stocking rate (Difante et al., 2009a). Thus, there is a clear trade-off between animal performance and stocking rate as proposed early by Mott (1960), and the most productive grazing strategy should be one able to conciliate significant levels of animal performance with the highest possible stocking rate. Recent approaches have shown that defoliation levels around $45 \%$ of pre-grazing height can increase in $68 \%$ animal performance coupled with stocking rate reductions of around 30\% (Euclides et al., 2015; Euclides et al., 2018). Therefore, grazing management strategies that associate the $\mathrm{LI}_{95} \%$ pre-grazing target with moderate levels of defoliation (not exceeding the removal of 50\% of the pre-grazing height) seem to be more appropriate to achieve higher levels of animal productivity.

However, at the present time, environmental concern is undividable from successful and productive animal production systems (Chiavegato et al., 2018). Greenhouse gases (GHG) emissions are estimated to be the most significant among all categories of environmental impacts in livestock farming systems (O’Brien et al., 2012; Guerci et al., 2013; Gregorini et al, 2016), and enteric methane $\left(\mathrm{CH}_{4}\right)$ represents more than $80 \%$ of total $\mathrm{GHG}$ emissions in pasture-based dairy farming systems (Aguirre-Villegas et al., 2017). Enteric $\mathrm{CH}_{4}$ production from animal digestion is affected by the 
amount and nature of feed, and the extent of its degradation, which in turn determines the amount of hydrogen formed in the rumen (Janssen, 2010). The model proposed by Janssen (2010) suggests that greater digesta passage rates increase hydrogen concentration in the rumen. Consequently, microorganisms would select pathways thermodynamically more favorable to this condition, which produce less hydrogen resulting in less $\mathrm{CH}_{4}$ formed per unit of feed ingested (i.e. $\mathrm{CH}_{4}$ yield). Studies carried out in temperate grazing systems have shown that pre-grazing height of typical ryegrass $x$ white clover mixed pastures can be an important tool to mitigate enteric $\mathrm{CH}_{4}$ emissions from pasturebased farming systems. These studies reported no differences in daily enteric $\mathrm{CH}_{4}$ emissions from beef heifers (Boland et al., 2013) and dairy cows (Wims et al., 2010; Muñoz et al., 2016) grazing low versus high herbage mass swards, even with significant differences reported in daily herbage intake and herbage nutritive value. However, they observed reductions on $\mathrm{CH}_{4}$ yield and $\mathrm{CH}_{4}$ emission intensity (i.e $\mathrm{CH}_{4}$ per unit of final product) from cows grazing low versus high herbage mass swards (Wims et al., 2010; Boland et al., 2013; Muñoz et al., 2016).

\subsection{Grazing management and soil properties}

Grazing management strategies can strongly affect processes related to plant growth (Da Silva et al., 2015), animal ingestive behavior (Da Silva and Carvalho, 2005), and soil characteristics (de Klein et al., 2008; Luo et al., 2017). Frequency of defoliation based on the $\mathrm{LI}_{95 \%}$ target increases leaf accumulation and reduces litter deposition owing to decreased senescence and grazing losses by cattle trampling, compared with the $\mathrm{LI}_{\mathrm{Max}}$ target. As a consequence, studies have shown that the $\mathrm{LI}_{95 \%}$ target provides more feed per hectare supporting up to $42 \%$ increase in stocking rate (Voltolini et al., 2010b). Higher stocking rates modify soil properties (i.e. bulk density, moisture, temperature, $\mathrm{pH}$, aeration) (Warren et al., 1986; Silva et al., 2003; Schmalz et al., 2013) and increase nitrogen (N) discharge to soil through more frequent deposition of urine and feces patches on paddocks (de Klein et al., 2008). These factors, in turn, change microbial community growth and activity (Bardgett et al., 1996; Bardgett et al., 2001; Bardgett and Wardle, 2003) and determine the intensity of processes associated to nitrous oxide $\left(\mathrm{N}_{2} \mathrm{O}\right)$ flux derived from soils (de Klein et al., 2008; Levine et al., 2011; Luo et al., 2017).

Nitrous oxide is the main GHG from soil and the second most representative between all GHG, ranging from $15 \%$ (housed) to $25 \%$ (pasture-based) of total GHG emissions in dairy farming systems (Aguirre-Villegas et al., 2017). Nitrous oxide is formed through microbial transformation of $\mathrm{N}$ compounds in the soil, typically by incomplete denitrification or by nitrification (Wrage et al., 2001; Saggar et al., 2013). Nitrification is an aerobic process where soil microbials oxidise $\mathrm{NH}_{4}{ }^{+}$to $\mathrm{NO}_{3}{ }^{-}$ and $\mathrm{N}_{2} \mathrm{O}$ is formed through chemical decomposition of intermediates, while denitrification is an anaerobic process where $\mathrm{NO}_{3}{ }^{-}$is reduced into $\mathrm{N}_{2}$, with $\mathrm{N}_{2} \mathrm{O}$ an obligatory intermediate (Wrage et al., 
2001; de Klein and Eckard, 2008). Nitrous oxide fluxes are affected by a wide range of proximal and distal regulators, making its regulation a very complex process (de Klein et al., 2008; Luo et al., 2017). Proximal soil factors include mineral nitrogen $\left(\mathrm{NH}_{4}{ }^{+}\right.$and $\left.\mathrm{NO}_{3}{ }^{-}\right)$and organic carbon availabilities, moisture, $\mathrm{pH}$, temperature, and texture that are, in turn, affected by distal regulators such as rainfall or irrigation, soil compaction, organic matter and $\mathrm{N}$ inputs (de Klein et al., 2008; Luo et al., 2017). In grazed pastoral soils, the key drivers related to $\mathrm{N}_{2} \mathrm{O}$ fluxes are $\mathrm{N}$ inputs (i.e. excreta and fertilizer) and soil aeration (i.e. water-filled pore space, WFPS) (de Klein et al., 2008; Luo et al., 2017). Periods when soil characteristics favorable to $\mathrm{N}_{2} \mathrm{O}$ production coincide are called "hot moments" (Luo et al., 2017). In tropical conditions, these "hot moments" usually occur during late spring and summer when pastures are intensively growing owing to the abundance of solar radiation, rainfall, and $\mathrm{N}$ inputs.

The majority of studies involving $\mathrm{N}_{2} \mathrm{O}$ flux from pasture soils have assessed the effects of proximal factors on processes and emission factors in temperate conditions (Saggar et al., 2013; de Klein et al., 2014; Barneze et al., 2015; Venterea et al., 2015; Gardiner et al., 2016; Samad et al., 2016; Clough et al., 2017; Gardiner et al., 2017; van der Weerden et al. 2017; Luo et al., 2018; Rex et al., 2018). The little information available for tropical pastures has also focused on nitrous oxide fluxes related to proximal factors within urine patches (Barneze et al., 2014; Lessa et al., 2014; Mazzetto et al., 2014; Mazzetto et al., 2015). There is no information available regarding $\mathrm{N}_{2} \mathrm{O}$ fluxes from soils of tropical pasture-based dairy farming systems, as influenced by grazing management strategies. In fact, farming scale studies are scarce even in temperate conditions. Previous results have shown that intensively managed grasslands are stronger sources of $\mathrm{N}_{2} \mathrm{O}$ than extensively managed grasslands owing to greater inputs of $\mathrm{N}$ fertilizer and excreta (Smith et al., 2001; Flechard et al., 2007; Rafique et al., 2011). However, they have not accounted for animal outputs that are usually greater in intensively managed systems and could compensate the higher $\mathrm{N}_{2} \mathrm{O}$ fluxes.

\subsection{Diurnal variation in herbage chemical composition and its implications to pasture- based animal production systems}

Several studies have reported diurnal variations in herbage chemical composition (Lechtenberg et al., 1971; Orr et al., 1997; Ciavarella et al., 2000; Griggs et al., 2005; Gregorini et al., 2006; Shewmaker et al., 2006; Gregorini et al., 2008; Morin et al., 2011; De Oliveira et al., 2018). Such variation is mainly related to the balance between the photosynthesis and respiration processes coupled with water loss through plant transpiration (Gregorini, 2012). Photosynthetic activity occurs in chloroplasts mainly in the leaves, and when synthesis of carbohydrates exceeds their use the surplus may be temporarily stored in organs present in leaves and stems (Perry and Moser, 1974; Parsons et al., 1983). Sucrose and fructans are the predominant carbohydrate constituents of temperate grasses (i.e. $\mathrm{C}_{3}$ metabolism), while sucrose and starch are typical in tropical grasses (i.e. $\mathrm{C}_{4}$ metabolism; 
White, 1973; Chatterton et al., 1989; Pollock and Cairns, 1991). The surplus carbohydrate stored inside the chloroplasts during the day is known as transitory and is used as a source of carbon to plant respiration at night (Lu et al., 2005; Zeeman et al., 2007; Weise et al., 2011).

The balance between these processes leads to non-fibrous carbohydrate (NFC) and dry matter (DM) concentration increases from dawn to dusk, reaching greatest concentrations between 12 13h after sunrise (Lechtenberg et al., 1971; Morin et al., 2011; Morin et al., 2012; De Oliveira et al., 2018). The increase of NFC occurs mainly in the upper layers of sward owing to greater proportion of leaves rather than other plant-part components (Delagarde et al., 2000; De Oliveira et al., 2018). For temperate swards, including grass and legumes, Pelletier et al. (2010) reported increases of soluble carbohydrates (SC) from 6 to 105\% for PM herbage compared to AM herbage; however, most results reported mean increases around 50\% (Ciavarella et al., 2000; Mayland et al., 2000; Pelletier et al., 2010; Vasta et al., 2012; Pulido et al., 2015; Vibart et al., 2017). Increases in starch have been reported around 100\% for PM temperate legumes (Orr et al., 1997; Brito et al., 2008; Pelletier et al., 2010; Andueza et al., 2012) and 30\% for PM temperate grass swards (Orr et al., 1997; Bertrand et al., 2008; Pelletier et al., 2010; Brito et al., 2016). Regarding DM concentration, most literature reported increases from 14 up to 27\% (Ciavarella et al., 2000; Delagarde et al., 2000; Trevaskis et al., 2001; Gregorini et al., 2008; Abrahamse et al., 2009; De Oliveira et al., 2014; Pulido et al., 2015; Vibart et al., 2017). Gregorini et al. (2009) explained that the diurnal changes in temperature, solar radiation, and relative humidity, coupled with accumulation of photosynthates may explain the increase in DM concentration from AM to PM.

The increase in NFC and DM concentrations during the day often dilutes other nutritional entities (Gregorini et al., 2009; Gregorini, 2012; Vibart et al., 2017). Studies have reported decreases in fiber concentration (Orr et al., 2001; Burns et al., 2007; Abrahamse et al., 2009) associated to greater digestibility (Burns et al., 2007; Pelletier et al., 2010) for PM temperate swards. Considering protein fractions, studies have reported decrease for PM compared to AM herbage (De Oliveira et al., 2014; Pulido et al., 2015; Vibart et al., 2017) while other showed no effect (Gregorini et al., 2008; Delagarde et al., 2000; Fisher et al., 2002). In fact, greater concentrations of SC and starch in the afternoon improve the NFC/protein ratio which optimize the supply of energy and protein to rumen microorganisms (Bryant et al., 2012; Bryant et al., 2014) reducing urinary-N discharges onto pastures (Gregorini et al., 2010; Gregorini, 2012; Vibart et al., 2017). Moreover, Gregorini et al. (2009) observed that diurnal increases of herbage DM and NFC concentrations, associated with dilution of fiber concentration diminished leaf toughness and reduced particle size from AM to PM. The fluctuations in chemical composition, toughness and particle size mean that herbage feeding value (i.e. herbage quality) is highest during the afternoon and early evening (Gregorini, 2012).

Daily herbage intake of grazing ruminants is described by the cumulative outcome of all meals (i.e. grazing events) during the day (Gibb, 2007). Studies have reported three to five grazing events during cooler parts of the day (Gregorini, 2012). However, regardless of the frequency, the 
major grazing events occur early in the morning and late in the afternoon/early evening for sheep (Orr et al., 1997), beef cattle (Gregorini et al. 2007), and dairy cows (Gibb et al. 1998). The temporal pattern of herbage intake, ingestive and digestive behavior of grazing ruminants can be altered by timing of animal allocation to new strips or paddocks when subjected to intermittent stocking management (Gibb et al., 1998; Orr et al., 2001; Gregorini et al., 2006; Gregorini et al., 2008; Abrahamse et al., 2009; Gregorini, 2012; Pulido et al., 2015; Vibart et al., 2017). When a new paddock or strip is allocated during the afternoon, ruminants display fewer, longer and more intensive grazing bouts in late afternoon and early evening compared with daily morning allocation (Orr et al., 2001; Gregorini et al., 2006; Gregorini et al., 2008; Abrahamse et al., 2009). Although these studies have not reported that the observed shifts can increase daily herbage intake, Gregorini (2012) suggested that ruminants moved to new fresh paddocks or strips in the afternoon may have increased nutrient intake owing to longer grazing periods when herbage quality is at its peak, resulting in average increases of 5\% in daily milk yield (Orr et al., 2001; Abrahamse et al., 2009; Mattiauda et al., 2013; Pulido et al., 2015; Vibart et al., 2017).

According to Janssen (2010), the nature and amount of feed are key determinants of enteric $\mathrm{CH}_{4}$ emissions from ruminants. Therefore, diurnal variations in herbage chemical composition associated with afternoon paddock allocation could be a strategy to mitigate enteric $\mathrm{CH}_{4}$ emissions of livestock pasture-based systems. Modeling results have shown reductions in enteric $\mathrm{CH}_{4}$ emissions intensity $(\mathrm{g} / \mathrm{kg}$ of milk) by dairy cows when herbage NFC increases at the expense of fiber content (Ellis et al., 2012), and Gregorini (2012) suggested the need of field research to assess this hypothesis.

\subsection{Conceptual model, objectives and hypotheses}

Based on literature review, a conceptual model was created aiming at integrating the relationships among plant, animal and soil components as a function of grazing strategies in tropical pasture-based dairy systems (Figure 1). The objective was to assess and understand the effect of two strategies of rotational grazing management (light blue boxes; Figure 1) on plant and animal responses, and soil parameters in an intensive tropical pasture-based dairy system.

For the first phase of the study, the central hypothesis was that changing sward structure through strategies of rotational grazing management would optimize processes related to plant growth (green boxes), plant-animal interface and animal responses (yellow boxes) which would in turn, affect soil parameters that determine processes associated to $\mathrm{N}_{2} \mathrm{O}$ flux from soils (orange boxes). The objective was to describe and measure the influence of two pre-grazing targets ( $\mathrm{LI}_{95 \%}$ and $\mathrm{LI}_{\mathrm{Max}}$ ) on herbage accumulation of elephant grass (Pennisetum purpureum Schum. cv. Cameroon), milk outputs of Holstein $\times$ Jersey cows, and soil parameters $\left(\mathrm{N}_{2} \mathrm{O}\right.$ emission and WFPS, soil $\mathrm{NH}_{4}{ }^{+}$and soil $\left.\mathrm{NO}_{3}{ }^{-}\right)$. 


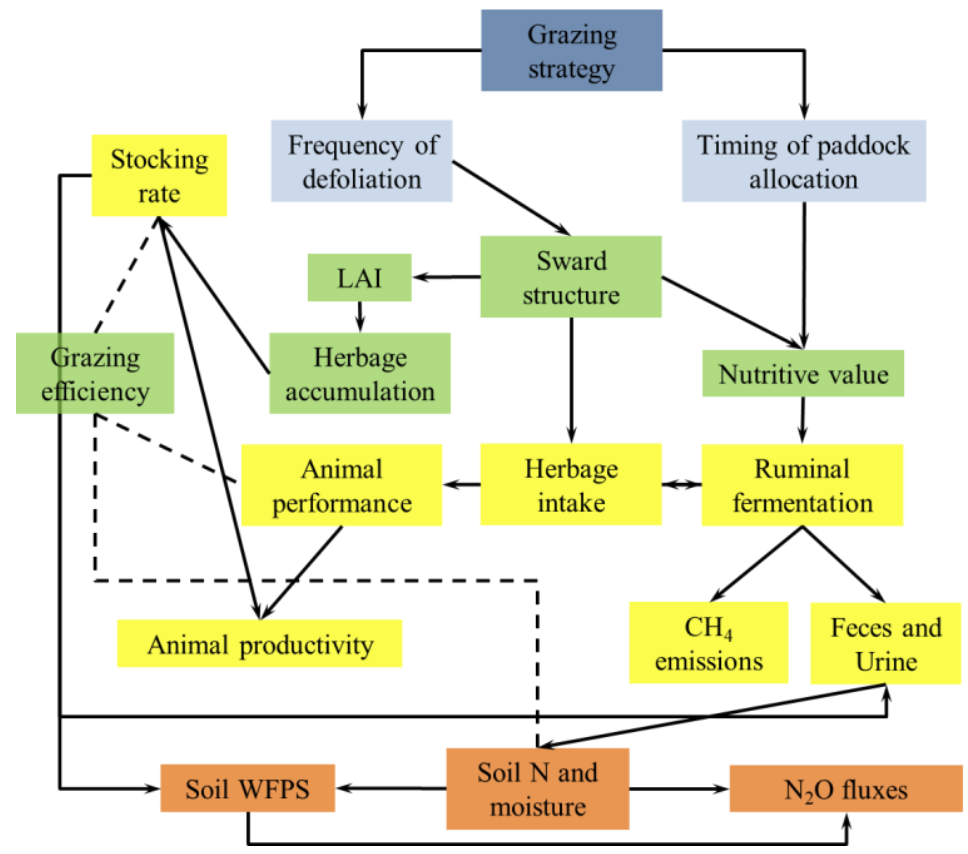

Figure 1. Conceptual model - Blue boxes: controlled factors (treatments); green boxes: plant responses; yellow boxes: animal responses; orange boxes: soil parameters

Once the ideal pre-grazing target $\left(\mathrm{LI}_{95 \%}\right.$ or $\left.\mathrm{LI}_{\mathrm{Max}}\right)$ was established during the first phase, the second step consisted of a refinement of the first phase. The hypothesis was that the diurnal variation in herbage chemical composition of elephant grass coupled with afternoon allocation of the herd to a new fresh paddock would increase nutrient intake and milk outputs, and decrease the intensity of enteric $\mathrm{CH}_{4}$ emission of Holstein $\times$ Jersey cows. The objective was to describe and measure the influence of two timings of paddock allocation (AM and PM) on elephant grass herbage chemical composition and milk outputs of Holstein $\times$ Jersey cows.

\section{References}

Abrahamse, P.A., Tamminga, S., Dijkstra, J., 2009. Effect of daily movement of dairy cattle to fresh grass in morning or afternoon on intake, grazing behaviour, rumen fermentation and milk production. J. Agr. Sci. 147:721-730. https://doi.org/10.1017/S0021859609990153.

Aguirre-Villegas, H.A., Passos-Fonseca, T.H., Reinemann, D.J., Larson, R.A., 2017. Grazing intensity affects the environmental impact of dairy systems. J. Dairy Sci. 100: 6804-6821. https://doi.org/10.3168/jds.201612325.

Allen, M.S., 1996. Physical constraints on voluntary intake of forages by ruminants. J. Anim. Sci. 74:3063-3075. https://www.ncbi.nlm.nih.gov/pubmed/8994921.

Allen, M.S., 2000. Effects of diet on short-term regulation of feed intake by lactating dairy cattle. J. Dairy Sci. 83:1598-1624. https://doi.org/10.3168/jds.S0022-0302(00)75030-2. 
Andueza, D., Delgado, I., Muñoz, F., 2012. Variation of digestibility and intake by sheep of lucerne (Medicago sativa L.) hays cut at sunrise or sunset. J. Agr. Sci. 150(2):263-270. https://doi.org/10.1017/S0021859611000542.

Barbosa, R.A., Nascimento Jr., D., Euclides, V.P.B., Da Silva, S.C., Zimmer, A.H., Torres Jr., R.A.A., 2007. Capim Tanzânia submetido a combinações entre intensidade e frequência de pastejo. Pesq. Agropec. Bras. 42:329-340. https://doi.org/10.1590/S0100-204X2007000300005.

Barbosa, R.A., Nascimento Júnior, D., Vilela, H.H., Da Silva, S.C., Euclides, V.P.B., Sbrissia, A.F., Sousa, B.M.L., 2011. Morphogenic and structural characteristics of guinea grass pastures submitted to three frequencies and two defoliation severities. Rev. Bras. Zootecn. 40 (5):947-954. http://doi.org/10.1590/S1516-35982011000500002.

Bardgett, R.D, Jones, A.C., Jones, D.L., Kemmitt, S.J., Cook, R., Hobbs, P.J., 2001. Soil microbial community patterns related to the history and intensity of grazing in sub-montane ecosystems. Soil Biol. Biochem. 33:1653-1664. https://doi.org/10.1016/S0038-0717(01)00086-4.

Bardgett, R.D., Hobbs, P.J., Frostegard, A., 1996. Changes in soil fungal:bacterial biomass ratios following reductions in the intensity of management of an upland grassland. Biol. Fertil. Soils. 22:261-264. https://doi.org/10.1007/BF00382522.

Bardgett, R.D., Wardle, D.A., 2003. Herbivore-mediated linkages between aboveground and belowground communities. Ecology. 84:2258-2268. https://doi.org/10.1890/02-0274.

Barneze, A.S., Mazzetto, A.M., Zani, C.F., Misselbrook, T., Cerri, C.C., 2014. Nitrous oxide emissions from soil due to urine deposition by grazing cattle in Brazil. Atmos. Environ. 92:394397. https://doi.org/10.1016/j.atmosenv.2014.04.046.

Barneze, A.S., Minet, E.P., Cerri, C.C., Misselbrook, T., 2015. The effect of nitrification inhibitors on nitrous oxide emissions from cattle urine deposition to grassland under summer conditions in the UK. Chemosphere. 119:122-129. https://doi.org/10.1016/j.chemosphere.2014.06.002.

Bertrand, A., Tremblay, G.F., Pelletier, S., Castonguay, Y., Bélanger, G., 2008. Yield and nutritive value of timothy as affected by temperature, photoperiod and time of harvest. Grass Forage Sci. 63:421-432. https://doi.org/10.1111/j.1365-2494.2008.00649.

Boland, T.M., Quinlan, C., Pierce, K.M., Lynch, M.B., Kelly, A.K., Purcell, P.J., 2013. The effect of pasture pre grazing vegetation mass on methane emissions, ruminal fermentation, and average daily gain of grazing beef heifers. J. Anim. Sci. 91:3867-3874. https://doi.org/10.2527/jas2013$\underline{5900}$.

Brito, A.F., Tremblay, G.F., Bertrand, A., Castonquay, Y., Bélanger, G., Michaud, R., Lafrenière, C., Martineau, R., Berthiaume, R., 2016. Performance and nitrogen use efficiency in mid-lactation dairy cows fed timothy cut in the afternoon or morning. J. Dairy Sci. 99:1-16. https://doi.org/10.3168/jds.2015-10597. 
Brito, A.F., Tremblay, G.F., Lapierre, H., Bertrand, A., Castonquay, Y., Belanger, G., Benchaar, C., Oullet, D.R., Berthiaume, R., 2008. Alfalfa cut at sundown and harvested as baleage improves milk yield of late-lactation dairy cows. J. Dairy Sci. 91:3968-3982. https://doi.org/10.3168/jds.2008-1282.

Brougham, R.W., 1955. A study in rate of pasture growth. Aust. J. Agric. Res. 6:804-812. https://doi.org/10.1071/AR9550804.

Bryant, R.H., Dalley, D.E., Gibbs, J., Edwards, G.R., 2014. Effect of grazing management on herbage protein concentration, milk production and nitrogen excretion of dairy cows in mid-lactation. Grass Forage Sci. 69:644-654. https://doi.org/10.1111/gfs.12088.

Bryant, R.H., Gregorini, P., Edwards, G.R., 2012. Effects of N fertilisation, leaf appearance and time of day on $\mathrm{N}$ fractionation and chemical composition of Lolium perenne cultivars in spring. Anim. Feed Sci. Technol. 173:210-219. https://doi.org/10.1016/j.anifeedsci.2012.02.003.

Burns, J.C., Fischer, D.S., Mayland, H.F., 2007. Diurnal shifts in nutritive value of alfalfa harvested as hay and evaluated by animal intake and digestion. Crop Sci. 47:2190-2197. https://doi.org/10.2135/cropsci2007.02.0072.

Carnevalli, R.A., Da Silva, S.C., Bueno, A.A.O., Uebele, M.C., Bueno, F.O., Hodgson, J., Silva, G.N., Morais, J.P.G., 2006. Herbage production and grazing losses in Panicum maximum cv. Mombaça under four grazing management. Trop. Grassl.-Forrajes Trop. 40:165-176. http://tropicalgrasslands.info/public/journals/4/Historic/Tropical\%20Grasslands\%20Journal\%20ar chive/PDFs/Vol 40 2006/Vol $40 \quad 03$ 2006 pp165 176.pdf.

Carvalho, P.C.F., 2013. Harry Stobbs memorial lecture: can grazing behavior support innovations in grassland management? Trop. Grassl.-Forrajes Trop. 1:137-155. https://doi.org/10.17138/TGFT(1)137-155.

Chapman, D., 2016. Using ecophysiology to improve farm efficiency: application in temperate dairy grazing systems. Agriculture 6:1-19. https://doi.org/10.3390/agriculture6020017.

Chapman, D., Lemaire, G., 1993. Morphogenetic and structural determinants of plant regrowth after defoliation. In: Proceedings of the 17th International Grassland Congress. SIR Publishing, Wellington, New Zealand, pp. 95-104.

Chatterton, N.J, Harrison, P.A, Bennett, J.H, Asay, K.H., 1989. Carbohydrate partitioning in 185 accessions of Gramineae grown under warm and cool temperatures. J. Plant Physiol. 134:169-179. https://doi.org/10.1016/S0176-1617(89)80051-3.

Chiavegato, M.B., Congio, G.F.S., Da Silva, S.C., 2018. Estratégias de manejo do pastejo para redução de impactos ambientais. In: Anais do $4^{\circ}$ Simpósio Brasileiro de Produção de Ruminantes no Cerrado: Eficiência produtiva e impacto ambiental na produção de ruminantes. UFU, Uberlândia, Brasil, $\quad$ pp. 15-36. http://www.eventos.ufu.br/sites/eventos.ufu.br/files/documentos/anais_iv_simprucerrado_versao_f inal com resumos.pdf. 
Ciavarella, T.A., Simpson, R.J., Dove, H., Leyry, B.J., Sims, I.M., 2000. Diurnal differences in the concentration of water-soluble carbohydrates in Phalaris aquatica L. pasture in spring, and the effect of short-term shading. Aust. J. Agric. Res. 51:749-756. https://doi.org/10.1071/AR99150.

Clough, T.J., Lanigan, G.J., de Klein, C.A.M., Samad, M.S., Morales, S.E., Rex, D., Bakken, L.R., Johns, C., Condron, L.M., Grant, J., Richards, K.G., 2017. Influence of soil moisture on codenitrification fluxes from a urea-affected pasture soil. Sci. Rep. 7:2185. https://doi.org/10.1038/s41598-017-02278-y.

Da Silva, S.C., Bueno, A.A.O., Carnevalli, R.A., Uebele, M.C., Bueno, F.O., Hodgson, J., Matthew, C., Arnold, J.C., Morais, J.P.G., 2009. Sward structural characteristics and herbage accumulation of Panicum maximum cv. Mombaça subject to rotational stocking managements. Sci. Agric. 66:819. https://doi.org/10.1590/S010390162009000100002.

Da Silva, S.C., Carvalho, P.C.F., 2005. Foraging behaviour and herbage intake in the favourable tropics/subtropics. In: McGilloway, D.A. (Ed.), Grassland: A Global Resource. Wageningen Academic Publishers, Wageningen, The Netherlands :pp. 81-96. http://citeseerx.ist.psu.edu/viewdoc/download?doi=10.1.1.511.4117\&rep=rep1\&type=pdf.

Da Silva, S.C., Chiavegato, M.B., Pena, K.S., Silveira, M.C.T., Barbero, L.M., Junior, S.J.S., Rodrigues, C.S., Limão, V.A., Pereira, L.E.T., 2017. Tillering dynamics of Mulato grass subjected to strategies of rotational grazing management. J. Agric. Sci. 155:1082-1092. https://doi.org/10.1017/S0021859617000223.

Da Silva, S.C., Corsi, M., 2003. Manejo do pastejo. In: Anais do $20^{\circ}$ Simpósio Sobre Manejo de Pastagens, Fealq, Piracicaba, Brasil, pp. 155-186.

Da Silva, S.C., Sbrissia, A.F., Pereira, L.E.T., 2015. Ecophysiology of C4 forage grassesunderstanding plant growth for optimising their use and management. Agriculture 5:598-625. https://doi.org/10.3390/agriculture5030598.

de Klein, C.A.M, Eckard, R.J., 2008. Targeted technologies for nitrous oxide abatement from animal agriculture. Aust. J. Exp. Agric. 48:14-20. https://doi.org/10.1071/EA07217.

de Klein, C.A.M, Luo, J., Woodward, K.B., Styles, T., Wise, B., Lindsey, S., Cox, N., 2014. The effect of nitrogen concentration in synthetic cattle urine on nitrous oxide emissions. Agric. Ecosyst. Environ. 188:85-92. https://doi.org/10.1016/j.agee.2014.02.020.

de Klein, C.A.M., Pinares-Patino, C., Waghorn, G.C., 2008. Greenhouse gas emissions. In: McDowell, R.W. (Ed.), Environmental Impacts of Pasture Based Farming. CAB International, Wallingford, Oxfordshire, UK, 1-32. https://doi.org/10.1079/9781845934118.0001.

De Oliveira, F.C.L., Sanchez, J.M.D., Vendramini, J.M.B., Lima, C.G., Luz, P.H.C., Rocha, C.O., Pereira, L.E.T., Herling, V.R., 2018. Diurnal vertical and seasonal changes in non-structural carbohydrates in Marandu palisade grass. J. Agric. Sci. 1-8. https://doi.org/10.1017/ $\underline{\text { S0021859618000394. }}$ 
De Oliveira, L.P., Paiva, A., Pereira, L.E.T., Geremia, E.V., Da Silva, S.C., 2014. Morning and afternoon sampling and herbage chemical composition of rotationally stocked elephant grass cv. Napier. Trop. Grassl.-Forrajes Trop. 2:106-107. https://doi.org/10.17138/tgft(2)106-107.

Delagarde, R., Peyraud, J.L., Delaby, L., Faverdin, P., 2000. Vertical distribution of biomass, chemical composition and pepsin-cellulase digestibility in a perennial ryegrass sward: Interaction with month of year, regrowth age and time of day. Anim. Feed Sci. Technol. 84:49-68. https://doi.org/10.1016/S0377-8401(00)00114-0.

Difante, G.S., Euclides, V.P.B., Nascimento Jr., D., Da Silva, S.C., Barbosa, R.A. \& Torres Jr., R.A.A., 2010. Desempenho e conversão alimentar de novilhos de corte em capim-tanzânia submetido a duas intensidades de pastejo sob lotação rotativa. Rev. Bras. Zootecn. 39:33-41. http://doi.org/10.1590/S1516-35982010000100005.

Difante, G.S., Euclides, V.P.B., Nascimento Júnior, D., Da Silva, S.C., Torres Júnior, R.A.A., Sarmento, D.O.L., 2009a. Ingestive behaviour, herbage intake and grazing efficiency of beef cattle steers on Tanzania guineagrass subjected to rotational stocking managements. Rev. Bras. Zootecn. 38 (6):1001-1008. http://doi.org/10.1590/S1516-35982009000600005.

Difante, G.S., Nascimento Júnior, D., Euclides, V.P.B., Da Silva, S.C., Barbosa, R.A., Gonçalves, W.V., 2009b. Sward structure and nutritive value of Tanzânia guineagrass subject to rotational stocking managements. Rev. Bras. Zootecn. 38 (1):9-19. http://doi.org/10.1590/S1516$\underline{35982009000100002 .}$

Ellis, J.L., Dijkstra, J., France, J., Parsons, A.J., Edwards, G.R., Rasmussen, S., Kebreab, E., Bannink, A., 2012. Effect of high-sugar grasses on methane emissions simulated using a dynamic model. J. Dairy Sci. 95:272-285. https://doi.org/10.3168/jds.2011-4385.

Euclides, V.P.B., Carpejani, G.C., Montagner, D.B., Nascimento Júnior, D., Barbosa, R.A., Difante, G.S., 2018. Maintaining post-grazing sward height of Panicum maximum (cv. Mombacça) at 50 $\mathrm{cm}$ led to higher animal performance compared with post-grazing height of $30 \mathrm{~cm}$. Grass Forage Sci. 73:174-182. http://doi.org/10.1111/gfs.12292.

Euclides, V.P.B., Lopes, F.C., Nascimento Júnior, D., Da Silva, S.C., Difante, G.D., Barbosa, R.A. 2015. Steer performance on Panicum maximum (cv. Mombacça) pastures under two grazing intensities. Anim. Prod. Sci. 11:1849-1856. http://doi.org/10.1071/AN14721.

Euclides, V.P.B., Montagner, D.B., Difante, G.S., Barbosa, R.A., Fernandes, W.S. 2014. Sward structure and livestock performance in guinea grass cv. Tanzania pastures managed by rotational stocking strategies. Sci. Agric. 71:451-457. http://doi.org/10.1590/0103-9016-2013-0272.

Fisher, D.S., Mayland, H.F., Burns, J.C., 2002. Variation in ruminant preference for alfalfa hays cut at either sundown or sunup. Crop Sci. 42:231-237. https://doi.org/10.2135/cropsci2002.2310. 
Flechard, C.R., Ambus, P., Skiba, U., Rees, R.M., Hensen, A., van Amstel, A., Pol-van Dasselaar, A.V., Soussana, J.F., Jones, M., Clifton-Brown, J., Raschi, A., Horvath, L., Neftel, A., Jocher, M., Ammann, C., Leifeld, J., Fuhrer, J., Calanca, P., Thalman, E., Pilegaard, K., Di Marco, C., Campbell, C., Nemitz, E., Hargreaves, K.J., Levy, P.E., Ball, B.C., Jones, S.K., van de Bulk, W.C.M., Groot, T., Blom, M., Domingues, R., Kasper, G., Allard, V., Ceschia, E., Cellier, P., Laville, P., Henault, C., Bizouard, F., Abdalla, M., Williams, M., Baronti, S., Berretti, F., Grosz, B., 2007. Effects of climate and management intensity on nitrous oxide emissions in grassland systems across Europe. Agric. Ecosyst. Environ. 121:135-152. https://doi.org/10.1016/j.agee.2006.12.024.

Fonseca, L., Carvalho, P.C.F., Mezzalira, J.C., Bremm, C., Galli, J.R., Gregorini, P., 2013. Effect of sward surface height and level of herbage depletion on bite features of cattle grazing Sorghum bicolor swards. J. Anim. Sci. 91:4357-4365. https://doi.org/10.2527/jas.2012-5602.

Fonseca, L., Mezzalira, J.C., Bremm, C., Filho, R.S.A., Gonda, H.L., Carvalho, P.C.F., 2012. Management targets formaximising the short-term herbage intake rate of cattle grazing in Sorghum bicolor. Livest. Sci. 145:205-211. https://doi.org/10.1016/j.livsci.2012.02.003.

Forbes, J., 2007. Voluntary Food Intake and Diet Selection in Farm Animals. 2nd ed. CAB International, Wallingford, UK 453p. https://doi.org/10.1079/9781845932794.0000.

Gardiner, C.A., Clough, T.J., Cameron, K.C., Di, H.J., Edwards, G.R., de Klein, C.A.M., 2016. Potential for forage diet manipulation in New Zealand pasture ecosystems to mitigate ruminant urine derived N2O emissions: a review. N. Z. J. Agric. Res. 59(3):301-317. https://doi.org/10.1080/00288233.2016.1190386.

Gardiner, C.A., Clough, T.J., Cameron, K.C., Di, H.J., Edwards, G.R., de Klein, C.A.M., 2016. Potential inhibition of urine patch nitrous oxide emissions by Plantago lanceolata and its metabolite aucubin. N. Z. J. Agric. Res. 60(4):1-9. https://doi.org/10.1080/00288233.2017.1411953.

Gastal, F., Durand, J.L., 2000. Effects of nitrogen and water supply on N and C fluxes and partitioning in defoliated swards. In: Lemaire, G., Hodgson, J., de Moraes, A., Nabinger, C., de Faccio Carvalho, P.C. (Eds.), Grassland Ecophysiology and Grazing Ecology. CAB International, Wallingford, UK :pp. 15-29. https://www.cabi.org/cabebooks/ebook/20003019241.

Geremia, E.V., Pereira, L.E.T., Paiva, A.J., Oliveira, L.P., Da Silva, S.C., 2014. Intake rate and nutritive value of elephant grass cv. Napier subjected to strategies of rotational stocking management. Trop. Grass1.-Forrajes Trop. 2:51-52. https://doi.org/10.17138/tgft(2)51-52.

Giacomini, A.A., Da Silva, S.C., Sarmento, D.O.L., Zeferino, C.V., Souza Jr., S.J., Trindade, J.K., Guarda, V.D., Nascimento Jr., D., 2009. Growth of marandu palisadegrass subjected to strategies of intermittent stocking. Sci. Agric. 66:733-741. http://doi.org/10.1590/S010390162009000600003. 
Gibb, M.J., 2007. Grassland management with emphasis on grazing behaviour. In: Fresh herbage for dairy cattle. Eds A Elgersma, J Dijkstra, S Tamminga. pp. 141-157. https://library.wur.nl/ojs/index.php/frontis/article/view/1250.

Gibb, M.J., Huckle, C.A., Nuthall, R., 1998. Effect of time of day on grazing behaviour by lactating dairy cows. Grass Forage Sci. 53:41-46. https://doi.org/10.1046/j.1365-2494.1998.00102.

Gimenes, F.M.A., Da Silva, S.C., Fialho, C.A., Gomes, M.B., Berndt, A., Gerdes, L., Colozza, M.T., 2011. Ganho de peso e produtividade animal em capim-marandu sob pastejo rotativo e adubação nitrogenada. Pesq. Agropec. Bras. 46:751-759. https://doi.org/10.1590/S0100204X2011000700011.

Gregorini, P. 2012. Diurnal grazing pattern: its physiological basis and strategic management. Anim. Prod. Sci. 52:416-430. http://dx.doi.org/10.1071/AN11250.

Gregorini, P., Beukes, P.C., Bryant, R.H., Romera, A.J., 2010. A brief overview and simulation of the effects of some feeding strategies on nitrogen excretion and enteric methane emission from grazing dairy cows. In: Edwards G.R. and Bryant R.H. (eds) Proceedings of the 4th Australasian Dairy Science Symposium. Canterbury, New Zealand: Lincoln University. http://www.sciquest.org.nz/elibrary/download/69329/A+brief+overview+and+simulation+of+the+ effects+of+some+feeding+strategies+on+nitrogen+excretion+and+enteric+methane+emission+fro $\underline{m+g r a z i n g}+$ dairy + cows.

Gregorini, P., Beukes, P.C., Dalley, D., Romera, A.J., 2016. Screening for diets that reduce urinary nitrogen excretion and methane emissions while maintaining or increasing production by dairy cows. Sci. Total Environ. 551-552:32-41. https://doi.org/10.1016/j.scitotenv.2016.01.203.

Gregorini, P., Eirin, M., Refi, R., Ursino, M., Ansin, O.E., Gunter, S.A., 2006. Timing of herbage allocation: Effect on beef heifers daily grazing pattern and performance. J. Anim. Sci. 84:19431950. https://doi.org/10.2527/jas.2005-537.

Gregorini, P., Gunter, S.A., Beck, P.A., 2008. Matching plant and animal processes to alter nutrient supply in strip grazed cattle: timing of herbage and fasting allocation. J. Anim. Sci. 86:1006-1020. https://doi.org/10.2527/jas.2007-0432.

Gregorini, P., Gunter, S.A., Masino, C.A., Beck, P.A., 2007. Effects of ruminal fill on intake rate and grazing dynamics of beef heifers. Grass Forage Sci. 62:346-354. https://doi.org/10.1111/j.13652494.2007.00589.

Gregorini, P., Soder, K.J., Sanderson, M.A., Ziegler, G., 2009. Toughness, particle size and chemical composition of meadow fescue (Festuca pratensis Hud.) herbage as affected by time of day. Anim. Feed Sci. Technol. 151:330-336. https://doi.org/10.1016/j.anifeedsci.2009.02.004.

Griggs, T.C., MacAdam, J.W., Mayland, H.F., Burns, J.C., 2005. Nonstructural carbohydrate and digestibility patterns in orchardgrass swards during daily defoliation sequences initiated in evening and morning. Crop Sci. 45:1295-1304. https://doi.org/10.2135/cropsci2003.0613. 
Guerci, M., Knudsen, M.T., Bava, L., Zucali, M., Schonbach, P., Kristensen, T., 2013. Parameters affecting the environmental impact of a range of dairy farming systems in Denmark, Germany and Italy. J. Clean. Prod. 54:133-141. https://doi.org/10.1016/j.jclepro.2013.04.035.

Janssen, P.H., 2010. Influence of hydrogen on rumen methane formation and fermentation balances through microbial growth kinetics and fermentation thermodynamics. Anim. Feed Sci. Technol. 160:1-22. https://doi.org/10.1016/j.anifeedsci.2010.07.002.

Laca, E.A., Lemaire, G., 2000. Measuring sward structure. In: Mannetje, L., Jones, R.M. (Eds.), Field and Laboratory Methods for Grassland and Animal Production Research. CABI, Wallingford, UK, pp. 103-121 https://doi.org/10.1079/9780851993515.0000.

Lechtenberg, V.L., Holt, D.A., Youngberg, H.W., 1971. Diurnal variation in nonstructural carbohydrates, in vitro digestibility, and leaf to stem ratio of alfalfa. Agron. J. 63:719-724. https://doi.org/10.2134/agronj1971.00021962006300050019x.

Lessa, A.C.R., Madari, B.E., Paredes, D.S., Boddey, R.M., Urquiaga, S., Jantalia, C.P., Alves, B.J.R., 2014. Bovine urine and dung deposited on Brazilian savannah pastures contribute differently to direct and indirect soil nitrous oxide emissions. Agr. Ecosyst. Environ. 190:104-111. https://doi.org/10.1016/j.agee.2014.01.010.

Levine, U.Y., Teal, T.K., Robertson, G.P., Schmidt, T.M., 2011. Agriculture's impact on microbial diversity and associated fluxes of carbon dioxide and methane. ISME J. 5:1683-1691. https://doi.org/10.1038/ismej.2011.40.

Lu, Y., Gehan, J.P., Sharkey, T.D., 2005. Daylength and circadian effects on starch degradation and maltose metabolism. Plant Physiol. 38:2280-2291. https://doi.org/10.1104/pp.105.061903.

Luo, J., Wyatt, J., van der Weerden, T., Thomas, S., de Klein, C., Li, Y., Rollo, M., Lindsey, S., Ledgard, S., Li, J., Ding, W., Qin, S., Zhang, N., Bolan, N., Kirkham, M.B., Bai, Z., Ma, L., Zhang, X., Wang, H., Liu, H., Rys, G., 2017. Potential hotspot areas of nitrous oxide emissions from grazed pastoral dairy farm systems. Adv. Agron. 145:205-268. https://doi.org/10.1016/bs.agron.2017.05.006.

Luo, J., Balvert, S.F., Wise, B., Welten, B., Ledgard, S.F., de Klein, C.A.M., Lindsey, S., Judge, A., 2018. Using alternative forage species to reduce emissions of the greenhouse gas nitrous oxide from cattle urine deposited onto soil. Sci. Total Environ. 610-611:1271-1280. https://doi.org/10.1016/j.scitotenv.2017.08.186.

Mattiauda, D.A., Tamminga, S., Gibb, M.J., Soca, P., Bentancur, O., Chilibroste, P., 2013. Restricting access time at pasture and time of grazing allocation for Holstein dairy cows: Ingestive behaviour, dry matter intake and milk production. Livest. Sci. 152:53-62. https://doi.org/10.1016/j.livsci.2012.12.010.

Mayland, H.F., Shewmaker, G.E., Harrison, P.A., Chatterton, N.J., 2000. Nonstructural carbohydrates in tall fescue cultivars: Relationship to animal preference. Agron. J. 92:1203-1206. https://doi.org/10.2134/agronj2000.9261203x. 
Mazzetto, A.M., Barneze, A.S., Feigl, B.J., van Groenigen, J.W., Oenema, O., Cerri, C.C., 2014. Temperature and moisture affect methane and nitrous oxide emission from bovine manure patches in tropical conditions. Soil Biol Biochem 76:242-248. https://doi.org/10.1016/j.soilbio.2014.05.026.

Mazzetto, A.M., Barneze, A.S., Feigle, B.J., van Groenigen, J.W., Oenema, O., de Klein, C.A.M., Cerri, C.C., 2015. Use of the nitrification inhibitor dicyandiamide (DCD) does not mitigate N2O emission from bovine urine patches under Oxisol in Northwest Brazil. Nutr. Cycl. Agroecosyst. 101:83-92. https://doi.org/10.1007/s10705-014-9663-4.

Mertens, D.R., 1994. Regulation of forage intake. In: Fahey Jr., G.C., Collins, M., Mertens, D.R., Moser, L.E. (Eds.), Forage Quality, Evaluation, and Utilization. American Society of Agronomy, Crop Science Society of America, Soil Science Society of America, Madison, WI, pp. 450-493. https://dl.sciencesocieties.org/publications/.../foragequalityev/45.

Mezzalira, J.C., Carvalho, P.C.F., Fonseca, L. Bremm, C., Cangiano, C., Gonda, H.L., Laca, E.A., 2014. Behavioural mechanisms of intake rate by heifers grazing swards of contrasting structures. Appl. Anim. Behav. Sci. 153:1-9. https://doi.org/10.1016/j.applanim.2013.12.014.

Moore, J.E., 1994. Forage quality indices: development and application. In: Fahey Jr., G.C. (Ed.), Forage Quality, Evaluation and Utilization. ASA, CSSA/SSSA, Madison, WI, pp. 967-998.

Morin, C., Bélanger, G., Tremblay, G.F., Bertrand, A., Castonguay, Y., Drapeau, R., Michaud, R., Berthiaume, R., Allard, G., 2011. Diurnal variations of nonstructural carbohydrates and nutritive value in alfalfa. Crop Sci. 51:1297-1306. https://doi.org/10.2135/cropsci2010.07.0406.

Morin, C., Bélanger, G., Tremblay, G.F., Bertrand, A., Castonguay, Y., Drapeau, R., Michaud, R., Berthiaume, R., Allard, G., 2012. Diurnal variations of nonstructural carbohydrates and nutritive value in timothy. Can. J. Plant Sci. 92:883-887. https://doi.org/10.1139/CJPS2011-272.

Mott, G.O., 1960. Grazing pressure and measurement of pasture production. In: Proceedings of the 8th International Grassland Congress, Reading, UK, pp. 606-611.

Muñoz, C., Letelier, P.A., Ungerfeld, E.M., Morales, J.M., Hube, S., Pérez-Prieto, L.A., 2016. Effects of pre grazing herbage mass in late spring on enteric methane emissions, dry matter intake, and milk production of dairy cows. J. Dairy Sci. 99:7945-7955. https://doi.org/10.3168/jds.2016$\underline{10919 .}$.

O’Brien, D., Shalloo, L., Patton, J., Buckley, F., Grainger, C., Wallace, M., 2012. A life cycle assessment of seasonal grass-based and confinement dairy farms. Agr. Syst. 107:33-46. https://doi.org/10.1016/j.agsy.2011.11.004.

Orr, R.J., Penning, P.D., Harvey, A., Champion, R.A., 1997. Diurnal patterns of intake rate by sheep grazing monocultures of rye grass or white clover. Appl. Anim. Behav. Sci. 53:65-77. https://doi.org/10.1016/S0168-1591(96)01120-3.

Orr, R.J., Rutter, S.M., Penning, P.D., Rook, A.J., 2001. Matching grass supply to grazing patterns for dairy cows. Grass Forage Sci. 56:352-361. https://doi.org/10.1046/j.1365-2494.2001.00284.x. 
Palhano, A.L., Carvalho, P.C.F., Dittrich, J.R., Moraes, A., Da Silva, S.C.,Monteiro, A.L.G., 2007. Características do processo de ingestão de forragem por novilhas holandesas em pastagens de capim-mombaça. Rev. Bras. Zootecn. 36:1014-1021. https://doi.org/10.1590/S1516$\underline{35982007000500005 .}$.

Parsons, A.J, Leafe, E.L., Collet, B., Penning, P.D., Lewis, J., 1983. The physiology of grass production under grazing. II. Photosynthesis, crop growth and animal intake of continuouslygrazed swards. J. Appl. Ecol. 20:127-139. https://doi.org/10.2307/2403381.

Pedreira, B.C., Pedreira, C.G.S., Da Silva, S.C., 2009. Acúmulo de forragem durante a rebrotação de capim-xaraés submetido a três estratégias de desfolhação. Rev. Bras. Zootecn. 38:618-625. http://doi.org/10.1590/S1516-35982009000400005.

Pedreira, C.G.S., Braga, G.J., Portela, J.N. 2017. Herbage accumulation, plant-part composition and nutritive value on grazed signal grass (Brachiaria decumbens) pastures in response to stubble height and rest period based on canopy light interception. Crop Pasture Sci. 68:62-73. http://doi.org/10.1071/CP16333.

Pelletier, S., Tremblay, G.F., Belanger, G., Bertrand, A., Castonguay, Y., Pageau, D., Drapeau, R., 2010. Forage nonstructural carbohydrates and nutritive value as affected by time of cutting and species. Agron. J. 105:1388-1398. https://doi.org/10.2134/agronj2010.0158.

Pereira, L.E.T., Paiva, A.J., Geremia, E.V., Da Silva, S.C., 2014. Components of herbage accumulation in elephant grass cvar Napier subjected to strategies of intermittent stocking management. J. Agric. Sci. 152:954-966. https://doi.org/10.1017/S0021859613000695.

Pereira, L.E.T., Paiva, A.J., Geremia, E.V., da Silva, S.C., 2015a. Grazing management and tussock distribution in elephant grass. Grass Forage Sci. 70:1-12. https://doi.org/10.1111/gfs.12137.

Pereira, L.E.T., Paiva, A.J., Geremia, E.V., Da Silva, S.C., 2015b. Regrowth patterns of elephant grass (Pennisetum purpureum Schum.) subjected to strategies of intermittent stocking management. Grass Forage Sci. 70:195-204. https://doi.org/10.1111/gfs.12103.

Pereira, L.E.T., Paiva, A.J., Geremia, E.V., Da Silva, S.C., 2018. Contribution of basal and aerial tillers to sward growth in intermittently stocked elephant grass. Grassl. Sci. 64:108-117. https://doi.org./10.1111/grs.12194.

Perry, L.J., Jr., Moser, L.E., 1974. Carbohydrate and organic nitrogen concentrations within range grass parts at maturity. J. Range Manage. 27:276-278.

Pollock, C.J., Cairns, A.J., 1991. Fructan metabolism in grasses and cereals. Annu. Rev. Plant Physiol. Plant Mol. Biol. 42:77-101. https://doi-org.ez67/10.1146/annurev.pp.42.060191.000453.

Poppi, D.P., Hughes, T.P., L'Huillier, P.J., 1987. Intake of pasture by grazing ruminants. In: Nicol, A.M. (Ed.), Feeding Livestock on Pasture. Occasional Publication, New Zealand Society of Animal Production, Hamilton, New Zealand, pp. 55-63. 
Pulido, R.G., Ruiz-Albarran, M. Balocchi, O.A., Nannig, P., Wittwer, F., 2015. Effect of timing of pasture allocation on production, behavior, rumen function, and metabolism of early lactating dairy cows during autumn. Livest. Sci. 177:43-51. https://doi.org/10.1016/j.livsci.2015.04.002.

Rafique, R., Hennessy, D., Kiely, G., 2011. Nitrous oxide emission from grazed grassland under different management systems. Ecosystems. 14:563-582. https://doi.org/10.1007/s10021-0119434-x.

Rex, D., Clough, T.J., Richards, K.G., de Klein, C.A.M., Morales, S.E., Samad, M.S., Grant, J., Lanigan, G.J., 2018. Fungal and bacterial contributions to codenitrification emissions of N2O and N2 following urea deposition to soil. Nutr. Cycl. Agroecosyst. 110(1):135-149. https://doi.org/10.1007/s10705-017-9901-7.

Saggar, S., Jha, N., Deslippe, J., Bolan, N.S., Luo, J., Giltrap, D.L., Kim, D.G., Zaman, M., Tillman, R.W., 2013. Denitrification and N2O:N2 production in temperate grasslands: process, measurements, modelling and mitigating negative impacts. Sci. Total Environ. 465:173-195. https://doi.org/10.1016/j.scitotenv.2012.11.050.

Samad, M.D.S., Biswas, A., Bakken, L.R., Clough, T.J., de Klein, C.A.M., Richards, K.G., Lanigan, G.J., Morales, S.E., 2016. Phylogenetic and functional potential links pH and N2O emissions in pasture soils. Sci. Rep. 6:35990. https://doi.org/10.1038/srep35990.

Santos, F.A.P., Dorea, J.R.R., de Souza, J., Batistel, F., Costa, D.F.A., 2014. Forage management and methods to improve nutrient intake in grazing cattle. In: Proceedings of the 25th Annual Florida Ruminant Nutrition Symposium. University of Florida, Gainesville, United States of America, pp. 144-164. http://dairy.ifas.ufl.edu/rns/2014/santos.pdf.

Sbrissia, A.F., Duchini, P.G., Zanini, G.D., Santos, G.T., Padilha, D.A., Schimitt, D., 2018. Defoliation strategies in pastures submitted to intermittent stocking method: underlying mechanisms buffering forage accumulation over a range of grazing heights. Crop Sci. 58:1-10. https://doi.org./10.2135/cropsci2017.07.0447.

Schmalz, H.J., Taylor, R.V., Johnson, T.N., Kennedy, P.L., DeBano, S.J., Newingham, B.A., McDaniel, P.A., 2013. Soil Morphologic properties and cattle stocking rate affect dynamic soil properties. Rangeland Ecol. Manag. 66(4):445-453. https://doi.org/10.2111/REM-D-12-00040.1.

Shewmaker, G.E., Mayland, H.F., Roberts, C.A., Harrison, P.A., Chatterton, N.J., Sleper, D.A., 2006. Daily carbohydrate accumulation in eight tall fescue cultivars. Grass Forage Sci. 61:413-421. https://doi.org/10.1111/j.1365-2494.2006.00550.x.

Silva, A.P., Imhoff, S., Corsi, M., 2003. Evaluation of soil compaction in irrigated short-duration grazing system. Soil Tillage Res. 70:83-90. https://doi.org/10.1016/S0167-1987(02)00122-8.

Silveira, M.C.T., Da Silva, S.C., Souza Jr., S.J., Barbero, L.M., Rodrigues, C.S., Limão, V.A., Pena, K.S., Nascimento Jr., D., 2013. Herbage accumulation and grazing losses on Mulato grass subjected to strategies of rotational stocking management. Sci. Agric. 70:242-249. https://doi.org/10.1590/S0103-90162013000400004. 
Silveira, M.C.T., Nascimento Jr., D., Rodrigues, C.S., Pena, K.S., Souza Jr., S.J., Barbero, L.M., Limão, V.A., Euclides, V.P.B., Da Silva, S.C., 2016. Forage sward structure of Mulato grass (Brachiaria hybrid ssp.) subjected to rotational stocking strategies. Aust. J. Crop Sci. 10(6):864873. https://doi.org/10.21475/ajcs.2016.10.06.p7568.

Smith, P., Goulding, K.W., Smith, K.A., Powlson, D.S., Smith, J.U., Falloon, P., Coleman, K., 2001. Enhancing the carbon sink in European agricultural soils: Including trace gas fluxes in estimates of carbon mitigation potential. Nutr. Cycl. Agroecosyst. 60:237-252. https://doi.org/10.1023/A:1012617517839.

Trevaskis, L.M., Fulkerson, W.J., Gooden, M., 2001. Provision of certain carbohydrate-based supplements to pasture-fed sheep, as well as time of harvesting of the pasture, influences $\mathrm{pH}$, ammonia concentration and microbial protein synthesis in the rumen. Aust. J. Exp. Agric. 41:2127. https://doi.org/10.1071/EA00063.

Trindade, J.K., Da Silva, S.C., Souza Jr, S.J., Giacomini, A.A., Zeferino, C.V., Guarda, V.D.A., Carvalho, P.C.F., 2007. Composição morfológica da forragem consumida por bovinos de corte durante o rebaixamento do capim-marandu submetido a estratégias de pastejo rotativo. Pesq. Agropec. Bras. 42:883-890. https://doi.org/10.1590/S0100204X2007000600016.

van der Weerden, T.J., Styles, T.M., Rutherford, A.J., de Klein, C.A.M., Dynes, R., 2017. Nitrous oxide emissions from cattle urine deposited onto soil supporting a winter forage kale crop. N. Z. J. Agric. Res. 60:119-130. https://doi.org/10.1080/00288233.2016.1273838.

Vasta, V., Pagano, R,I., Luciano, G., Scerra, M.,Caparra, P., Foti, F., Cilione, C., Biondi, L., Priolo, A., Avondo, M., 2012. Effect of morning vs. afternoon grazing on intramuscular fatty acid composition in lamb. Meat Sci. 90:93-98. http://dx.doi.org/10.1016/j.meatsci.2011.06.009.

Venterea, R., Clough, T.J., Coulter, J.A., Breuillin-Sessoms, F., Wang, P., Sadowsky, M.J., 2015. Ammonium sorption and ammonia inhibition of nitrite-oxidizing bacteria explain contrasting soil N2O production. Sci. Rep. 5:12153. https://doi.org/10.1038/srep12153.

Vibart, R.E., Tavendale, M., Otter, D., Schwendel, B.H., Lowe, K., Gregorini, P., Pacheco, D., 2017. Milk production and composition, nitrogen utilization, and grazing behavior of late-lactation dairy cows as affected by time of allocation of a fresh strip of pasture. J. Dairy Sci. 100:1-14. http://dx.doi.org/10.3168/jds.2016-12413.

Voltolini, T.V., Santos, F.A.P., Martinez, J.C., Clarindo, R.L., Penati, M.A., Imaizumi, H., 2010a. Características produtivas e qualitativas do capim-elefante pastejado em intervalo fixo ou variável de acordo com a interceptação da radiação fotossinteticamente ativa. Rev. Bras. Zootec. 39 (5):1002-1010. http://doi.org/10.1590/S1516-35982010000500009.

Voltolini, T.V., Santos, F.A.P., Martinez, J.C., Imaizumi, H., Clarindo, R.L., Penati, M.A., 2010b. Produção e composição do leite de vacas mantidas em pastagens de capim-elefante submetidas a duas frequências de pastejo. Rev. Bras. Zootecn. 39 (1):121-127. https://doi.org/10.1590/S1516$\underline{35982010000100016 .}$ 
Wade, M.H., Carvalho, P.C.F., 2000. Defoliation patterns and herbage intake in grazed pastures. In: Lemaire, G., Hodgson, J., de Moraes, A., Carvalho, P.D.F., Nabinger, C. (Eds.), Ecophysiology of Grasslands and the Ecology of Grazing. CAB Int., Oxford, UK: pp. 233-248 https://doi.org/10.1079/9780851994529.0233.

Warren, S.D., Thurow, T.L., Blackburn, W.H., Garza, N.E., 1986. The influence of livestock trampling under intensive rotation grazing on soil hydrologic characteristics. J. Range Manage. 39:491-495. https://doi.org/10.2307/3898755.

Weise, S. E., Wijk, K.J.V., Sharkey, T.D., 2011. The role of transitory starch in $\mathrm{C}_{3}$, CAM e $\mathrm{C}_{4}$ metabolism and opportunities for engineering leaf starch accumulation. J. Exp. Bot. 62:31093118. https://doi.org/10.1093/jxb/err035.

White, L.M., 1973. Carbohydrate reserves of grasses: a review. J. Range Manage. 26: 13-18. https://doi.org/10.2307/3896873.

Wilson, J.R., Kennedy, P.M., 1996. Plant and animal constraints to voluntary feed intake associated with fibre characteristics and particle breakdown and passage in ruminants. Aust. J. Agric. Res. 47:199-225. https://doi.org/10.1071/AR9960199.

Wims, C.M., Deighton, M.H., Lewis, E., O'Loughlin, B., Delaby, L., Boland, T.M., O'Donovan, M., 2010. Effect of pregrazing herbage mass on methane production, dry matter intake, and milk production of grazing dairy cows during the mid-season period. J. Dairy Sci. 93:4976-4985. https://doi.org/10.3168/jds.2010-3245.

Wrage, N., Velthof, G.L., van Beusichem, M.L., Oenema, O., 2001. Role of nitrifier denitrification in the production of nitrous oxide. Soil Biol. Biochem. 33:1723-1732. https://doi.org/10.1016/S0038-0717(01)00096-7.

Zanini, G.D., Santos, G.T., Schmitt, D., Padilha, D.S., Sbrissia, A.F., 2012. Distribuição de colmo na estrutura vertical de pastos de capim Aruana e azevém anual submetidos a pastejo intermitente por ovinos. Cienc. Rural. 42 (5):882-887. http://doi.org/10.1590/S0103-84782012000500020.

Zeeman, S.C., Smith, S.M., Smith, A.M., 2007. Review article: The diurnal metabolism of leaf starch. Biochem. J. 401:13-28. https://doi.org/10.1042/BJ20061393. 


\title{
3. STRATEGIC GRAZING MANAGEMENT TOWARDS SUSTAINABLE INTENSIFICATION AT TROPICAL PASTURE-BASED DAIRY SYSTEMS ${ }^{1}$
}

\begin{abstract}
Agricultural systems are responsible for environmental impacts that can be mitigated through the adoption of more sustainable practices. The objective of this study was to investigate the influence of two pre-grazing targets (95\% and maximum canopy light interception during pasture regrowth; $\mathrm{LI}_{95 \%}$ and $\mathrm{LI}_{\text {Max }}$, respectively) on sward structure and herbage nutritive value of rotationally grazed elephant grass (Pennisetum purpureum Schum. cv. Cameroon), and dry matter intake (DMI), milk yield, stocking rate, enteric methane $\left(\mathrm{CH}_{4}\right)$ emissions by Holstein $\times$ Jersey dairy cows. It was hypothesized that grazing strategies can modify sward structure and improve the nutritive value of the consumed herbage, increasing DMI and reducing the intensity of enteric $\mathrm{CH}_{4}$ emissions, providing environmental and productivity benefits to tropical pasture-based dairy systems. Results indicated that pre-grazing sward surface height was greater for $\mathrm{LI}_{\mathrm{Max}}(\approx 135 \mathrm{~cm})$ than $\mathrm{LI}_{95 \%}(\approx 100 \mathrm{~cm})$ and can be used as a reliable field guide for monitoring sward structure. Grazing management based on $\mathrm{LI}_{95 \%}$ criterion improved herbage nutritive value and grazing efficiency, allowing greater DMI, milk yield and stocking rate by dairy cows. Daily enteric $\mathrm{CH}_{4}$ emission was not affected; however, cows grazing elephant grass at $\mathrm{LI}_{95 \%}$ were more efficient and emitted $21 \%$ less $\mathrm{CH}_{4} / \mathrm{kg}$ of milk yield and $18 \%$ less $\mathrm{CH}_{4} / \mathrm{kg}$ of DMI. The $51 \%$ increase in milk yield per hectare overcame the $29 \%$ increase in enteric $\mathrm{CH}_{4}$ emissions per hectare in $\mathrm{LI}_{95 \%}$ grazing management. Thereby the same resource allocation resulted in a $16 \%$ mitigation of the main greenhouse gas from pasture-based dairy systems. Overall, strategic grazing management is an environmentally friendly practice that improves the use efficiency of allocated resources through optimization of processes involving plant, ruminant and their interface, and enhances milk production efficiency of tropical pasture-based systems.
\end{abstract}

Keywords: Canopy light interception; Enteric methane emissions; Herbage quality; Land-use improvement; Milk production efficiency; Elephant grass

\subsection{Introduction}

To meet the world's future food demand and environmental needs, agricultural outputs must grow from 60 to 120\% (Godfray et al., 2010; Conforti, 2011; Alexandratos and Bruinsma, 2012) while agriculture environmental footprint must decrease dramatically (Foley et al., 2011). In developing countries, agriculture production must increase $80 \%$ through higher yields resulting from intensification of existing agricultural systems (Conforti, 2011). Sustainable intensification was defined as a form of production wherein yields are increased without adverse environmental impact and without the cultivation of more land (Royal Society, 2009). Despite contested (Struik and Kuyper, 2017), this term was deeply discussed (Pretty and Bharucha, 2014) and highlights the needs to increase the productivity (i.e. agricultural product outputs per hectare) of current agricultural systems through practices that minimize key environmental issues (Garnett and Godfray, 2012).

${ }^{1}$ Congio, G.F.S., Batalha, C.D.A., Chiavegato, M.B., Berndt, A., Oliveira, P.P.A., Frighetto, R.T.S., Maxwell, T.M.R., Gregorini, P., Da Silva, S.C., 2018. Strategic grazing management towards sustainable intensification at tropical pasture-based dairy systems. Sci. Total Environ. 636:872-880. DOI: 10.1016/j.scitotenv.2018.04.301 
Intensification of pasture-based dairy systems has been associated with increasing inputs such as nitrogen fertilizer or imported supplements (Beukes et al., 2012; Foote et al., 2015; Macdonald et al., 2017). However, such intensification practices are associated with issues of environmental concern, namely increased greenhouse gases (GHG) emissions, water and land degradation (Foley et al., 2011; Vogeler et al., 2013; Foote et al., 2015). Alternatively, grazing management strategies that optimize herbage utilization and digestible dry matter intake (DMI) by grazing cows could improve land-use and mitigate key environmental issues of pasture-based dairy systems (Muñoz et al., 2016; Gregorini et al., 2017).

Plant growth is a function of canopy light interception (LI) and leaf area index (LAI), with the accumulation of herbage fitted to a sigmoid curve with three distinct phases (Brougham, 1955). During the early stages of regrowth, leaves are the morphological component accumulated the most. As LAI increases, canopy light intra-competition increases and plants change their growth pattern as a means of optimizing light capture through stem elongation. The shift in growth pattern occurs when canopy LI reaches and exceeds 95\% ( $\mathrm{LI}_{95 \%}$; Da Silva et al., 2015). Intermittent grazing practices (i.e. rotational stocking), interrupting regrowth at $\mathrm{LI}_{95 \%}$, leads to a greater leaf accumulation (Pereira et al., 2014; Pereira et al., 2015b), higher tiller population density and soil cover (Pereira et al., 2015a) than grazing at maximum light interception ( $\mathrm{LI}_{\mathrm{Max}}$ ). In addition, sward grazed at $\mathrm{LI}_{95 \%}$ has been reported to have herbage of greater nutritive value (Trindade et al., 2007) and less herbage losses (Silveira et al., 2013).

Considering the grazing animal, pre-grazing management targets which optimize leaf production and nutritive value $\left(\mathrm{LI}_{95 \%}\right)$ would maximize herbage DMI owing to the greater proportion of leaves in the grazing strata (Da Silva and Carvalho, 2005; Gregorini et al., 2011). Optimum shortterm intake rate by dairy heifers grazing guinea grass was obtained when sward intercepted $95 \%$ of the incident light (Carnevalli et al., 2006; Palhano et al., 2007). Enteric methane $\left(\mathrm{CH}_{4}\right)$ is the predominant source of GHG emissions in livestock systems (Crosson et al., 2011; Guerci et al., 2013), ranging from $30 \%$ (high feed concentrate levels) to $83.5 \%$ (pasture-based) of total GHG emissions in dairy farming systems (Aguirre-Villegas et al., 2017). Enteric $\mathrm{CH}_{4}$ production from animal digestion is associated with feed intake and herbage chemical composition (Janssen, 2010). In temperate grasslands, grazing strategies can be used to reduce the $\mathrm{CH}_{4}$ emission intensity (i.e. $\mathrm{CH}_{4} / \mathrm{kg}$ of product) and $\mathrm{CH}_{4}$ yield (i.e. $\mathrm{CH}_{4} / \mathrm{kg}$ of DMI) (Wims et al., 2010; Boland et al., 2013; Muñoz et al., 2016).

Although the studies aforementioned have demonstrated the benefits of grazing strategies based on $\mathrm{LI}_{95 \%}$ criteria, most focused solely on plant responses. There is a knowledge gap in relationships among plant and animal responses and environmental benefits in tropical pasture-based dairy systems. The central hypothesis of this study is that the change in sward structure caused by $\mathrm{LI}_{95 \%}$ management would optimize processes related to plant growth, plant-animal interface and between animal-rumen microorganisms delivering improved environmental services to the system by reducing $\mathrm{CH}_{4}$ emission intensity and increased milk productivity. Our objective was to investigate the 
influence of strategic grazing with pre-grazing targets ( $\mathrm{LI}_{95 \%}$ and $\mathrm{LI}_{\mathrm{Max}}$ ) on enteric $\mathrm{CH}_{4}$ emissions and animal productivity in dairy tropical pasture of elephant grass (Pennisetum purpureum Schum. cv. Cameroon).

\subsection{Material and Methods}

All procedures for this study were approved by the Animal (15.5.1246.11.2) and Environment Ethics Committees (17.5.999.11.9) at the University of São Paulo, College of Agriculture "Luiz de Queiroz" (USP/ESALQ).

\subsubsection{Study site}

The experiment was conducted in Piracicaba, SP, Brazil $\left(22^{\circ} 42^{\prime} \mathrm{S}, 47^{\circ} 38^{\prime} \mathrm{W}\right.$ and 546 a.s.1.) on a rainfed, non-irrigated elephant grass (Pennisetum purpureum Schum. cv. Cameroon) pasture established in 1972 in a high fertility Eutroferric Red Nitossol (Pereira et al., 2014). The climate is sub-tropical with dry winters and $1328 \mathrm{~mm}$ average annual rainfall (CEPAGRI, 2012). The lowest and highest mean temperatures were recorded in July $\left(19.7^{\circ} \mathrm{C}\right)$ and December $\left(27.1^{\circ} \mathrm{C}\right)$, respectively. The greatest accumulated rainfall was observed from late spring to summer (1090 mm from November 2015 to March 2016), and the lowest from winter to early spring (356 $\mathrm{mm}$ from June to October 2015).

\subsubsection{Treatments and experimental design}

The two treatments were pre-grazing targets of either $95 \%$ or maximum canopy light interception during regrowth ( $\mathrm{LI}_{95 \%}$ and $\mathrm{LI}_{\mathrm{Max}}$, respectively). Treatments were allocated to experimental units (2058 $\mathrm{m}^{2}$ paddocks) according to a randomized complete block design, with six replications. The slope and chemical soil characteristics were considered as blocking criteria.

Before treatment implementation, paddocks were grazed and mowed to $45 \mathrm{~cm}$ for standardization in mid-January 2015. The pre-grazing targets of $\mathrm{LI}_{95 \%}$ and $\mathrm{LI}_{\mathrm{Max}}$ were maintained until late November 2015 (adaptation period). This period was necessary to adapt sward structure to treatments and to identify the pre-grazing sward surface height ( $\mathrm{SSH})$ for the pre-grazing targets ( $\mathrm{LI}_{95 \%}$ and $\mathrm{LI}_{\mathrm{Max}}$ ). For both treatments, the herbage depletion level (HDL) corresponded to $50 \%$ of the pregrazing SSH as a means to maintain high short-term rates of herbage intake (Fonseca et al., 2012; Carvalho, 2013). The pre- and post-grazing SSH were measured from ground level to the top leafy horizon by 40 systematic readings per paddock, using a stick graduated in centimeters. Canopy LI was 
monitored using a LAI 2000 canopy analyzer (LI-COR, Lincoln, NE, USA) to take six readings above the canopy and thirty at ground level per experimental unit (Pereira et al., 2014).

Measurements were performed after the adaptation period throughout the experimental period (from December 4th 2015 to April 3th 2016 - 119 days), which was divided into three sampling periods of forty days (early summer, full summer and late summer). During the experimental period, pre-grazing targets for grazing management treatments were based on the heights corresponding to the LI treatments determined during the adaptation period. A total of $215 \mathrm{~kg} \mathrm{~N} / \mathrm{ha}$ (as urea, $45 \%$ of $\mathrm{N}$ ) was applied throughout the experimental period. Because the grazing interval was not constant (as a consequence of experimental treatments design), the total amount of $\mathrm{N}$ to be applied was divided throughout the experimental period (119 days) and a daily rate of nitrogen fertilization was calculated. The amount of $\mathrm{N}$ applied per paddock after each grazing was proportional to the length of the corresponding rest period (daily rate $\times$ rest period), ensuring similar $\mathrm{N}$ fertilization to both treatments at the end of the experimental period (Da Silva et al., 2017).

\subsubsection{Plant measurements}

Frequency of tussocks, bare ground, and weeds as well as tussock perimeter were measured five times throughout the adaptation and experimental periods. At the beginning of a regrowth cycle, a nylon string transect was placed within the paddock, with readings taken to identify botanical composition every two meters. Tussocks present at each point had their perimeter measured at ground level using a metric tape. A total of 100-points were sampled per paddock and the frequency of each botanical component was calculated as a proportion of the total number of reading points (Pereira et al., 2015a). At the last evaluation of botanical composition, tiller population density was determined by counting the total number of tillers in three rectangular sub-samples $\left(0.94 \mathrm{~m}^{2}\right.$ each $)$ randomized per paddock.

At the beginning of the experimental period, each paddock was divided up into three subpaddocks $\left(686 \mathrm{~m}^{2}\right)$ with plant measurements performed within the central sub-paddock. The SSH was measured as described above with 40 readings per sub-paddock. Pre-grazing herbage mass was quantified in each grazing cycle from three rectangular samples collected randomly $\left(0.94 \mathrm{~m}^{2}\right.$ each $)$ from each sub-paddock. The herbage was clipped above post-grazing SSH according to each treatment, weighed fresh, and two sub-samples taken to the laboratory. One sub-sample was used to determine plant-part components by hand separation into leaf (leaf blades), stem (stems + leaf sheaths) and dead material. The second sub-sample was used to determine herbage chemical composition. Both samples were dried in a forced-air drier at $65{ }^{\circ} \mathrm{C}$ to constant weight. Herbage and morphological components accumulation represent the sum of pre-grazing herbage mass throughout the experimental period. Samples to determine herbage chemical composition were ground through a $1-\mathrm{mm}$ screen 
(Wiley Mill, Thomas Scientific, Philadelphia, PA). Dry matter (DM) and ash concentrations were determined at $105{ }^{\circ} \mathrm{C}$ for $24 \mathrm{~h}$ and $600{ }^{\circ} \mathrm{C}$ for $4 \mathrm{~h}$, respectively (AOAC International, 2005). Neutral detergent fiber (NDF), acid detergent fiber (ADF) and lignin concentrations were determined sequentially (Van Soest et al., 1991). Total nitrogen (N) concentration was determined by the Dumas combustion method using N analyzer (Leco FP-2000 N Analyzer; Leco Instruments Inc., St. Joseph, MI, USA), and crude protein (CP) concentration calculated as $\mathrm{N} \times 6.25$.

Grazing losses were estimated from two randomized samples $\left(0.94 \mathrm{~m}^{2}\right.$ each) per paddock. At pre-grazing, rectangular frames were placed on the soil surface and all litter removed leaving a clean soil surface. After grazing, these areas were revisited and all material lying on the ground as well as broken stems and green leaves still attached and hanging on tussocks were collected, weighed fresh, and dried in a forced-air drier at $65^{\circ} \mathrm{C}$ to constant weight (Silveira et al., 2013). Grazing losses were expressed in DM/ha and as a percentage of the pre-grazing herbage mass (above post-grazing SSH) and its complement to 100 was considered as grazing efficiency (Carnevalli et al., 2006). Herbage and leaf allowance were calculated by the relationship between pre-grazing herbage mass (above postgrazing SSH) and number of cows per day (Pérez-Prieto and Delagarde, 2013).

\subsubsection{Herd and feeding}

Twenty-six Holstein $\times$ Jersey dairy cows averaging $488 \pm 60 \mathrm{~kg}$ body weight $(\mathrm{BW})$ (mean \pm SD), $2.94 \pm 0.18$ body condition score (BCS), daily milk yield of $20.3 \pm 2.6 \mathrm{~kg} / \mathrm{d}$, and $126 \pm 90$ days in milk (DIM) were stratified and grouped in pairs into 13 blocks according to daily milk yield and DIM, and then randomly assigned to either $\mathrm{LI}_{95 \%}$ and $\mathrm{LI}_{\mathrm{Max}}$ grazing management. An additional herd of drycows (10 to 13 cows) was maintained in an adjacent area of elephant grass and was used to keep grazing management targets constant, as needed. The stocking rate was calculated by number of cows used daily for each treatment, considering experimental cows and the additional herd.

Concentrate meals were fed individually twice daily (4:30 am and 2:30 pm) before milking ( $5 \mathrm{am}$ and $3 \mathrm{pm}$ ) at a rate of $1 \mathrm{~kg}$ of concentrate $3 \mathrm{~kg}$ of milk (considering the average of each block). The rate was established based on milk yield at the beginning of each sampling period (Danes et al., 2013). The concentrate meal was composed of citrus pulp (35\%), corn gluten feed (30\%), fine ground corn $(20 \%)$, soybean meal (10\%) and mineral (5\%), with chemical composition as followed: $88.4 \%$ DM, $10.3 \%$ ash, $14.0 \% \mathrm{CP}, 22.2 \% \mathrm{NDF}, 9.3 \% \mathrm{ADF}, 3.3 \%$ ether extract and $49.8 \%$ non-fibrous carbohydrate. 


\subsubsection{Animal measurements}

The BW and BCS were measured at the end of each sampling period over three consecutive days (Edmonson et al., 1989). Milk yield was recorded daily with samples collected in vials containing bronopol preservative pill and analyzed for fat, protein, lactose, and milk solids using infrared procedures (MilkoScan FT+; Foss North America Inc., Eden Prairie, MN).

Herbage intake was estimated from total fecal excretion and feed indigestibility. To estimate total fecal excretion, titanium dioxide $\left(\mathrm{TiO}_{2}\right)$ was dosed twice a day $(20 \mathrm{~g} / \mathrm{cow}$ per day) after concentrate meals over 12 days. Fecal samples were collected from the rectum after concentrate meals on the last 5 days, dried in a forced-air drier at $55{ }^{\circ} \mathrm{C}$ for $72 \mathrm{~h}$, ground through a $1-\mathrm{mm}$ screen (WileyMill, Thomas Scientific, Philadelphia, PA) and composited forming one sample per sampling period by cow. Titanium dioxide concentration in feces was determined according to Myers et al. (2004). To determine the feed indigestibility, the indigestible NDF (iNDF) content of herbage, concentrate, and fecal samples were estimated by $240 \mathrm{~h}$ in vitro incubation (Goeser and Combs, 2009). Total fecal excretion, fecal excretion from concentrate, and herbage intake were calculated according to De Souza et al. (2015).

Enteric $\mathrm{CH}_{4}$ emissions were estimated using sulfur hexafluoride $\left(\mathrm{SF}_{6}\right)$ as tracer gas (Johnson and Johnson, 1995). Pre-calibrated permeation tubes containing $\mathrm{SF}_{6}$ with known release rates (1.41 \pm $0.40 \mathrm{mg} /$ day) were placed into the rumen of each cow $72 \mathrm{~h}$ prior to the first collection. Sampling apparatus included a PVC collection canister $(2.3 \mathrm{~L})$ and adjustable halter containing stainless steel capillary tubing and brass connections. The cows were adapted to the sampling apparatus over 7 days prior to collection with $\mathrm{CH}_{4}$ emissions measured at 24-hour intervals over 7 consecutive days. Canisters were vacuumed to approximately -13.5 psi using a three-stage vacuum pump (Symbol, Sumaré, SP, Brazil) and Druck DPI 705 digital manometer (GE Druck, South Burlington, VT, EUA) and replaced daily just after the afternoon concentrate meal. Background $\mathrm{SF}_{6}$ and $\mathrm{CH}_{4}$ concentrations were determined using two sampling apparatus placed daily in the field near the grazing herd. Methane and $\mathrm{SF}_{6}$ concentrations were determined at the Laboratory of Biogeochemistry and Tracer Gases Analysis (Embrapa Meio Ambiente, Jaguariúna, SP, BRA) using gas chromatography (HP6890, Agilent, Delaware, USA). Prior to chromatograph determination, canisters were pressurized to $1.3-1.5$ psi with ultrapure nitrogen 5.0, and pressures recorded by Druck DPI 705 digital manometer (GE Druck, South Burlington, VT, EUA) in order to calculate the dilution factor. The chromatograph was equipped with a flame ionization detector (FID) at $280{ }^{\circ} \mathrm{C}$ for $\mathrm{CH}_{4}$ (column megabore, $0.53 \mathrm{~mm} \times 30$ $\mathrm{m} \times 15 \mu \mathrm{m}$, Plot $\mathrm{HP}-\mathrm{Al} / \mathrm{M}$ ) and an electron capture detector (ECD) at $300{ }^{\circ} \mathrm{C}$ for $\mathrm{SF}_{6}$ (column megabore, $0.53 \mathrm{~mm} \times 30 \mathrm{~m} \times 25 \mu \mathrm{m}$, HP-MolSiv), with two loops of $0.5 \mathrm{~cm}^{3}$ maintained at $80{ }^{\circ} \mathrm{C}$ attached to 2 six-way valves. Calibration curves were established using standard certified gases for $\mathrm{CH}_{4}(4.85 \pm 5 \% ; 9.96 \pm 1.65 \%$ and $19.1 \pm 3.44 \% \mathrm{ppm})$ and $\mathrm{SF}_{6}(34.0 \pm 9.0 ; 91.0 \pm 9.0$ and $978.0 \pm$ 98.0 ppt) (Westberg et al., 1998). Daily methane emission was calculated from collected $\mathrm{SF}_{6}$ and $\mathrm{CH}_{4}$ 
concentrations in the canisters discounting background concentrations, and the value of $\mathrm{SF}_{6}$ permeation tube release rate (Johnson and Johnson, 1995).

\subsubsection{Statistical analysis}

Analysis of variance was performed using the Mixed Procedure (SAS 9.3; SAS Institute Inc., Cary, NC). Different structures of the variance-covariance matrices were tested and the Bayesian Information Criterion was adopted to select the best fit matrix. Within plant parameters, the paddock was considered the experimental unit, and for animal measurements, the cow was considered the experimental unit. Blocks were considered random terms, and LI, sampling periods and their interactions were treated as fixed effects. Sampling periods were treated as repeated measures. For tussock measurements, season of the year was considered a fixed effect because assessments were made throughout the entire study period (adaptation + experimental). Means were calculated using the LSMEANS statement, compared using the Student's t-test and the Bonferroni adjustment. Differences were declared significant at $P \leq 0.05$, and trends were declared at $P \leq 0.10$.

\subsection{Results}

\subsubsection{Canopy light interception and sward surface height}

Grazing management targets and sward characteristics during the adaptation and experimental periods are presented in Table 1 . The $\mathrm{LI}_{95 \%}$ pre-grazing target was reached at $99 \mathrm{~cm}$ $(\approx 100 \mathrm{~cm})$ and the $\mathrm{LI}_{\mathrm{Max}}$ pre-grazing target was reached at $134.5 \mathrm{~cm}(\approx 135 \mathrm{~cm})$. For both treatments, HDL was close to the target of $50 \%$ of the pre-grazing SSH, and corresponded to post-grazing heights of 50.4 and $64.3 \mathrm{~cm}$ for $\mathrm{LI}_{95 \%}$ and $\mathrm{LI}_{\mathrm{Max}}$, respectively. 
Table 1. Canopy light interception, pre- and post- grazing sward surface height (SSH) and herbage depletion level (HDL) of elephant grass subjected to strategies of rotational stocking management ( $\mathrm{LI}_{95 \%}$ or $\mathrm{LI}_{\mathrm{Max}}$ ) during the adaptation (Jan-Nov 2015) and experimental (Dec 2015-Apr 2016) periods $(\mathrm{n}=6)$

\begin{tabular}{|c|c|c|c|c|}
\hline \multirow{2}{*}{$\begin{array}{l}\text { Period } \\
\text { Adaptation }\end{array}$} & \multicolumn{2}{|c|}{ Treatments } & \multirow[t]{2}{*}{ SEM $^{1}$} & \multirow[t]{2}{*}{$P$-value } \\
\hline & $\mathbf{L I}_{95 \%}$ & $\mathbf{L I}_{\text {Max }}$ & & \\
\hline Light interception, \% & 95.2 & 98.0 & 0.11 & $<0.0001$ \\
\hline Pre-SSH, cm & 99.0 & 134.5 & 0.94 & $<0.0001$ \\
\hline Post-SSH, cm & 50.4 & 64.3 & 0.86 & $<0.0001$ \\
\hline HDL, $\%$ of Pre-SSH & 49.0 & 50.8 & 0.57 & 0.03 \\
\hline \multicolumn{5}{|l|}{ Experimental } \\
\hline Pre-SSH, cm & 99.7 & 134.4 & 0.58 & $<0.0001$ \\
\hline Post-SSH, cm & 50.9 & 68.3 & 0.45 & $<0.0001$ \\
\hline HDL, $\%$ of Pre-SSH & 49.1 & 48.9 & 0.46 & 0.7269 \\
\hline
\end{tabular}

\subsubsection{Canopy cover}

Frequencies of tussocks and bare ground, and tussock perimeter were affected by season $(P$ $<0.01$ ) indicating a strong effect of growth conditions on plant ecophysiology (Table 2). Across seasons of the year, tussocks showed a tendency of greater frequency $(P=0.06)$ for $\mathrm{LI}_{95 \%}$ than $\mathrm{LI}_{\mathrm{Max}}$ (38\% and 33\%, respectively). Inversely, the frequency of bare ground was greater $(P=0.04)$ for $\mathrm{LI}_{\mathrm{Max}}$ than $\mathrm{LI}_{95 \%}$ (54\% and 50\%, respectively). There was no effect of LI pre-grazing on tussock perimeter. However, in the second summer, tussocks under $\mathrm{LI}_{95 \%}$ management had a greater perimeter than $\mathrm{LI}_{\mathrm{Max}}$ $(P<0.05)$. Tiller population density was greater $(P<0.01)$ for $\mathrm{LI}_{95 \%}$ relative to $\mathrm{LI}_{\text {Max }}$.

Table 2. Frequencies of tussock and bare ground, tussock perimeter and tiller population density of elephant grass subjected to strategies of rotational stocking management $\left(\mathrm{LI}_{95 \%}\right.$ or $\left.\mathrm{LI}_{\mathrm{Max}}\right)$ during the adaptation (Jan-Nov 2015) and experimental (Dec 2015-Apr 2016) periods $(\mathrm{n}=6)$

\begin{tabular}{|c|c|c|c|c|c|c|c|c|c|}
\hline & \multicolumn{5}{|c|}{ Seasons $^{1}$} & \multirow[t]{2}{*}{ SEM $^{2}$} & \multicolumn{3}{|c|}{$P$-value } \\
\hline & S1 & $\mathbf{A} / \mathbf{W}$ & ES & LS & S2 & & Trt $^{3}$ & Per $^{4}$ & Trt $\times$ Per \\
\hline & \multicolumn{5}{|c|}{ Frequency of tussocks, \% } & \multirow{3}{*}{3.30} & \multirow{3}{*}{0.0633} & \multirow{3}{*}{$<0.0001$} & \multirow{3}{*}{0.6955} \\
\hline $\mathrm{LI}_{95 \%}$ & $32.8 \mathrm{Ab}$ & $33.7 \mathrm{Ab}$ & $28.6 \mathrm{Ab}$ & $35.7 \mathrm{Ab}$ & $52.9 \mathrm{Aa}$ & & & & \\
\hline $\mathrm{LI}_{\mathrm{Max}}$ & $32.9 \mathrm{Ab}$ & $29.9 \mathrm{Ab}$ & $27.5 \mathrm{Ab}$ & $26.8 \mathrm{Ab}$ & 47.7 Aa & & & & \\
\hline & \multicolumn{5}{|c|}{ Frequency of bare ground, $\%$} & \multirow{3}{*}{3.23} & \multirow{3}{*}{0.041} & \multirow{3}{*}{$<0.0001$} & \multirow{3}{*}{0.8760} \\
\hline $\mathrm{LI}_{95 \%}$ & $54.0 \mathrm{Aa}$ & $54.9 \mathrm{Aa}$ & $59.7 \mathrm{Aa}$ & $51.8 \mathrm{Aa}$ & $28.8 \mathrm{Ab}$ & & & & \\
\hline $\mathrm{LI}_{\mathrm{Max}}$ & 56.2 Aa & $57.4 \mathrm{Aa}$ & $62.2 \mathrm{Aa}$ & $60.5 \mathrm{Aa}$ & $32.4 \mathrm{Ab}$ & & & & \\
\hline & \multicolumn{5}{|c|}{ Tussock perimeter, cm } & \multirow{3}{*}{9.15} & \multirow{3}{*}{0.795} & \multirow{3}{*}{$<0.0001$} & \multirow{3}{*}{0.0604} \\
\hline $\mathrm{LI}_{95 \%}$ & $174 \mathrm{Ab}$ & $186 \mathrm{Ab}$ & $193 \mathrm{Ab}$ & $165 \mathrm{Ab}$ & $236 \mathrm{Aa}$ & & & & \\
\hline $\mathrm{LI}_{\text {Max }}$ & $185 \mathrm{Aa}$ & $186 \mathrm{Aa}$ & $183 \mathrm{Aa}$ & $187 \mathrm{Aa}$ & $205 \mathrm{Ba}$ & & & & \\
\hline & \multicolumn{5}{|c|}{ Tiller population density, tiller/m² } & \multirow{3}{*}{6.53} & \multirow{3}{*}{0.0049} & \multirow{3}{*}{ - } & \multirow{3}{*}{ - } \\
\hline $\mathrm{LI}_{95 \%}$ & - & - & - & - & 129.1 & & & & \\
\hline $\mathrm{LI}_{\mathrm{Max}}$ & - & - & - & - & 87.3 & & & & \\
\hline \multicolumn{10}{|c|}{$\begin{array}{l}\text { Means followed by the same capital letter in columns and the lower case letter in rows do not differ }(P>0.05) \\
\text { 'S1: Summer } 1 \text { - Mar 2015, A/W: Autumn/Winter - Jun/Jul 2015, ES: Early Spring - Sep 2015, LS: Late Spring - Nov } 2015 \text { and } \\
\text { S2: Summer } 2 \text { - Dec 2015 } \\
\text { 2Standard error of the mean } \\
\text { 'Treatment effect } \\
{ }^{4} \text { Sampling period effect }\end{array}$} \\
\hline
\end{tabular}




\subsubsection{Herbage characteristics}

The $\mathrm{LI}_{95 \%}$ provided more grazing cycles $(P<0.01)$ associated with lower stocking and shorter rest periods than $\mathrm{LI}_{\text {Max }}(P<0.01)$ (Table 3). Longer rest periods of $\mathrm{LI}_{\mathrm{Max}}$ allowed greater plant growth and determined higher pre-grazing herbage mass $(P<0.01)$ with more stems $(P<0.01)$, lower leaf blade $(P<0.01)$ and lower leaf:stem ratio $(P<0.01)$ than $\mathrm{LI}_{95 \%}$. There was no treatment effect on dead material $(P=0.31)$. Herbage accumulation was not affected by LI strategy $(P=0.11)$ but more frequent defoliation $\left(\mathrm{LI}_{95 \%}\right)$ resulted in greater leaf $(P=0.01)$ and lower stem $(P<0.01)$ accumulation throughout the experimental period (Table 3). Daily herbage allowances were greater for $\mathrm{LI}_{\mathrm{Max}}$ than $\mathrm{LI}_{95 \%}(P=0.02)$. The $\mathrm{LI}_{95 \%}$ promoted lower grazing losses $(P<0.01)$ and more efficient grazing $(P<$ 0.01) than $\mathrm{LI}_{\mathrm{Max}}$. Grazing management strategy influenced herbage chemical composition, with $\mathrm{LI}_{95 \%}$ herbage having greater $\mathrm{CP}(P<0.01)$, and lower $\operatorname{ADF}(P=0.03)$ and lower lignin $(P<0.01$; Table 3$)$. There was no treatment effect on DM $(P=0.70)$, NDF $(P=0.11)$, and ash $(P=0.28)$ (Table 3$)$.

Table 3. Grazing cycles, stocking period, rest period, pre-grazing herbage characteristics, herbage accumulation, herbage allowance, grazing losses, grazing efficiency and herbage chemical composition of elephant grass subjected to strategies of rotational stocking management $\left(\mathrm{LI}_{95 \%}\right.$ or $\left.\mathrm{LI}_{\mathrm{Max}}\right)$ during the experimental period (Dec 2015-Apr 2016) ( $\mathrm{n}=6)$

\begin{tabular}{|c|c|c|c|c|c|c|}
\hline \multirow[t]{2}{*}{ Item } & \multicolumn{2}{|c|}{ Treatments } & \multirow[t]{2}{*}{ SEM $^{1}$} & \multicolumn{3}{|c|}{$P$-value } \\
\hline & $\mathbf{L I}_{95 \%}$ & $\mathbf{L I}_{\mathbf{M a x}}$ & & Trt $^{2}$ & Per $^{3}$ & Trt $\times$ Per \\
\hline Grazing cycles, $\mathrm{n}$ & 5.6 & 3.5 & 0.16 & $<0.0001$ & 0.1330 & 0.2430 \\
\hline Stocking period, days & 1.0 & 1.4 & 0.06 & $<0.0001$ & 0.0582 & 0.0917 \\
\hline Rest period, days & 21.1 & 31.7 & 0.60 & $<0.0001$ & $<0.0001$ & 0.5215 \\
\hline Pre-grazing herbage mass ${ }^{4}, \mathrm{~kg}$ of DM/ha & 2890 & 4890 & 207.9 & $<0.0001$ & 0.3625 & 0.3410 \\
\hline Leaf blade ${ }^{4}, \%$ & 95.1 & 81.1 & 0.93 & $<0.0001$ & 0.7648 & 0.1049 \\
\hline $\mathrm{Stem}^{4}, \%$ & 3.5 & 16.6 & 0.52 & $<0.0001$ & 0.0919 & 0.2000 \\
\hline Dead material $^{4}, \%$ & 1.4 & 2.3 & 0.20 & 0.3132 & 0.0137 & 0.4464 \\
\hline Leaf : Stem ratio ${ }^{4}$ & 32.8 & 4.6 & 3.50 & $<0.0001$ & 0.0011 & 0.0004 \\
\hline Herbage accumulation $^{4}, \mathrm{~kg}$ of DM/ha & 15441 & 16683 & 847.0 & 0.1079 & - & - \\
\hline Leaf accumulation ${ }^{4}, \mathrm{~kg}$ of DM/ha & 14611 & 13276 & 730.2 & 0.0134 & - & - \\
\hline Stem accumulation ${ }^{4}, \mathrm{~kg}$ of DM/ha & 795 & 3322 & 264.2 & $<0.0001$ & - & - \\
\hline Herbage allowance ${ }^{4}, \mathrm{~kg}$ of DM/cow.day & 21.2 & 29.7 & 2.53 & 0.016 & 0.5026 & 0.4409 \\
\hline Grazing losses, $\mathrm{kg}$ of DM/ha & 292 & 1203 & 100.3 & $<0.0001$ & $<0.0001$ & $<0.0001$ \\
\hline Grazing efficiency, $\%$ & 89.3 & 79.9 & 2.88 & 0.0003 & 0.004 & 0.7755 \\
\hline \multicolumn{7}{|l|}{ Herbage chemical composition, \% DM } \\
\hline Dry matter & 19.5 & 19.2 & 0.91 & 0.6992 & 0.0724 & 0.2753 \\
\hline Crude protein & 21.0 & 19.4 & 0.50 & 0.0004 & $<0.0001$ & 0.0387 \\
\hline Neutral detergent fiber & 61.2 & 63.0 & 1.26 & 0.1121 & 0.1438 & 0.2865 \\
\hline Acid detergent fiber & 33.9 & 36.3 & 1.14 & 0.0248 & 0.2603 & 0.7348 \\
\hline Lignin & 3.3 & 3.8 & 0.16 & 0.0040 & 0.2429 & 0.4676 \\
\hline Ash & 10.4 & 11.2 & 0.54 & 0.2816 & 0.0228 & 0.6754 \\
\hline
\end{tabular}

\footnotetext{
${ }^{1}$ Standard error of the mean

${ }^{2}$ Treatment effect

${ }^{3}$ Sampling period effect

${ }^{4}$ Estimated above post-grazing SSH
} 


\subsubsection{Dry matter intake, animal performance and $\mathrm{CH}_{4}$ emissions}

Animal responses are presented in Table 4. Stocking rate was 33\% greater for $\mathrm{LI}_{95 \%}$ than $\mathrm{LI}_{\text {Max }}(P<0.01)$. Greater herbage $(P<0.01)$ and total DMI $(P<0.01)$ were observed for $\mathrm{LI}_{95 \%}$ than

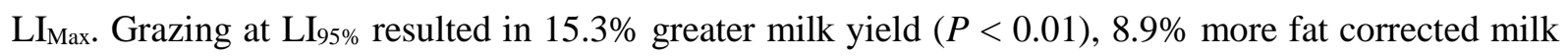
$(\mathrm{P}=0.02), 8 \%$ greater protein $(P<0.01), 15.3 \%$ more lactose $(P<0.01)$ and $6.2 \%$ greater milk solids $(P<0.01)$ yields. Fat yield was not affected by treatment $(P=0.20)$. There were no LI effects on BW and BCS changes $\left(P=0.61\right.$ and $P=0.13$, respectively). Daily enteric $\mathrm{CH}_{4}$ emission $(\mathrm{g} / \mathrm{d})$ was not affected by treatment $(P=0.85)$, however $\mathrm{LI}_{95 \%}$ grazing management increased the efficiency of milk $(P<0.01)$, fat $(P<0.01)$, protein $(P<0.01)$ and milk solids $(P<0.01)$ yields per $\mathrm{g}$ of $\mathrm{CH}_{4}$ emitted by $21 \%, 15 \%, 13 \%$ and $16 \%$, respectively. Additionally, cows grazing elephant grass managed with the $\mathrm{LI}_{95 \%}$ pre-grazing target had lower $\mathrm{CH}_{4}$ yield $(P=0.02)$.

Table 4. Stocking rate, daily dry matter intake (DMI), milk yield and enteric $\mathrm{CH}_{4}$ emissions of cows grazing elephant grass subjected to strategies of rotational stocking management ( $\mathrm{LI}_{95 \%}$ or $\mathrm{LI}_{\mathrm{Max}}$ ) during the experimental period (Dec 2015-Apr 2016) $(\mathrm{n}=$ 13)

\begin{tabular}{|c|c|c|c|c|c|c|}
\hline \multirow[t]{2}{*}{ Item } & \multicolumn{2}{|c|}{ Treatments } & \multirow[t]{2}{*}{ SEM $^{1}$} & \multicolumn{3}{|c|}{$P$-value } \\
\hline & $\mathbf{L I}_{\mathbf{9 5} \%}$ & $\mathbf{L I}_{\text {Max }}$ & & Trt $^{2}$ & Per $^{3}$ & Trt $\times$ Per \\
\hline Stocking rate, cows/ha & 9.3 & 7.0 & 0.98 & 0.0054 & - & - \\
\hline \multicolumn{7}{|l|}{ Daily DMI, kg of DM/cow } \\
\hline Herbage & 12.3 & 10.1 & 0.52 & 0.0017 & 0.0071 & 0.1781 \\
\hline Total & 18.2 & 15.9 & 0.61 & 0.0028 & 0.1113 & 0.2177 \\
\hline \multicolumn{7}{|l|}{ Yield, kg/d } \\
\hline Milk & 18.1 & 15.7 & 1.01 & $<0.0001$ & $<0.0001$ & 0.7768 \\
\hline $3.5 \% \mathrm{FCM}^{4}$ & 18.4 & 16.9 & 1.18 & 0.0205 & 0.0156 & 0.6329 \\
\hline Fat & 0.646 & 0.608 & 0.0407 & 0.2004 & 0.0709 & 0.355 \\
\hline Protein & 0.556 & 0.515 & 0.0274 & 0.0098 & 0.001 & 0.8361 \\
\hline Lactose & 0.792 & 0.687 & 0.0461 & $<0.0001$ & $<0.0001$ & 0.5586 \\
\hline Milk solids & 2.07 & 1.95 & 0.1084 & 0.0059 & 0.0004 & 0.7953 \\
\hline $\mathrm{BW}^{5}$ change, $\mathrm{kg} / \mathrm{d}$ & 0.4375 & 0.5530 & 0.36 & 0.6107 & 0.0031 & 0.6086 \\
\hline $\mathrm{BCS}^{6}$ change & -0.06 & 0.01 & 0.05 & 0.1330 & 0.8276 & 0.7137 \\
\hline \multicolumn{7}{|l|}{$\mathrm{CH}_{4}$ emissions } \\
\hline $\mathrm{g} / \mathrm{d}$ & 297.8 & 296.1 & 13.30 & 0.8533 & $<0.0001$ & 0.8978 \\
\hline $\mathrm{g} / \mathrm{kg}$ of milk yield & 16.2 & 20.5 & 1.09 & $<0.0001$ & $<0.0001$ & 0.1365 \\
\hline $\mathrm{g} / \mathrm{kg}$ of fat yield & 438.9 & 515.3 & 24.71 & 0.0005 & $<0.0001$ & 0.9986 \\
\hline $\mathrm{g} / \mathrm{kg}$ of protein yield & 525.2 & 604.6 & 22.40 & $<0.0001$ & $<0.0001$ & 0.1931 \\
\hline $\mathrm{g} / \mathrm{kg}$ of milk solids yield & 133.5 & 159.5 & 6.71 & $<0.0001$ & $<0.0001$ & 0.4666 \\
\hline $\mathrm{g} / \mathrm{kg}$ of dry matter intake & 20.2 & 24.7 & 1.33 & 0.0199 & $<0.0001$ & 0.0012 \\
\hline 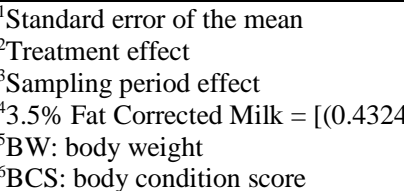 & & & & & & \\
\hline
\end{tabular}




\subsubsection{Milk yield and $\mathrm{CH}_{4}$ emissions per hectare}

Milk yield and $\mathrm{CH}_{4}$ emissions per hectare are presented in Table 5. Grazing at $\mathrm{LI}_{95 \%}$ increased milk yield by $51 \%(P<0.01)$, fat yield by $42 \%(P=0.02)$, protein yield by $41 \%(P<0.01)$ and milk solids yield by $40 \%(P<0.01)$ per hectare. The enteric $\mathrm{CH}_{4}$ emitted per hectare was $29 \%$ greater for $\mathrm{LI}_{95 \%}$ than $\mathrm{LI}_{\text {Max }}(P<0.01)$. Additionally, $\mathrm{LI}_{95 \%}$ pre-grazing target increased productivity of milk $(P<0.01)$, protein $(P<0.01)$ and milk solids $(P<0.01)$ per $\mathrm{kg}$ of $\mathrm{CH}_{4}$ released per hectare by $16 \%, 9 \%$ and $7 \%$, respectively (Table 5).

Table 5. Milk yield and $\mathrm{CH}_{4}$ emissions per hectare (ha) of cows grazing elephant grass subjected to strategies of rotational stocking management $\left(\mathrm{LI}_{95 \%}\right.$ or $\mathrm{LI}_{\mathrm{Max}}$ ) during the experimental period (Dec 2015-Apr 2016) $(\mathrm{n}=6)$

\begin{tabular}{|c|c|c|c|c|}
\hline \multirow[t]{2}{*}{ Item } & \multicolumn{2}{|c|}{ Treatments } & \multirow[t]{2}{*}{ SEM $^{1}$} & \multirow[t]{2}{*}{$P$-value } \\
\hline & $\mathbf{L I}_{\mathbf{9 5} \%}$ & $\mathbf{L I}_{\text {Max }}$ & & \\
\hline Milk, kg/ha.day & 169.8 & 112.4 & 20.40 & 0.0012 \\
\hline Fat, kg/ha.day & 6.1 & 4.3 & 0.71 & 0.0148 \\
\hline Protein, kg/ha.day & 5.2 & 3.7 & 0.62 & 0.0026 \\
\hline Milk solids, $\mathrm{kg} / \mathrm{h}$.day & 19.5 & 13.9 & 2.42 & 0.0057 \\
\hline $\mathrm{CH}_{4}, \mathrm{~kg} / \mathrm{ha}$ per day & 2.7 & 2.1 & 0.24 & 0.0055 \\
\hline \multicolumn{5}{|l|}{ Productivity vs. $\mathrm{CH}_{4}$} \\
\hline Milk, $\mathrm{kg} / \mathrm{kg}$ of $\mathrm{CH}_{4} / \mathrm{ha}$.day & 62.2 & 53.8 & 4.79 & 0.0002 \\
\hline Fat, $\mathrm{kg} / \mathrm{kg}$ of $\mathrm{CH}_{4} / \mathrm{ha}$.day & 2.22 & 2.06 & 0.132 & 0.1151 \\
\hline Protein, $\mathrm{kg} / \mathrm{kg}$ of $\mathrm{CH}_{4} / \mathrm{ha}$.day & 1.91 & 1.76 & 0.135 & $<0.0001$ \\
\hline Milk solids, $\mathrm{kg} / \mathrm{kg}$ of $\mathrm{CH}_{4} / \mathrm{ha}$.day & 7.13 & 6.66 & 0.550 & 0.0098 \\
\hline
\end{tabular}

\subsection{Discussion}

Grazing strategies that maintain shorter rather than taller pre-grazing SSH normally result in greater canopy cover, associated with greater frequency of tussocks and tiller population density (Pereira et al., 2015a). Greater canopy cover by plants reduces nutrient and soil transport avoiding contamination and sedimentation of waterways (McDowell and Houlbrooke, 2009). Furthermore, competition for light is stronger in taller swards, resulting in tiller death, reduced tillering, less stability of plant population, greater frequency of bare ground and reduced sward perennation (Pereira et al., 2015a). The differences observed in tussock perimeter during the second summer, one year after the adaptation period, indicate that elephant grass adapts its horizontal structure to defoliation regimes slowly. This feature is an important aspect to be considered for planning of field experiments and management strategies (Pereira et al., 2015a).

The $\mathrm{LI}_{95 \%}$ pre-grazing target results in maximum net herbage accumulation rate and is considered the critical LAI (Brougham, 1958). For tropical forage, this condition is associated with the beginning of marked stem elongation (Da Silva et al., 2015). Pre-grazing SSH is strongly correlated with LAI and LI, and can be used as a reliable field indicator for controlling herbage regrowth. 
Grazing strategies based on the maximum LI pre-grazing target result in longer regrowth periods, increasing light competition within the sward, shifting the plant's growth pattern to prioritize stem elongation and resulting in greater stem and lower leaf accumulation (Da Silva et al., 2009). In the present study, the 3-percentual unit increase in LI pre-grazing for the $\mathrm{LI}_{\mathrm{Max}}$ relative to the $\mathrm{LI}_{95 \%}$ target resulted in 50\% increase of the rest period. Total herbage accumulation was similar between treatments. However, $\mathrm{LI}_{95 \%}$ increased leaf accumulation by $10 \%$ and decreased stem accumulation by 76\% compared to $\mathrm{LI}_{\mathrm{Max}}$, as previously shown (Carnevalli et al., 2006; Barbosa et al., 2007; Silveira et al., 2013; Pereira et al., 2014).

In pasture-based systems, only a portion of the herbage accumulated is consumed by grazing animals. The remaining fraction is lost as a consequence of trampling and characterizes grazing inefficiency (Carnevalli et al., 2006; Silveira et al., 2013). Grazing at $\mathrm{LI}_{\mathrm{Max}}$ resulted in fourfold higher grazing losses than $\mathrm{LI}_{95 \%}$. This suggests that taller swards result in greater grazing losses, as observed by previous studies in tropical conditions (Carnevalli et al., 2006; Silveira et al., 2013). Inversely, the grazing efficiency was ten percentage units higher for $\mathrm{LI}_{95 \%}$ than $\mathrm{LI}_{\mathrm{Max}}$, corroborating the findings of Carnevalli et al. (2006) and Silveira et al. (2013).

The stocking rate was 33\% greater for $\mathrm{LI}_{95 \%}$ than $\mathrm{LI}_{\text {Max }}$. Voltolini et al. (2010) and Gimenes et al. (2011) found stocking rate increases ranging from 10 to $42 \%$ in elephant grass and palisade grass pastures adopting the $95 \%$ pre-grazing target of canopy light interception under rotational grazing management. Greater stocking rates are supported by greater leaf accumulation associated with lower grazing losses determining greater grazing efficiency. In the present study, $\mathrm{LI}_{95 \%}$ had $10 \%$ greater leaf accumulation associated with fourfold reduction in grazing losses. The lower grazing efficiency observed in taller swards may also be associated with higher stem accumulation and increased senescence and dead material (Pereira et al., 2014; Pereira et al., 2015b), which are plant-part components avoided by grazers (Trindade et al., 2007).

Chemical composition of the herbage is a function of the proportions of plant-part components and their tissue anatomy (Moore, 1994). In the present study, stem proportions were 3.5\% for $\mathrm{LI}_{95 \%}$ and $16.6 \%$ for $\mathrm{LI}_{\mathrm{Max}}$ and, leaf blade was $95.1 \%$ for $\mathrm{LI}_{95 \%}$ and $81.1 \%$ for $\mathrm{LI}_{\mathrm{Max}}$. The $\mathrm{ADF}$ is composed of cellulose and lignin, present mainly in the cell wall, associated with structural support for plant organs (Moore and Jung, 2001). Stems contain a higher proportion of cell wall tissues and less photosynthetic tissues than leaves (Wilson and Kennedy, 1996). On the other hand, most protein compounds are present in leaves, with the majority associated with photosynthetic enzymes (Gastal and Durand, 2000).

Daily herbage intake is determined by interactions between sward structure and grazing animals (Wade and Carvalho, 2000). Poppi et al. (1987) suggested that herbage intake by grazing animals follows an asymptotic distribution represented by two distinct phases. In the first ascending phase, herbage intake is related to sward structure (herbage or leaf mass, pre-SSH, leaf-to-stem ratio) and grazing behavior (grazing time, diet selection, bite mass and bite rate), which are characteristics 
strongly affected by grazing management strategies (Da Silva and Carvalho, 2005). In the second asymptotic phase, nutritional factors such as herbage chemical composition, time of herbage retention in the rumen and concentration of metabolic compounds are more relevant in controlling intake (Poppi et al., 1987). In the present study, it is likely that sward structure characteristics, such as pre-grazing SSH and plant-part components strongly affected grazing behavior and ultimately herbage DMI. Swards constantly kept at taller heights (such as $\mathrm{LI}_{\mathrm{Max}}$ swards) result in lower short-term intake rate at the beginning of grazing, due to the excessive length of leaf blade associated with lower bulk density of herbage in the upper strata which increases time per bite (Palhano et al., 2007; Fonseca et al., 2013; Carvalho, 2013). At the end of grazing, greater proportion of stems results in physical constraints reducing herbage intake (Laca and Lemaire, 2000; Fonseca et al., 2012; Carvalho, 2013). At the rumen level, more fibrous herbage (i.e. higher NDF, ADF and lignin) is associated with greater ruminal retention time, lower fermentation and passage rate, and lower herbage intake (Mertens, 1994; Allen, 1996; Allen, 2000; Forbes, 2007).

Herbage DMI is a key determinant of performance of livestock-based systems (Mertens, 1994; Poppi et al., 1997; Sollenberger and Burns, 2001; Coleman and Moore, 2003; Sollenberger and Vanzant, 2011). Coleman and Moore (2003) described that the combination of herbage chemical composition (i.e. nutritive value), nutrient availability (i.e. digestibility), and intake determines herbage quality (i.e. feed value) and is accepted as an indicator of animal performance. Our results indicate that it is possible to produce herbage with high nutritive value resulting in greater herbage DMI, and milk yield through pre-grazing SSH that avoids excessive stem elongation (LI ${ }_{95} \%$ ). These results corroborate those of experiments conducted in temperate climates that determined greater nutritive value, DMI and milk yield in low herbage mass swards compared to high herbage mass swards (Wims et al., 2010; Muñoz et al., 2016). In the present study, greater milk yield was observed at higher stocking rates and lower herbage allowance ( $\mathrm{LI}_{95 \%}$ ) demonstrating that in tropical swards, the distribution and arrangement of above-ground plant parts (i.e. sward structure, see Laca and Lemaire, 2000) plays a more important role than herbage allowance in determining herbage DMI and animal performance.

Enteric $\mathrm{CH}_{4}$ is affected by the amount and nature of feed, and the extent of its degradation, which in turn determines the amount of hydrogen formed in the rumen (Janssen, 2010). Although DMI and herbage nutritive value have been affected by targets of pre-grazing LI, daily enteric $\mathrm{CH}_{4}$ emission was not. Similarly, studies with temperate grasses did not observe differences in daily enteric $\mathrm{CH}_{4}$ emission from beef heifers (Boland et al., 2013) and dairy cows (Wims et al., 2010; Muñoz et al., 2016) grazing low herbage mass and high herbage mass swards, even with significant differences reported in DMI and nutritive value. The model proposed by Janssen (2010) suggests that greater passage rates increase hydrogen concentration in the rumen. Consequently, microorganisms would select pathways thermodynamically more favorable to this condition, which produce less hydrogen resulting in less $\mathrm{CH}_{4}$ formed per unit of feed ingested. It is possible that for the DMI ranges observed 
in the present study, reductions in $\mathrm{CH}_{4}$ yield $(\mathrm{g} / \mathrm{kg}$ of DMI) have compensated the greater DMI, decreasing daily $\mathrm{CH}_{4}$ emissions. Our results indicate reductions around $20 \%$ for emission intensity ( $\mathrm{g} / \mathrm{kg}$ of milk yield) and $\mathrm{CH}_{4}$ yield when adequate grazing management was adopted $\left(\mathrm{LI}_{95 \%}\right.$ pregrazing target), while Wims et al. (2010) and Muñoz et al. (2016) reported reductions of $10 \%$ for temperate swards. It is worth mentioning that $\mathrm{CH}_{4}$ emission intensity of $\mathrm{LI}_{95} \%$ was $16.8 \mathrm{~g}$ of $\mathrm{CH}_{4} / \mathrm{kg}$ of milk yield, lower than results reported in temperate pastures (18.8 $\mathrm{g}$ of $\mathrm{CH}_{4} / \mathrm{kg}$ of milk yield), and the methane yield of $\mathrm{LI}_{95 \%}$ was similar to results obtained on temperature pastures (20.2 vs. $21.5 \mathrm{~g}$ of $\mathrm{CH}_{4} / \mathrm{kg}$ of DMI, values from Wims et al., 2010; Enriquez-Hidalgo et al., 2014; Muñoz et al., 2016). These results highlight the need to review the historical concept of tropical grasses having low herbage quality when managed under tight sward monitoring (Stobbs, 1975; Sollenberger and Burns, 2001).

Milk yield outputs per hectare increased between 40 and $50 \%$ by simply changing the pregrazing SSH of elephant grass from $\approx 135 \mathrm{~cm}$ to $100 \mathrm{~cm}$. Greater milk productivity was achieved by increased stocking rate $(+33 \%)$ and milk yield per cow $(+15 \%)$ when $\mathrm{LI}_{95 \%}$ was adopted. Greater milk yields in grazing dairy farms have been usually associated with the provision of additional feed (i.e. increased nitrogen rates onto pastures, external supplementary feed inputs; Ramsbottom et al., 2015; Macdonald et al., 2017). However, in addition to economic investments, both techniques are associated with environmental issues, such as increased GHG emissions, water and land degradation (Foley et al., 2011; Vogeler et al., 2013; Foote et al., 2015). Enteric $\mathrm{CH}_{4}$ emitted per hectare was greater for lower pre-grazing SSH and it was a function of the higher stocking rates resulting from this grazing strategy. However, this result has a small relative importance when milk yield per hectare is considered. The $51 \%$ increase in milk productivity overcame the $29 \%$ increase in enteric $\mathrm{CH}_{4}$ emissions per hectare for the $\mathrm{LI}_{95 \%}$ grazing management, and thereby determined a $16 \%$ mitigation of the main greenhouse gas from pasture-based dairy systems (Crosson et al., 2011; Guerci et al., 2013; Aguirre-Villegas et al., 2017).

In a context where the growing demand for food must be achieved through low environmental footprint practices, our findings highlighted an opportunity to improve the efficiency of tropical pasture-based dairy systems through optimization of ecological processes. Strategic grazing allows for intensification that is not coupled with increase in external resources (i.e. fertilizer, external supplements) but rather to efficient use of existing resources (i.e. solar radiation, rainwater, pasture, fertilizer, supplement). In addition, strategic grazing management is a non cost and readily available practice with easy adoption that enhances profitability of tropical pasture-based systems. The adoption of strategic grazing in tropical pasture-based systems provides the opportunity to either increase farms' total production, or use the extra land for food or forestry (further mitigating GHG emissions). The decision regarding land use depends on governmental policy or market trends. 


\subsection{Conclusions}

Strategic grazing management that reduces stem elongation in tropical forage grass swards optimizes the processes inherent to plant growth (i.e. leaf accumulation and herbage nutritive value), to plant animal interface (i.e. grazing efficiency and DMI), and animal (i.e. $\mathrm{CH}_{4}$ emission intensity and $\mathrm{CH}_{4}$ yield), resulting in greater milk yield from the same area of land. Monitoring of SSH in tropical pastures is a useful and reliable field tool that translates ecophysiological plant responses in a practical manner to farmers towards sustainable intensification at pasture-based systems in the tropics.

\section{References}

Aguirre-Villegas, H.A., Passos-Fonseca, T.H., Reinemann, D.J., Larson, R.A., 2017. Grazing intensity affects the environmental impact of dairy systems. J. Dairy Sci. 100: 6804-6821. https://doi.org/10.3168/jds.201612325.

Alexandratos, N., Bruinsma, J., 2012. World Agriculture Towards 2030/2050. FAO, Rome http://www.fao.org/fileadmin/templates/esa/Global_persepctives/world_ag_2030_50_2012_rev.pd f.

Allen, M.S., 1996. Physical constraints on voluntary intake of forages by ruminants. J. Anim. Sci. 74:3063-3075. https://www.ncbi.nlm.nih.gov/pubmed/8994921.

Allen, M.S., 2000. Effects of diet on short-term regulation of feed intake by lactating dairy cattle. J. Dairy Sci. 83:1598-1624. https://doi.org/10.3168/jds.S0022-0302(00)75030-2.

AOAC International (Ed.), 2005. Official Methods of Analysis, 18th ed. AOAC International, Gaithersburg, MD.

Barbosa, R.A., Nascimento Jr., D., Euclides, V.P.B., Da Silva, S.C., Zimmer, A.H., Torres Jr., R.A.A., 2007. Capim Tanzânia submetido a combinações entre intensidade e frequência de pastejo. Pesq. Agropec. Bras. 42:329-340. https://doi.org/10.1590/S0100-204X2007000300005.

Beukes, P.C., Scarsbrook, M.R., Gregorini, P., Romera, A.J., Clark, D.A., Catto, W., 2012. The relationship between milk production and farm-gate nitrogen surplus for the Waikato region, New Zealand. J. Environ. Manag. 93:44-51. https://doi.org/10.1016/j.jenvman.2011.08.013.

Boland, T.M., Quinlan, C., Pierce, K.M., Lynch, M.B., Kelly, A.K., Purcell, P.J., 2013. The effect of pasture pre grazing vegetation mass on methane emissions, ruminal fermentation, and average daily gain of grazing beef heifers. J. Anim. Sci. 91:3867-3874. https://doi.org/10.2527/jas2013$\underline{5900 .}$.

Brougham, R.W., 1955. A study in rate of pasture growth. Aust. J. Agric. Res. 6:804-812. https://doi.org/10.1071/AR9550804.

Brougham, R.W., 1958. Interception of light by the foliage of pure and mixed stands of pasture plants. Aust. J. Agric. Res. 9:39-52. https://doi.org/10.1071/AR9580039. 
Carnevalli, R.A., Da Silva, S.C., Bueno, A.A.O., Uebele, M.C., Bueno, F.O., Hodgson, J., Silva, G.N., Morais, J.P.G., 2006. Herbage production and grazing losses in Panicum maximum cv. Mombaça under four grazing management. Trop. Grassl.-Forrajes Trop. 40:165-176. http://tropicalgrasslands.info/public/journals/4/Historic/Tropical\%20Grasslands\%20Journal\%20ar chive/PDFs/Vol_40_2006/Vol_40_03_2006_pp165_176.pdf.

Carvalho, P.C.F., 2013. Harry Stobbs memorial lecture: can grazing behavior support innovations in grassland management? Trop. Grassl.-Forrajes Trop. 1:137-155. https://doi.org/10.17138/TGFT(1)137-155.

CEPAGRI, 2012. Centro de pesquisas meteorológicas e climáticas aplicadas à agricultura. [Center of Applied Climatic and Meteorological Research in Agriculture]. UNICAMP, Campinas. http://www.cpa.unicamp.br/outras-informacoes/clima muni 436.html.

Coleman, S.W., Moore, J.E., 2003. Feed quality and animal performance. Field Crops Res. 84:17-29. https://doi.org/10.1016/S0378-4290(03)00138-2.

Conforti, P., 2011. Looking Ahead in World Food and Agriculture: Perspectives to 2050. Food and Agriculture Organization, Rome. http://www.fao.org/docrep/014/i2280e/i2280e.pdf.

Crosson, P., Shalloo, L., O'Brien, D., Lanigan, G.J., Foley, P.A., Boland, T.M., Kenny, D.A., 2011. A review of whole farm systems models of greenhouse gas emissions from beef and dairy cattle production systems. Anim. Feed Sci. Technol. 166-167:29-45. https://doi.org/10.1016/j.anifeedsci.2011.04.001.

Da Silva, S.C., Carvalho, P.C.F., 2005. Foraging behaviour and herbage intake in the favourable tropics/subtropics. In: McGilloway, D.A. (Ed.), Grassland: A Global Resource. Wageningen Academic Publishers, Wageningen, The Netherlands :pp. 81-96. http://citeseerx.ist.psu.edu/viewdoc/download?doi=10.1.1.511.4117\&rep=rep1\&type=pdf.

Da Silva, S.C., Bueno, A.A.O., Carnevalli, R.A., Uebele, M.C., Bueno, F.O., Hodgson, J.,Matthew, C., Arnold, J.C., Morais, J.P.G., 2009. Sward structural characteristics and herbage accumulation of Panicum maximum cv. Mombaça subject to rotational stocking managements. Sci. Agric. 66:819. https://doi.org/10.1590/S010390162009000100002.

Da Silva, S.C., Sbrissia, A.F., Pereira, L.E.T., 2015. Ecophysiology of C4 forage grassesunderstanding plant growth for optimising their use and management. Agriculture 5:598-625. https://doi.org/10.3390/agriculture5030598.

Da Silva, S.C., Chiavegato, M.B., Pena, K.S., Silveira, M.C.T., Barbero, L.M., Junior, S.J.S., Rodrigues, C.S., Limão, V.A., Pereira, L.E.T., 2017. Tillering dynamics of Mulato grass subjected to strategies of rotational grazing management. J. Agric. Sci. 155:1082-1092. https://doi.org/10.1017/S0021859617000223.

Danes, M.A., Chagas, L.J., Pedroso, A.M., Santos, F.A.P., 2013. Effect of protein supplementation on milk production and metabolism of dairy cows grazing tropical grass. J. Dairy Sci. 96:407-419. https://doi.org/10.3168/jds.2012-5607. 
De Souza, J., Batistel, F., Welter, K.C., Silva, M.M.V., Costa, D.F., Santos, F.A.P., 2015. Evaluation of external markers to estimate fecal excretion, intake and digestibility in dairy cows. Trop. Anim. Health Prod. 47:265-268. https://doi.org/10.1007/s11250-014-0674-6.

Edmonson, A.J., Lean, I.J., Weaver, L.D., Farver, T.,Webster, G., 1989. A body condition scoring chart for Holstein dairy cows. J. Dairy Sci. 72:68-78. https://doi.org/10.3168/jds.S00220302(89)79081-0.

Enriquez-Hidalgo, D., Gilliland, T., Deighton, M.H., O'Donovan, M., Hennessy, D., 2014. Milk production and enteric methane emissions by dairy cows grazing fertilized perennial ryegrass pasture with or without inclusion of white clover. J. Dairy Sci. 97:1400-1412. https://doi.org/10.3168/jds.2013-7034.

Foley, J.J.A., Ramankutty, N., Brauman, K.A., Cassidy, E.S., Gerber, J.S., Johnston, M.,Mueller, N.D., O'Connell, C., Ray, D.K.,West, P.C., Balzer, C., Bennett, E.M., Carpenter, S.R., Hill, J., Monfreda, C., Polasky, S., Rockström, J., Sheehan, J., Siebert, S., Tilman, D., Zaks, D.P.M., 2011. Solutions for a cultivated planet. Nature 478:337-342. https://doi.org/10.1038/nature10452.

Fonseca, L., Mezzalira, J.C., Bremm, C., Filho, R.S.A., Gonda, H.L., Carvalho, P.C.F., 2012. Management targets formaximising the short-term herbage intake rate of cattle grazing in Sorghum bicolor. Livest. Sci. 145:205-211. https://doi.org/10.1016/j.livsci.2012.02.003.

Fonseca, L., Carvalho, P.C.F., Mezzalira, J.C., Bremm, C., Galli, J.R., Gregorini, P., 2013. Effect of sward surface height and level of herbage depletion on bite features of cattle grazing Sorghum bicolor swards. J. Anim. Sci. 91:4357-4365. https://doi.org/10.2527/jas.2012-5602.

Foote, K.J., Joy, M.K., Death, R.G., 2015. New Zealand dairy farming: milking our environment for all its worth. Environ. Manag. 56:709-720. https://doi.org/10.1007/s00267-015-0517-x.

Forbes, J., 2007. Voluntary Food Intake and Diet Selection in Farm Animals. 2nd ed. CAB International, Wallingford, UK 453p. https://doi.org/10.1079/9781845932794.0000.

Garnett, T., Godfray, H.C.J., 2012. Sustainable Intensification in Agriculture: Navigating a Course through Competing Food System Priorities. Food Climate Research Network and the Oxford Martin Programme on the Future of Food. University of Oxford, Oxford. https://www.fcrn.org.uk/sites/default/files/SI report final.pdf.

Gastal, F., Durand, J.L., 2000. Effects of nitrogen and water supply on N and C fluxes and partitioning in defoliated swards. In: Lemaire, G., Hodgson, J., de Moraes, A., Nabinger, C., de Faccio Carvalho, P.C. (Eds.), Grassland Ecophysiology and Grazing Ecology. CAB International, Wallingford, UK :pp. 15-29. https://www.cabi.org/cabebooks/ebook/20003019241.

Gimenes, F.M.A., Da Silva, S.C., Fialho, C.A., Gomes, M.B., Berndt, A., Gerdes, L., Colozza, M.T., 2011. Ganho de peso e produtividade animal em capim-marandu sob pastejo rotativo e adubação nitrogenada. Pesq. Agropec. Bras. 46:751-759. https://doi.org/10.1590/S0100204X2011000700011. 
Godfray, H., Beddington, J.R., Crute, I.R., Haddad, L., Lawrence, D., Muir, J.F., Pretty, J., Robinson, S., Thomas, S.M., Toulmin, C., 2010. Food security: the challenge of feeding 9 billion people. Science 327:812-818. https://doi.org/10.1126/science.1185383.

Goeser, J.P., Combs, D.K., 2009. An alternative method to assess 24-h ruminal in vitro neutral detergent fiber digestibility. J. Dairy Sci. 92:3833-3841. https://doi.org/10.3168/jds.2008-1136.

Gregorini, P., Gunter, S.A., Bowman,M.T., Caldwell, J.D., Masino, C.A., Coblentz,W.K., Beck, P.A., 2011. Effect of herbage depletion on short-term foraging dynamics and diet quality of steers grazing wheat pastures. J. Anim. Sci. 89:3824-3830. https://doi.org/10.2527/jas.2010-3725.

Gregorini, P., Villalba, J.J., Chilibroste, P., Provenza, F.D., 2017. Grazing management: setting the table, designing the menu and influencing the diner. Anim. Prod. Sci. 57 (7):1248-1268. https://doi.org/10.1071/AN16637.

Guerci, M., Knudsen, M.T., Bava, L., Zucali, M., Schonbach, P., Kristensen, T., 2013. Parameters affecting the environmental impact of a range of dairy farming systems in Denmark, Germany and Italy. J. Clean. Prod. 54:133-141. https://doi.org/10.1016/j.jclepro.2013.04.035.

Janssen, P.H., 2010. Influence of hydrogen on rumen methane formation and fermentation balances through microbial growth kinetics and fermentation thermodynamics. Anim. Feed Sci. Technol. 160:1-22. https://doi.org/10.1016/j.anifeedsci.2010.07.002.

Johnson, K.A., Johnson, D.E., 1995. Methane emissions from cattle. J. Anim. Sci. 73:2483-2492. https://doi.org/10.2527/1995.7382483x.

Laca, E.A., Lemaire, G., 2000. Measuring sward structure. In: Mannetje, L., Jones, R.M. (Eds.), Field and Laboratory Methods for Grassland and Animal Production Research. CABI, Wallingford, UK:pp. 103-121 https://doi.org/10.1079/9780851993515.0000.

Macdonald, K.A., Penno, J.W., Lancaster, J.A.S., Bryant, A.M., Kidd, J.M., Roche, J.R., 2017. Production and economic responses to intensification of pasture-based dairy production systems. J. Dairy Sci. 100:6602-6619. https://doi.org/10.3168/jds.2016-12497.

McDowell, R.W., Houlbrooke, D.J., 2009. Management options to decrease phosphorus and sediment losses from irrigated cropland grazed by cattle and sheep. Soil Use Manag. 25:224-233. https://doi.org/10.1111/j.1475-2743.2009.00231.x.

Mertens, D.R., 1994. Regulation of forage intake. In: Fahey Jr., G.C., Collins, M., Mertens, D.R., Moser, L.E. (Eds.), Forage Quality, Evaluation, and Utilization. American Society of Agronomy, Crop Science Society of America, Soil Science Society of America, Madison, WI:pp. 450-493. https://dl.sciencesocieties.org/publications/.../foragequalityev/45.

Moore, J.E., 1994. Forage quality indices: development and application. In: Fahey Jr., G.C. (Ed.), Forage Quality, Evaluation and Utilization. ASA, CSSA/SSSA, Madison, pp. 967-998.

Moore, K.J., Jung, H.J.G., 2001. Lignin and fiber digestion. J. Range Manag. 54:420-430. https://doi.org/10.2458/azu_jrm_v54i4_moore. 
Muñoz, C., Letelier, P.A., Ungerfeld, E.M., Morales, J.M., Hube, S., Pérez-Prieto, L.A., 2016. Effects of pre grazing herbage mass in late spring on enteric methane emissions, dry matter intake, and milk production of dairy cows. J. Dairy Sci. 99:7945-7955. https://doi.org/10.3168/jds.2016$\underline{10919 .}$.

Myers, W.D., Ludden, P.A., Nayigihugu, V., Hess, B.W., 2004. Technical note: a procedure for preparation and quantitative analysis of samples for titanium dioxide. J. Anim. Sci. 82:179-193. https://pdfs.semanticscholar.org/4ad3/c1f840f0070e800998507f77c9394f24c631.pdf.

Palhano, A.L., Carvalho, P.C.F., Dittrich, J.R., Moraes, A., Da Silva, S.C.,Monteiro, A.L.G., 2007. Características do processo de ingestão de forragem por novilhas holandesas em pastagens de capim-mombaça. Rev. Bras. Zootecn. 36:1014-1021. https://doi.org/10.1590/S1516$\underline{35982007000500005 .}$.

Pereira, L.E.T., Paiva, A.J., Geremia, E.V., Da Silva, S.C., 2014. Components of herbage accumulation in elephant grass cvar Napier subjected to strategies of intermittent stocking management. J. Agric. Sci. 152:954-966. https://doi.org/10.1017/S0021859613000695.

Pereira, L.E.T., Paiva, A.J., Geremia, E.V., da Silva, S.C., 2015a. Grazing management and tussock distribution in elephant grass. Grass Forage Sci. 70:1-12. https://doi.org/10.1111/gfs.12137.

Pereira, L.E.T., Paiva, A.J., Geremia, E.V., Da Silva, S.C., 2015b. Regrowth patterns of elephant grass (Pennisetum purpureum Schum.) subjected to strategies of intermittent stocking management. Grass Forage Sci. 70:195-204. https://doi.org/10.1111/gfs.12103.

Pérez-Prieto, L., Delagarde, R., 2013. Meta-analysis of the effect of pasture allowance on pasture intake, milk production, and grazing behavior of dairy cows grazing temperate grasslands. J. Dairy Sci. 96:6671-6689. https://doi.org/10.3168/jds.2013-6964.

Poppi, D.P., Hughes, T.P., L'Huillier, P.J., 1987. Intake of pasture by grazing ruminants. In: Nicol, A.M. (Ed.), Feeding Livestock on Pasture. Occasional Publication, New Zealand Society of Animal Production, Hamilton, New Zealand, pp. 55-63.

Poppi, D.P., McLennan, S.R., Bediye, S., de Vega, A., Zorrilla-Rios, J., 1997. Forage quality: strategies for increasing nutritive value of forages. Proceedings of the 18th International Grassland Congress, Winnipeg, Manitoba, 307-322. http://www.internationalgrasslands.org/files/igc/publications/1997/iii307.pdf.

Pretty, J., Bharucha, Z.P., 2014. Sustainable intensification in agricultural systems. Ann. Bot. 114:1571-1596. https://doi.org/10.1093/aob/mcu205.

Ramsbottom, G., Horan, B., Berry, D.P., Roche, J.R., 2015. Factors associated with the financial performance of spring-calving, pasture-based dairy farms. J. Dairy Sci. 98:3526-3540. https://doi.org/10.3168/jds.20148516. 
Royal Society, 2009. Reaping the Benefits: Science and the Sustainable Intensification of Global Agriculture. The Royal Society, London. https://royalsociety.org/ /media/Royal_Society_Content/policy/publications/2009/4294967719.pd f.

Silveira, M.C.T., Da Silva, S.C., Souza Jr., S.J., Barbero, L.M., Rodrigues, C.S., Limão, V.A., Pena, K.S., Nascimento Jr., D., 2013. Herbage accumulation and grazing losses on Mulato grass subjected to strategies of rotational stocking management. Sci. Agric. 70:242-249. https://doi.org/10.1590/S0103-90162013000400004.

Sollenberger, L.E., Burns, J.C., 2001. Canopy characteristics, ingestive behaviour and herbage intake in cultivated tropical grasslands. In: Gomide, J.A., Mattos, W.R.S., Da Silva, S.C. (Eds.), Proceedings of the 19th International Grassland Congress, São Pedro, SP, Brazil:pp. 321-327. https://pdfs.semanticscholar.org/03b2/fac7a56de5c484c7757a04fb0974f1fe2af2.pdf.

Sollenberger, L.E., Vanzant, E.S., 2011. Interrelationships among forage nutritive value and quantity and individual animal performance. Crop Sci. 51:420-432. https://doi.org/10.2135/cropsci2010.07.0408.

Stobbs, T.H., 1975. Factors limiting the nutritional value of grazed tropical pastures for beef and milk $\begin{array}{llll}\text { production. } & \text { Trop. } & \text { Grassl.-Forrajes } & \text { 9:141-149. }\end{array}$ http://tropicalgrasslands.asn.au/Tropical\%20Grasslands\%20Journal\%20archive/titles\%20only/earl y\%20vol\%20pdfs/Vol\%209\%20No\%202/vol\%209\%20\%5B2\%5D\%20Paper\%209\%20Stobbs.pdf

Struik, P.C., Kuyper, T.W., 2017. Sustainable intensification in agriculture: the richer shade of green: a review. Agron. Sustain. Dev. 37 (39). https://doi.org/10.1007/s13593017-0445-7.

Trindade, J.K., Da Silva, S.C., Souza Jr, S.J., Giacomini, A.A., Zeferino, C.V., Guarda, V.D.A., Carvalho, P.C.F., 2007. Composição morfológica da forragem consumida por bovinos de corte durante o rebaixamento do capim-marandu submetido a estratégias de pastejo rotativo. Pesq. Agropec. Bras. 42:883-890. https://doi.org/10.1590/S0100204X2007000600016.

Van Soest, P.J., Robertson, J.B., Lewis, B.A., 1991. Methods for dietary fiber, neutral detergent fiber and nonstarch polysaccharides in relation to animal nutrition. J. Dairy Sci. 74:3583-3597. https://doi.org/10.3168/jds.S0022-0302(91)78551-2.

Vogeler, I., Beukes, P.C., Burgraff, V.T., 2013. Evaluation of mitigation strategies for nitrate leaching on pasture-based dairy systems. Agric. Syst. 115:21-28. https://doi.org/10.1016/j.agsy.2012.09.012.

Voltolini, T.V., Santos, F.A.P., Martinez, J.C., Imaizumi, H., Clarindo, R.L., Penati, M.A., 2010. Produção e composição do leite de vacas mantidas em pastagens de capim-elefante submetidas a duas frequências de pastejo. Rev. Bras. Zootecn. 39 (1):121-127. https://doi.org/10.1590/S151635982010000100016. 
Wade, M.H., Carvalho, P.C.F., 2000. Defoliation patterns and herbage intake in grazed pastures. In: Lemaire, G., Hodgson, J., de Moraes, A., Carvalho, P.D.F., Nabinger, C. (Eds.), Ecophysiology of Grasslands and the Ecology of Grazing. CAB Int., Oxford, UK: pp. 233-248 https://doi.org/10.1079/9780851994529.0233.

Westberg, H.H., Johnson, K.A., Cossalman,M.W.,Michal, J.J., 1998. A SF6 Tracer Technique: Methane Measurement from Ruminants. 2nd ed. Pullman-Washington, Washington State University.

Wilson, J.R., Kennedy, P.M., 1996. Plant and animal constraints to voluntary feed intake associated with fibre characteristics and particle breakdown and passage in ruminants. Aust. J. Agric. Res. 47:199-225. https://doi.org/10.1071/AR9960199.

Wims, C.M., Deighton, M.H., Lewis, E., O'Loughlin, B., Delaby, L., Boland, T.M., O'Donovan, M., 2010. Effect of pregrazing herbage mass on methane production, dry matter intake, and milk production of grazing dairy cows during the mid-season period. J. Dairy Sci. 93:4976-4985. https://doi.org/10.3168/jds.2010-3245. 


\title{
4. STRATEGIC GRAZING MANAGEMENT AND NITROUS OXIDE FLUXES FROM PASTURE SOILS IN TROPICAL DAIRY SYSTEMS
}

\begin{abstract}
Greenhouse gases emissions are considered the most important among all environmental issues of dairy farming systems. Nitrous oxide $\left(\mathrm{N}_{2} \mathrm{O}\right)$ has particular importance owing to its global warming potential and stratospheric ozone depletion. The objective of this study was to investigate the influence of two rotational grazing strategies characterized by two pre-grazing targets $(95 \%$ and maximum canopy light interception during sward regrowth; $\mathrm{LI}_{95 \%}$ and $\mathrm{LI}_{\mathrm{Max}}$, respectively) on $\mathrm{N}_{2} \mathrm{O}$ fluxes from soil and milk productivity in a tropical dairy farming system based on elephant grass (Pennisetum purpureum Schum. cv. Cameroon). The general hypothesis was that frequent defoliations generated by the $\mathrm{LI}_{95 \%}$ pre-grazing target would increase $\mathrm{N}_{2} \mathrm{O}$ fluxes, however this greater emission would ultimately be compensated by greater milk productivity. Results indicated that $\mathrm{LI}_{95 \%}$ pre-grazing target provided more frequent defoliation than $\mathrm{LI}_{\mathrm{Max}}$. Water-filled pore space (WFPS), soil and chamber temperatures were affected by sampling period $\left(\mathrm{P}_{1}\right.$ and $\left.\mathrm{P}_{2}\right)$. There was significant treatment $x$ sampling period interaction on soil $\mathrm{NH}_{4}{ }^{+}$concentration, and it was most likely associated with urinary$\mathrm{N}$ discharge. During $\mathrm{P}_{1}$, there was a greater urinary-N discharge for $\mathrm{LI}_{95 \%}$ than $\mathrm{LI}_{\mathrm{Max}}(26.3 \mathrm{vs} .20 .9 \mathrm{~kg}$ of urinary-N/paddock) caused by higher stocking rate (10.0 vs. $8.3 \mathrm{cows} / \mathrm{ha})$, which resulted in greater $\mathrm{N}_{2} \mathrm{O}$ fluxes for $\mathrm{LI}_{95 \%}$. Inversely, during $\mathrm{P}_{2}$, the soil $\mathrm{NH}_{4}{ }^{+}$and $\mathrm{N}_{2} \mathrm{O}$ fluxes were greater for $\mathrm{LI}_{\text {Max }}$ than $\mathrm{LI}_{95 \%}$. During this period, the greater urinary-N discharge (46.8 vs. $44.8 \mathrm{~kg}$ of urinary-N/paddock) was likely associated with greater stocking period (1.88 vs. 1.46 days) for $\mathrm{LI}_{\mathrm{Max}}$ relative to $\mathrm{LI}_{95 \%}$, since both treatments had similar stocking rate (9.5 vs. 9.9 cows/ha). Converting hourly $\mathrm{N}_{2} \mathrm{O}$ fluxes to daily basis and relating to milk productivity, $\mathrm{LI}_{95 \%}$ was $35 \%$ more efficient than $\mathrm{LI}_{\mathrm{Max}}\left(0.36\right.$ vs. $0.55 \mathrm{~g} \mathrm{~N}-\mathrm{N}_{2} \mathrm{O} / \mathrm{kg}$ milk.ha.day). In addition, $\mathrm{LI}_{95 \%}$ pre-grazing target decreased $34 \%$ urea-N applied per milk yield per hectare ( 0.57 vs. $0.86 \mathrm{~g}$ urea-N/kg milk.ha.day). Strategic grazing management represented by the $\mathrm{LI}_{95 \%}$ pre-grazing target allows for intensification of tropical pasture-based dairy systems enhancing milk productivity and decreasing $\mathrm{N}-\mathrm{N}_{2} \mathrm{O}$ emitted per kg of milk.
\end{abstract}

Keywords: Canopy light interception; Nitrous oxide fluxes; Grazed soils; Soil nitrogen; Land-use improvement; Elephant grass

\subsection{Introduction}

Greenhouse gases (GHG) emissions are considered the most important among all environmental issues of dairy farming systems (O'Brien et al., 2012; Guerci et al., 2013; Gregorini et al, 2016). Nitrous oxide $\left(\mathrm{N}_{2} \mathrm{O}\right)$ has particular importance owing to its global warming potential (265298 times greater than carbon dioxide; Myhre et al., 2013) and stratospheric ozone depletion (Ravishankara et al., 2009; IPCC, 2014). It is the main GHG from the soil and the second most representative among all GHG, ranging from 15 (housed) to $25 \%$ (pasture-based) of total GHG emissions in dairy farming systems (Aguirre-Villegas et al., 2017). Methane $\left(\mathrm{CH}_{4}\right)$ and carbon dioxide $\left(\mathrm{CO}_{2}\right)$ from soils are proportionally less important than $\mathrm{N}_{2} \mathrm{O}$ in dairy farming systems (Jarvis et al., 1995; de Klein et al., 2008; Aguirre-Villegas et al., 2017). 
Nitrous oxide is formed through microbial transformation of nitrogen $(\mathrm{N})$ compounds into the soil, typically by incomplete denitrification or by nitrification (Wrage et al., 2001; Saggar et al., 2013). Nitrous oxide fluxes are affected by a wide range of proximal and distal regulators, making its regulation a very complex process (de Klein et al., 2008; Luo et al., 2017). Proximal soil factors include mineral nitrogen $\left(\mathrm{NH}_{4}{ }^{+}\right.$and $\left.\mathrm{NO}_{3}{ }^{-}\right)$and organic carbon availabilities, moisture, $\mathrm{pH}$, temperature, and texture that, in turn, are affected by distal regulators such as rainfall or irrigation, soil compaction, organic matter and $\mathrm{N}$ inputs (de Klein et al., 2008; Luo et al., 2017). Periods when soil characteristics favorable to $\mathrm{N}_{2} \mathrm{O}$ production coincide are called "hot moments" (Luo et al., 2017). In tropical conditions, these "hot moments" usually occur during spring and summer when pastures are intensively growing owing to the abundance of solar radiation, rainfall, and $\mathrm{N}$ inputs.

Grazing management strategies can strongly affect the majority of distal regulators. It determines ecophysiological plant processes such as herbage growth, senescence and decay (Da Silva et al., 2009; Pereira et al., 2014; Pereira et al., 2015; Da Silva et al., 2015; Congio et al., 2018) that strongly affect animal responses such as herbage intake (Congio et al., 2018), herbage losses by cattle trampling (Carnevalli et al., 2006; Silveira et al., 2013; Congio et al., 2018), stocking rate (Voltolini et al., 2010; Gimenes et al., 2011; Congio et al., 2018), excreta spatial distribution (White et al., 2001; Auerswald et al., 2010), and $\mathrm{N}$ load into pastures (Vibart et al., 2017). These factors, in turn, modify soil properties (i.e. bulk density, moisture, temperature, $\mathrm{pH}$, aeration) (Warren et al., 1986; Silva et al., 2003; Schmalz et al., 2013) that affect microbial community growth and activity (Bardgett et al., 1996; Bardgett et al., 2001; Bardgett and Wardle, 2003) determining the intensity of processes associated to $\mathrm{N}_{2} \mathrm{O}$ fluxes from soils (de Klein et al., 2008; Levine et al., 2011; Luo et al., 2017).

The majority of studies involving $\mathrm{N}_{2} \mathrm{O}$ flux from pasture soils have been addressed to assess the effects of proximal factors on processes and emission factors in temperate conditions (Saggar et al., 2013; de Klein et al., 2014; Barneze et al., 2015; Venterea et al., 2015; Gardiner et al., 2016; Samad et al., 2016; Clough et al., 2017; Gardiner et al., 2017; van der Weerden et al. 2017; Luo et al., 2018; Rex et al., 2018). The little information available for tropical pastures has also focused on nitrous oxide fluxes related to proximal factors within urine patches (Barneze et al., 2014; Lessa et al., 2014; Mazzetto et al., 2014; Mazzetto et al., 2015). There is no information available regarding $\mathrm{N}_{2} \mathrm{O}$ fluxes from soils of tropical pasture-based dairy farming systems, as influenced by grazing management strategies. In fact, farming scale studies are scarce even in temperate conditions. Experimental approaches have shown that intensively managed grasslands are stronger sources of $\mathrm{N}_{2} \mathrm{O}$ than extensively managed grasslands owing to greater inputs of $\mathrm{N}$ fertilizer and excreta (Smith et al., 2001; Flechard et al., 2007; Rafique et al., 2011). However, they have not accounted for animal outputs that are usually greater in intensively managed systems and could compensate the higher $\mathrm{N}_{2} \mathrm{O}$ fluxes. The objective of this study was to investigate the influence of two rotational grazing strategies characterized by two pre-grazing targets (95\% and maximum canopy light interception during sward regrowth; $\mathrm{LI}_{95 \%}$ and $\mathrm{LI}_{\mathrm{Max}}$, respectively) on $\mathrm{N}_{2} \mathrm{O}$ fluxes from soil and milk productivity in a tropical 
dairy farming system based on elephant grass (Pennisetum purpureum Schum. cv. Cameroon). The general hypothesis was that frequent defoliations generated by the $\mathrm{LI}_{95} \%$ pre-grazing target would increase $\mathrm{N}_{2} \mathrm{O}$ fluxes, however this greater emission would ultimately be compensated by greater milk productivity.

\subsection{Material and Methods}

All procedures for this study were approved by the Animal (15.5.1246.11.2) and Environment Ethics Committees (17.5.999.11.9) at the University of São Paulo, College of Agriculture "Luiz de Queiroz" (USP/ESALQ).

\subsubsection{Study site}

The experiment was conducted in Piracicaba, SP, Brazil $\left(22^{\circ} 42^{\prime} \mathrm{S}, 47^{\circ} 38^{\prime} \mathrm{W}\right.$ and 546 a.s.l.) on a rainfed, non-irrigated elephant grass (Pennisetum purpureum Schum. cv. Cameroon) pasture established in 1972 in a high fertility Eutroferric Red Nitossol (Pereira et al., 2014). The climate is sub-tropical with dry winters and $1328 \mathrm{~mm}$ average annual rainfall (CEPAGRI, 2012). The lowest and highest mean temperatures were recorded in July $\left(19.7^{\circ} \mathrm{C}\right)$ and December $\left(27.1^{\circ} \mathrm{C}\right)$, respectively. The greatest accumulated rainfall was observed from late spring to summer $(1090 \mathrm{~mm}$ from November 2015 to March 2016), and the lowest from winter to early spring (356 mm from June to October 2015). Soil properties $(0-10 \mathrm{~cm})$ at the beginning of each sampling period are presented in Table 1.

Table 1. Soil properties $(0-10 \mathrm{~cm})$ at the beginning of each sampling period (P1 and P2)

\begin{tabular}{|c|c|c|c|c|c|c|c|c|}
\hline & Clay & Sand & Silt & Bulk Density & $\mathbf{p H}$ & OM & $\mathrm{NH}_{4}{ }^{+}$ & $\mathrm{NO}_{3}{ }^{-}$ \\
\hline & & $\mathrm{g} / \mathrm{kg}$ & & $\mathrm{g} / \mathrm{cm}^{3}$ & $\mathrm{CaCl}_{2}$ & $\mathrm{~g} / \mathrm{dm}^{3}$ & $\mathrm{mg} / \mathrm{kg} \mathrm{d}$ & dry soil \\
\hline & \multicolumn{8}{|c|}{$\mathbf{P}_{1}^{*}$} \\
\hline $\mathrm{LI}_{95 \%}$ & 502 & 168 & 330 & 1.31 & 5.1 & 43 & 283.4 & 5.0 \\
\hline \multirow[t]{2}{*}{$\mathrm{LI}_{\text {Max }}$} & 478 & 172 & 350 & 1.30 & 5.0 & 46 & 113.1 & 8.8 \\
\hline & \multicolumn{8}{|c|}{$\mathbf{P}_{2}^{* * *}$} \\
\hline $\mathrm{LI}_{95 \%}$ & 511 & 179 & 310 & 1.32 & 5.1 & 43 & 76.6 & 20.6 \\
\hline$L_{\text {Max }}$ & 487 & 193 & 320 & 1.44 & 5.1 & 53 & 318.4 & 1.4 \\
\hline
\end{tabular}

\subsubsection{Treatments and experimental design}

The two treatments were pre-grazing targets of either $95 \%$ or maximum canopy light interception during regrowth $\left(\mathrm{LI}_{95 \%}\right.$ and $\mathrm{LI}_{\mathrm{Max}}$, respectively). The 2.5 ha experimental area was divided into two farmlets of 18 paddocks ( $686 \mathrm{~m}^{2}$ on average) each, according to a randomized complete block 
design, with six replications. The slope and chemical soil characteristics were considered as blocking criteria.

Treatments based on canopy light interception resulted in contrastant sward structures and determined pre-grazing sward surface heights $(\mathrm{SSH})$ of $100 \mathrm{~cm}\left(\mathrm{LI}_{95} \%\right.$ and $135 \mathrm{~cm}\left(\mathrm{LI}_{\mathrm{Max}}\right)$. For both pre-grazing SSH, the herbage depletion level (post-grazing height) corresponded to $50 \%$ of the pregrazing SSH as a means to maintain high short-term rates of herbage intake (Fonseca et al., 2012; Carvalho, 2013). Treatments were allocated to the farmlets in mid-January 2015 after grazing and mowing at $45-\mathrm{cm}$ for standardization. During 11-months prior to field measurements, each farmlet was adapted to its respective grazing management strategy. Paddocks were rotationally grazed by $10-$ 13 dairy cows in order to keep grazing management targets. The adaptation period was necessary to adapt sward structure to treatments and to identify the corresponding pre-grazing SSH for the LI pregrazing targets used ( $\mathrm{LI}_{95 \%}$ and $\mathrm{LI}_{\mathrm{Max}}$ ) (Congio et al., 2018).

Measurements were performed after the adaptation period during the second rainy season from December 2015 to April 2016 (experimental period). A total of $215 \mathrm{~kg} \mathrm{~N} / \mathrm{ha}$ (as urea, 45\% of N) was applied throughout the experimental period. Because grazing interval was not constant (as a consequence of experimental design), the total amount of $\mathrm{N}$ to be applied was divided throughout the experimental period (119 days) and a daily rate of $\mathrm{N}$ fertilizer was calculated. The amount of $\mathrm{N}$ applied per paddock after each grazing was proportional to the length of the corresponding rest period (daily rate $\times$ rest period), ensuring similar $\mathrm{N}$ fertilizer application to both treatments at the end of the experimental period (Da Silva et al., 2017).

\subsubsection{Soil flux measurements, analysis and flux calculation}

Soil gaseous fluxes were measured using the non-ventilated closed static chamber methodology updated by the Global Research Alliance on Agricultural Greenhouse Gases (de Klein and Harvey, 2015). Gas samples were collected during two sampling periods throughout the experimental period $\left(\mathrm{P}_{1}=01 / 08 / 2016\right.$ to $01 / 22 / 2016$ and $\mathrm{P}_{2}=02 / 25 / 2016$ to $03 / 10 / 2016$ ). Measurements were made at post-grazing, immediately after $\mathrm{N}$ fertilization with ten chambers randomly placed 5-cm into bare ground in each paddock.

Chambers of $17.67 \mathrm{~L}$ were made of PVC, composed of a base $(30 \mathrm{~cm}$ diameter and $20 \mathrm{~cm}$ height) plus cap (30 cm diameter and $10 \mathrm{~cm}$ height), and were insulated with thermal blanket to avoid heating during sampling (de Klein et al., 2014; Di et al., 2016). Gas samples were collected immediately after chamber closing, and at 30 and $60 \mathrm{~min}$. Samples were collected from cap sampling port using $20 \mathrm{~mL}$ plastic syringes (Becton Dickinson, Franklin Lakes, NJ, EUA) and precision glide needles $(0.8 \times 40 \mathrm{~mm}$; BD), and injected into sealed and evacuated $10 \mathrm{~mL}$ glass sample vials. Gas sampling started $24 \mathrm{~h}$ after chamber placement to allow soil microbial community to stabilize and 
minimize overestimation or underestimation of emissions (Chiavegato et al., 2015). Samples were performed during five consecutive days, and then every five days until the $15^{\text {th }}$ day after fertilization. Chambers were removed after $\mathrm{P}_{1}$ evaluation and re-placed at the beginning of $\mathrm{P}_{2}$. All samples were collected from 8 to 9:15 am (Alves et al., 2012) and analyzed using gas chromatography at the Laboratory of Analytical Chemistry (Embrapa Pecuária Sudeste, São Carlos, SP, BRA).

The chromatograph GC-2014 (Shimadzu, Columbia, MD, EUA) was equipped with electron capture detectors (ECD) at $325^{\circ} \mathrm{C}$ (column HayeSep T 80/100) for $\mathrm{N}_{2} \mathrm{O}$ and flame ionization detectors (FID) at $250{ }^{\circ} \mathrm{C}$ for $\mathrm{CO}_{2}$ (column HayeSep $\mathrm{T}$ 80/100). Calibration curves were established using standard certified gases for $\mathrm{CO}_{2}(260.2 \pm 0.68 \% ; 508.3 \pm 0.61 \%, 1058 \pm 1.37 \%$ and $1995 \pm 0.54 \%$ ppm) and $\mathrm{N}_{2} \mathrm{O}(257.3 \pm 0.76 \% ; 502.8 \pm 0.69 \%, 999.5 \pm 1.77 \%$ and $2328 \pm 4.84 \%$ ppt $)$. Gas chromatography outputs were analyzed to determine linearity from 0 to $60 \mathrm{~min}$. A strong linear relationship was observed for $\mathrm{N}_{2} \mathrm{O}\left(\mathrm{r}^{2}=0.88\right)$ and the hourly gas fluxes were calculated according to the increase of gas concentration into the head space over sampling time (de Klein et al., 2014; Luo et al., 2018):

$$
\text { Gas flux }=\frac{\delta G a s}{\delta T} \times \frac{M}{V m} \times \frac{V}{A}
$$

where $\delta \mathrm{Gas}$ is the increase in head space gas concentration overtime $(\mu \mathrm{L} / \mathrm{L}) ; \delta \mathrm{T}$ is the enclosure period (hours); $\mathrm{M}$ is the molar weight of $\mathrm{N}$ in $\mathrm{N}_{2} \mathrm{O} ; \mathrm{Vm}$ is the molar volume of gas at the sampling temperature $(\mathrm{L} / \mathrm{mol}) ; \mathrm{V}$ is the headspace volume $\left(\mathrm{m}^{3}\right)$; and $\mathrm{A}$ is the area covered $\left(\mathrm{m}^{2}\right)$. Fluxes were corrected for chamber bias to account for suppression of the surface-atmosphere concentration gradient (Venterea, 2010) and hourly fluxes were assumed to represent mean daily fluxes (de Klein et al., 2014).

\subsubsection{Weather and ancillary measurements}

Atmospheric pressure, ambient temperature, and rainfall were daily monitored at the weather station located at $50 \mathrm{~m}$ from the experimental area. Soil and head-space temperature were recorded for each chamber in each timepoint with a digital thermometer (TE-300, Instrutherm, São Paulo, SP, BRA). At the first day of each sampling period, four cores of each paddock were collected to determine soil bulk and soil particle densities. During the first day of sampling, additional soil samples were taken at $5-\mathrm{cm}$ depth adjacent to each chamber in order to determine soil nitrate $\left(\mathrm{NO}_{3}{ }^{-}\right)$and ammonium $\left(\mathrm{NH}_{4}{ }^{+}\right)$. Soil $\mathrm{N}$ was extracted for one hour with $2 \mathrm{M} \mathrm{KCl}$, filtered (Whatman 42) and samples were analyzed for mineral $\mathrm{N}$ concentration by flow injection analysis (ASIA; Ismatec, Zürich, Switzerland). At each sampling day prior to gas collection, soil samples were taken $(0-5 \mathrm{~cm})$ from adjacent area of each chamber for soil gravimetric moisture determination $\left(24 \mathrm{~h}\right.$ at $\left.105{ }^{\circ} \mathrm{C}\right)$. Volumetric water contents were calculated by multiplying gravimetric water contents by soil bulk 
density and soil water-filled pore-space (WFPS) was calculated by dividing volumetric water content by total soil porosity (de Klein et al., 2014; Luo et al., 2018).

\subsubsection{Statistical analysis}

Analysis of variance was performed using the Mixed Procedure of SAS (SAS 9.3; SAS Institute Inc., Cary, NC). Different structures of the variance-covariance matrices were tested, and variance components matrix was chosen as the best fit for the majority of variables based on the Bayesian Information Criterion. The model included fixed effects of treatment, sampling period, and their interaction, and random effect of chamber. Chambers were considered experimental units and sampling periods were treated as repeated measures. Soil temperature, air temperature, WFPS, soil $\mathrm{NH}_{4}{ }^{+}$and soil $\mathrm{NO}_{3}{ }^{-}$were tested as explanatory variables. Means were calculated using the LSMEANS statement, compared using the Student's t-test and differences were declared significant at $P \leq 0.05$. For $\mathrm{N}_{2} \mathrm{O}$ fluxes, WFPS was used as covariate. To better understand the relations among dependent variables, a principal component analysis (PCA) was performed using a data set comprised of $\mathrm{N}_{2} \mathrm{O}$ fluxes, soil $\mathrm{NH}_{4}{ }^{+}$, soil $\mathrm{NO}_{3}{ }^{-}$, soil temperature, chamber temperature, and WFPS. Principal components scores were submitted to ANOVA to describe and interpret the effects of treatment and periods (Jolliffe, 2002).

\subsection{Results}

\subsubsection{Weather conditions}

Weather conditions during the two sampling periods are presented in Figure 1. Air temperature ranged from 16.6 to $35.2{ }^{\circ} \mathrm{C}$ with average of $25.7^{\circ} \mathrm{C}$ during $\mathrm{P}_{1}$ (Figure 1A). Similarly, during $\mathrm{P}_{2}$, air temperature ranged from 18.4 to $33.3{ }^{\circ} \mathrm{C}$ with average of $24.9{ }^{\circ} \mathrm{C}$ (Figure 1B). Average soil temperatures were 22.7 and $24.7^{\circ} \mathrm{C}$ for $\mathrm{P}_{1}$ and $\mathrm{P}_{2}$, respectively. Accumulated rainfall was $199 \mathrm{~mm}$ during $\mathrm{P}_{1}$ and 106 during $\mathrm{P}_{2}$ (Figures $1 \mathrm{~A}$ and $1 \mathrm{~B}$, respectively). 


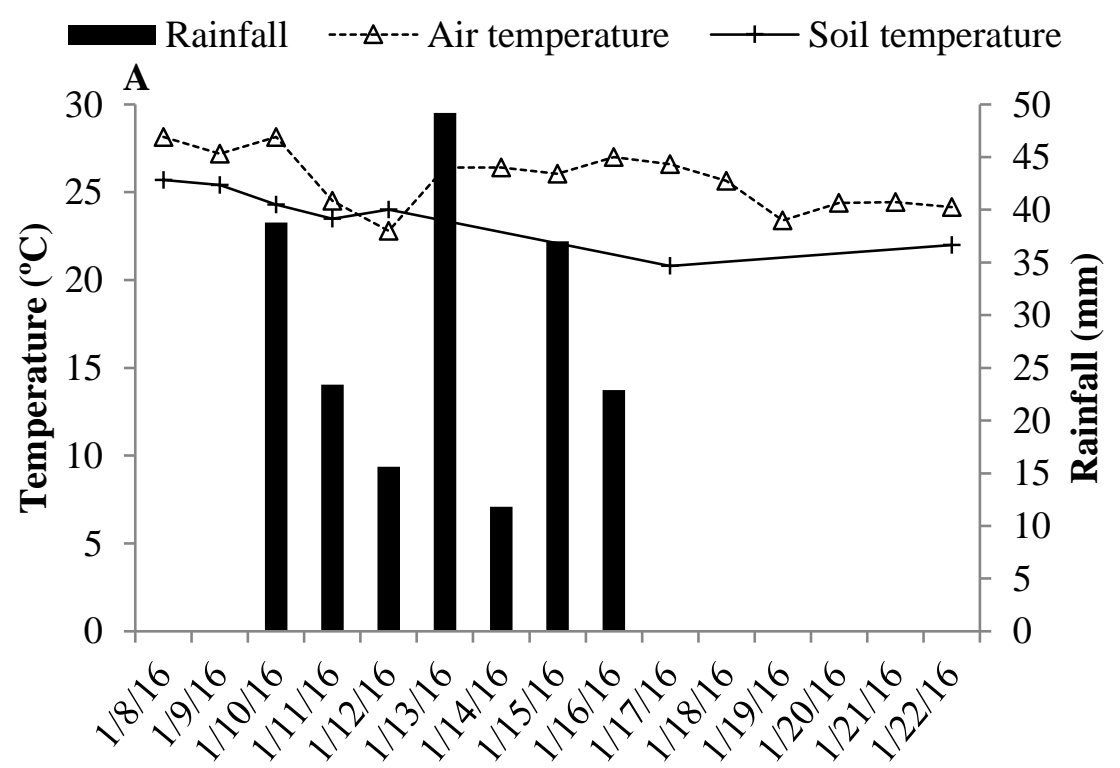

Days

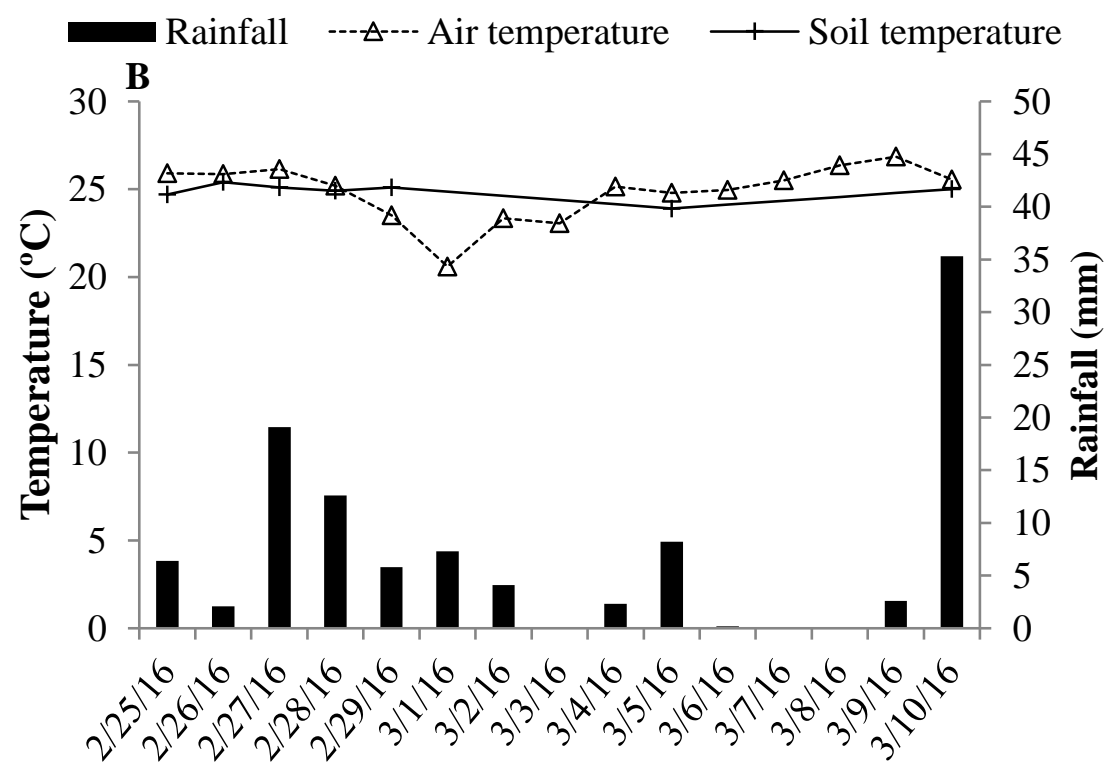

Days

Figure 1. Air and soil $(0-5 \mathrm{~cm})$ temperatures $\left({ }^{\circ} \mathrm{C}\right)$ and rainfall $(\mathrm{mm})$ during sampling periods $\mathrm{P}_{1}(\mathrm{~A})$ and $\mathrm{P}_{2}$ (B) at the study site (Jan-Mar 2016).

\subsubsection{Soil parameters}

Water-filled pore space, soil and chamber temperatures were affected by sampling period ( $P$ $<0.01$ ) being greater for $\mathrm{P}_{2}$ than $\mathrm{P}_{1}$ (Table 2). Both soil $\mathrm{NH}_{4}{ }^{+}$and $\mathrm{NO}_{3}{ }^{-}$concentrations were not affected by treatment or sampling period $(P>0.05)$, however there was a significant interaction between treatments and sampling period for soil $\mathrm{NH}_{4}{ }^{+}(P=0.0006)$ and a trend for soil $\mathrm{NO}_{3}^{-}(P=$ 0.0725). During $\mathrm{P}_{1}$, there was an effect of LI pre-grazing targets on soil $\mathrm{NH}_{4}{ }^{+}$, with greater values observed for $\mathrm{LI}_{95 \%}$ than $\mathrm{LI}_{\text {Max }}$; however, during $\mathrm{P}_{2}$ soil $\mathrm{NH}_{4}{ }^{+}$was greater for $\mathrm{LI}_{\text {Max }}$ than $\mathrm{LI}_{95 \%}(P<$ 
0.05). Water-filled pore space and rainfall patterns for both periods are presented in Figure 2. Days with no rainfall markedly decreased WFPS during the beginning and the end of $\mathrm{P}_{1}$ (Figure 2A). There was no effect of LI pre-grazing target on WFPS during $\mathrm{P}_{1}(P=0.9967$; Figure $2 \mathrm{~A})$, but the effect was significant during $\mathrm{P}_{2}(P=0.05$; Figure $2 \mathrm{~B})$.

Table 2. Water-filled pore space (WFPS), soil temperature, chamber temperature, soil ammonium and nitrate concentrations from soil established with elephant grass subjected to strategies of rotational stocking management $\left(\mathrm{LI}_{95 \%}\right.$ or $\mathrm{LI}_{\mathrm{Max}}$ ) during sampling periods $\mathrm{P}_{1}(01 / 08 / 2016$ to $01 / 22 / 2016)$ and $\mathrm{P}_{2}(02 / 25 / 2016$ to $03 / 10 / 2016)(\mathrm{n}=10)$

\begin{tabular}{|c|c|c|c|c|c|c|}
\hline & \multicolumn{2}{|c|}{ Period } & \multirow{2}{*}{ SEM $^{1}$} & \multicolumn{3}{|c|}{$P$-value } \\
\hline & 1 & 2 & & Trt $^{2}$ & Per $^{3}$ & Trt $\times$ Per \\
\hline & \multicolumn{2}{|c|}{ WFPS, \% } & \multirow[b]{2}{*}{1.57} & \multirow[b]{2}{*}{0.1654} & \multirow[b]{2}{*}{$<0.0001$} & \multirow[b]{2}{*}{0.1672} \\
\hline $\begin{array}{l}\mathrm{LI}_{95 \%} \\
\mathrm{LI}_{\text {Max }}\end{array}$ & 77.8 & 94.5 & & & & \\
\hline & \multicolumn{2}{|c|}{ Soil Temp., ${ }^{\circ} \mathrm{C}$} & \multirow[b]{2}{*}{0.11} & \multirow[b]{2}{*}{0.4125} & \multirow[b]{2}{*}{$<0.0001$} & \multirow[b]{2}{*}{0.4631} \\
\hline $\begin{array}{l}\mathrm{LI}_{95 \%} \\
\mathrm{LI}_{\text {Max }}\end{array}$ & 23.7 & 24.9 & & & & \\
\hline \multicolumn{3}{|c|}{ Chamber Temp., ${ }^{\circ} \mathrm{C}$} & \multirow[b]{2}{*}{0.14} & \multirow[b]{2}{*}{0.7344} & \multirow[b]{2}{*}{$<0.0001$} & \multirow[b]{2}{*}{0.8221} \\
\hline $\begin{array}{l}\mathrm{LI}_{95 \%} \\
\mathrm{LI}_{\operatorname{Max}}\end{array}$ & 22.6 & 23.7 & & & & \\
\hline \multicolumn{3}{|c|}{$\mathrm{NH}_{4}{ }^{+}, \mathrm{mg} / \mathrm{kg}$ dry soil } & \multirow[b]{2}{*}{69.44} & \multirow[b]{2}{*}{0.8771} & \multirow[b]{2}{*}{0.4915} & \multirow[b]{2}{*}{0.0006} \\
\hline $\begin{array}{l}\mathrm{LI}_{95 \%} \\
\mathrm{LI}_{\text {Max }}\end{array}$ & $\begin{array}{c}283.4 \mathrm{Aa} \\
21.4 \mathrm{Bb}\end{array}$ & $\begin{array}{c}76.6 \mathrm{Bb} \\
318.4 \mathrm{Aa} \\
\end{array}$ & & & & \\
\hline \multicolumn{3}{|c|}{$\mathrm{NO}_{3}{ }^{-}, \mathrm{mg} / \mathrm{kg}$ dry soil } & \multirow[b]{2}{*}{6.18} & \multirow[b]{2}{*}{0.2218} & \multirow[b]{2}{*}{0.5126} & \multirow[b]{2}{*}{0.0725} \\
\hline $\begin{array}{l}\mathrm{LI}_{95 \%} \\
\mathrm{LI}_{\text {Max }}\end{array}$ & $\begin{array}{l}5.0 \mathrm{Aa} \\
8.8 \mathrm{Aa}\end{array}$ & $\begin{array}{c}20.6 \mathrm{Aa} \\
1.4 \mathrm{Ba}\end{array}$ & & & & \\
\hline $\begin{array}{l}\text { Means fo } \\
\text { not differ } \\
\text { Standard } \\
\text { Treatme } \\
\text { Samplin }\end{array}$ & $\begin{array}{l}\text { wed by the } s \\
>0.05 \text { ) } \\
\text { ror of the mea } \\
\text { effect } \\
\text { eriod effect }\end{array}$ & ne capital lett & & & & \\
\hline
\end{tabular}



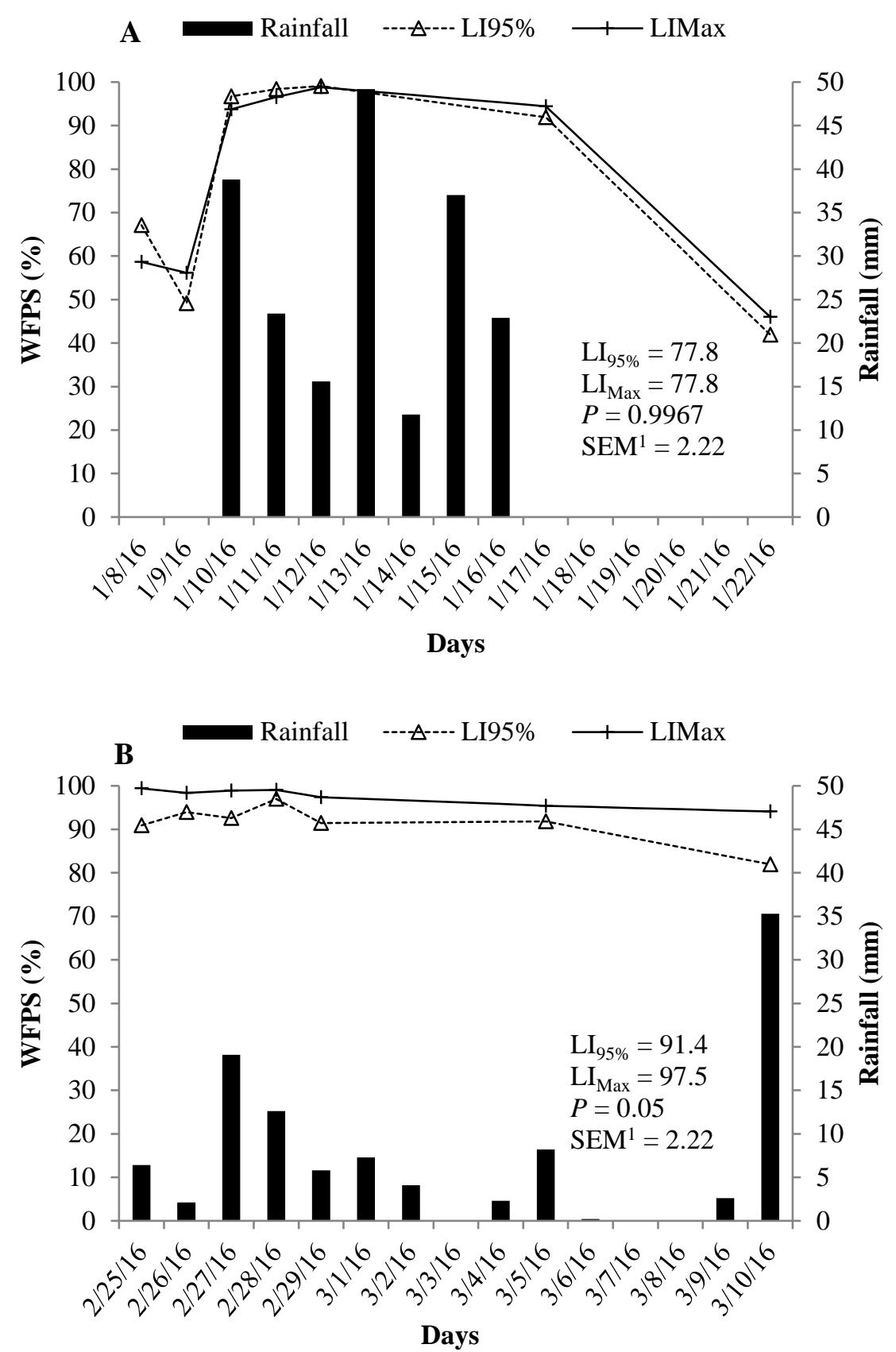

Figure 2. Water-filled pore space (WFPS) and rainfall (mm) during sampling periods $\mathrm{P}_{1}(\mathrm{~A})$ and $\mathrm{P}_{2}$ (B) at the study site (Jan-Mar 2016).

${ }^{1}$ Standard error of the mean

\subsubsection{Nitrous oxide fluxes}

Nitrous oxide fluxes were not affected by pre-grazing targets $(P=0.9975)$, however they were strongly affected by sampling period and WFPS $(P<0.01$; Table 3$)$. On average, $\mathrm{N}_{2} \mathrm{O}$ fluxes were greater during $\mathrm{P}_{1}$ than $\mathrm{P}_{2}$ (375.9 vs. $\left.134.5 \mu \mathrm{g} \mathrm{N}-\mathrm{N}_{2} \mathrm{O} / \mathrm{m}^{2} . \mathrm{h} ; P<0.01\right)$. There was a significant interaction between treatments and sampling period $(P=0.004)$. During $\mathrm{P}_{1}, \mathrm{~N}_{2} \mathrm{O}$ fluxes were greater for $\mathrm{LI}_{95 \%}(P=0.0405)$ and during $\mathrm{P}_{2}$ fluxes were greater for $\mathrm{LI}_{\mathrm{Max}}(P=0.0414)$. Nitrous oxide fluxes 
across sampling periods and days are shown in Figure 3. Across days, there were no differences on $\mathrm{N}_{2} \mathrm{O}$ fluxes during $\mathrm{P}_{1}(P>0.05$; Figure $3 \mathrm{~A})$. During $\mathrm{P}_{2}$, four of seven days had greater $\mathrm{N}_{2} \mathrm{O}$ fluxes for $\mathrm{LI}_{\text {Max }}$ than $\mathrm{LI}_{95 \%}(P<0.05$; Figure $3 \mathrm{~B})$.

Table 3. Nitrous oxide fluxes $\left(\mu \mathrm{g} \mathrm{N}-\mathrm{N}_{2} \mathrm{O} / \mathrm{m}^{2}\right.$.h) from soil established with elephant grass subjected to strategies of rotational stocking management $\left(\mathrm{LI}_{95 \%}\right.$ or $\left.\mathrm{LI}_{\mathrm{Max}}\right)$ during sampling periods $\mathrm{P}_{1}(01 / 08 / 2016$ to $01 / 22 / 2016)$ and $\mathrm{P}_{2}(02 / 25 / 2016$ to $03 / 10 / 2016)(\mathrm{n}=10)$

\begin{tabular}{|c|c|c|c|c|c|c|c|}
\hline & \multicolumn{2}{|c|}{ Period } & \multirow{2}{*}{ SEM $^{1}$} & \multicolumn{4}{|c|}{$P$-value } \\
\hline & 1 & 2 & & Trt $^{2}$ & Per $^{3}$ & Trt $\times$ Per & WFPS $^{4}$ \\
\hline $\begin{array}{l}\mathrm{LI}_{95 \%} \\
\mathrm{LI}_{\text {Max }}\end{array}$ & $\begin{array}{l}432.8 \mathrm{Aa} \\
319.1 \mathrm{Ba}\end{array}$ & $\begin{array}{c}77.6 \mathrm{Bb} \\
191.5 \mathrm{Ab}\end{array}$ & 40.61 & 0.9975 & $<0.0001$ & 0.004 & $<0.0001$ \\
\hline $\begin{array}{l}\text { Means ff } \\
\text { differ }(P \\
\text { 'Standarc } \\
\text { }{ }^{2} \text { Treatme } \\
{ }^{3} \text { Samplin } \\
{ }^{4} \text { Water-fi }\end{array}$ & $\begin{array}{l}\text { ollowed by the } \\
>0.05 \text { ) } \\
\text { d error of the } \mathrm{m} \\
\text { ent effect } \\
\text { ng period effect } \\
\text { illed pore space }\end{array}$ & $\begin{array}{l}\text { same capital } 1 \\
\text { ean } \\
\text { effect }\end{array}$ & & & & & \\
\hline
\end{tabular}




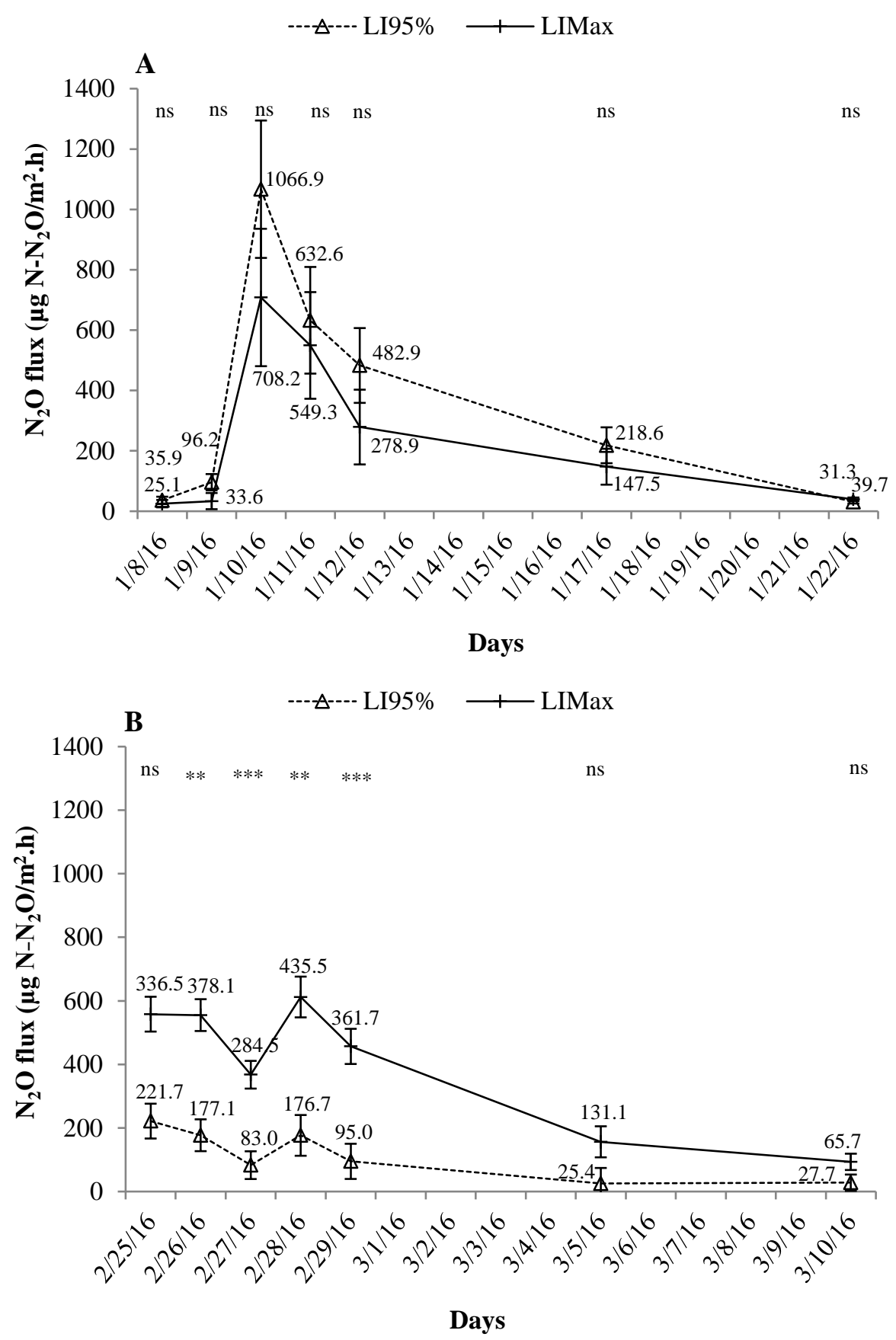

Figure 3. Nitrous oxide fluxes $\left(\mu \mathrm{g} \mathrm{N}-\mathrm{N}_{2} \mathrm{O} / \mathrm{m}^{2} . h\right)$ derived from soil established with elephant grass subjected to strategies of rotational stocking management ( $\mathrm{LI}_{95 \%}$ or $\mathrm{LI}_{\mathrm{Max}}$ ) during sampling periods $\mathrm{P}_{1}$ (A) and $\mathrm{P}_{2}(\mathrm{~B})$.

\subsubsection{Principal component analysis}

Principal component analysis generated six principal components, however, only the first two were explored because had eigenvalues greater than 1 (Kaiser criteria; Jolliffe, 2002) and accounted for $71.8 \%$ of the total variance in $\mathrm{N}_{2} \mathrm{O}$ fluxes (Table 4). The first principal component (PC1) explained $49 \%$ of the total variance and indicated high positive scores for $\mathrm{N}_{2} \mathrm{O}$ fluxes and WFPS, and high negative scores for soil and chamber temperatures. Analysis of variance on PC1 
scores showed a significant effect of sampling period $(P<0.01)$. The second principal component (PC2) accounted for $22.8 \%$ of the total variance and showed high positive score for soil $\mathrm{NH}_{4}{ }^{+}$and high negative score for soil $\mathrm{NO}_{3}{ }^{-}$contents. Analysis of variance on $\mathrm{PC} 2$ scores showed a significant effect of treatment $\times$ sampling period interaction $(P=0.0015)$.

Table 4. Coefficients of principal components based on the correlation matrix for $\mathrm{N}_{2} \mathrm{O}$ fluxes, soil $\mathrm{NH}_{4}{ }^{+}$and $\mathrm{NO}_{3}^{-}$, soil and chamber temperatures, and water-filled pore space from soil established with elephant grass subjected to strategies of rotational stocking management $\left(\mathrm{LI}_{95 \%}\right.$ or $\left.\mathrm{LI}_{\text {Max }}\right)$

\begin{tabular}{lcc}
\hline Variables & PC1 & PC2 \\
\hline $\mathrm{N}_{2} \mathrm{O}$ fluxes & 0.49 & -0.08 \\
$\mathrm{Soil} \mathrm{NH}_{4}{ }^{+}$ & 0.13 & 0.67 \\
$\mathrm{Soil}^{-}$ & 0.00 & -0.70 \\
Soil temperature & -0.48 & 0.19 \\
Chamber temperature & -0.49 & 0.07 \\
Water-filled pore space & 0.52 & 0.15 \\
\hline Eigenvalue & 2.94 & 1.37 \\
\% of variation explained & 49.0 & 22.8 \\
\hline ANOVA & \multicolumn{2}{c}{$\boldsymbol{P}$-value } \\
\hline Trt ${ }^{1}$ & 0.1149 & 0.6239 \\
Per & $<0.0001$ & 0.2950 \\
Trt $\times$ Per & 0.6934 & 0.0015 \\
\hline${ }^{2}$ Treatment effect & \multicolumn{2}{l}{}
\end{tabular}

\subsection{Discussion}

The grazing management strategies used in this study provided contrastant pre- and postgrazing SSH that affected grazing interval and ultimately the number of grazing cycles. For $\mathrm{LI}_{\mathrm{Max}}$, preand post-grazing SSH were 135 and $64 \mathrm{~cm}$, respectively, which resulted in an average grazing interval of 32 days and 3.5 grazing cycles during the experimental period (Congio et al., 2018). On the other hand, for $\mathrm{LI}_{95 \%}$, pre- and post-grazing $\mathrm{SSH}$ were 100 and $50 \mathrm{~cm}$, respectively, which resulted in an average grazing interval of 21 days and 5.6 grazing cycles (Congio et al., 2018). Considering adaptation and experimental periods (from January 2015 to April 2016) there were 9.3 grazing cycles for $\mathrm{LI}_{\mathrm{Max}}$ and 14.1 for $\mathrm{LI}_{95 \%}$, indicating greater frequency of defoliation on paddocks managed with the $\mathrm{LI}_{95 \%}$ target relative to those managed with the $\mathrm{LI}_{\mathrm{Max}}$ target. To keep the pre- and post-grazing targets, stocking rate was $33 \%$ greater for $\mathrm{LI}_{95 \%}$ than $\mathrm{LI}_{\mathrm{Max}}$ during the experimental period (9.3 vs. 7.0 cows/ha; Congio et al., 2018). These grazing conditions created different scenarios of intensification, solely by changing pre-grazing targets $\left(\mathrm{LI}_{95 \%}\right.$ or $\left.\mathrm{LI}_{\mathrm{Max}}\right)$. It is worthwhile to mention that the greater stocking rate obtained in $\mathrm{LI}_{95 \%}$ was supported by greater leaf accumulation and greater grazing 
efficiency rather than increased $\mathrm{N}$ fertilizer input, usually applied in intensive temperate pasture-based systems (Ramsbottom et al., 2015; Macdonald et al., 2017; Congio et al., 2018).

Emissions of $\mathrm{N}_{2} \mathrm{O}$ are a result of soil microbial nitrification and denitrification (de Klein and Eckard, 2008; Saggar et al., 2013). Both processes are mediated by soil properties such as mineral N (i.e. $\mathrm{NH}_{4}{ }^{+}$and $\mathrm{NO}_{3}{ }^{-}$) and organic carbon availabilities, moisture, $\mathrm{pH}$, temperature, and texture (de Klein et al., 2008; Luo et al., 2017). In grazed pastoral soils, the factors pointed out as key drivers of $\mathrm{N}_{2} \mathrm{O}$ fluxes are $\mathrm{N}$ inputs (i.e. urine patches and fertilizer) and WFPS (de Klein et al., 2008; Luo et al., 2017). Nitrous oxide fluxes and soil $\mathrm{NH}_{4}{ }^{+}$varied with LI pre-grazing target $\times$sampling period interaction, while a trend was observed for the effect of $\mathrm{NO}_{3}{ }^{-}$. On the other hand, the variables related to weather (i.e. WFPS, soil and chamber temperatures) varied only with sampling period. Most studies have indicated that high $\mathrm{N}_{2} \mathrm{O}$ emissions are usually associated with anaerobic soils with enough $\mathrm{NO}_{3}{ }^{-}$ supply suggesting that denitrification is the main process responsible for $\mathrm{N}_{2} \mathrm{O}$ emissions (de Klein and Eckard, 2008; de Klein et al., 2008). However, on excessively saturated soils with higher WFPS (i.e. optimal conditions for denitrification), as observed in $\mathrm{P}_{2}$, denitrification is complete and results in greater $\mathrm{N}_{2}: \mathrm{N}_{2} \mathrm{O}$ ratio (Bolan et al., 2004; de Klein et al., 2008). Although the accumulated rainfall was greater during $\mathrm{P}_{1}(199 \mathrm{~mm})$ than $\mathrm{P}_{2}(106 \mathrm{~mm})$, the WFPS was constantly greater throughout $\mathrm{P}_{2}$ than $\mathrm{P}_{1}$. These results are likely associated with better rainfall distribution during $\mathrm{P}_{2}$, where there were $80 \%$ of rainy days, while during $\mathrm{P}_{1}$ there were just $47 \%$ of rainy days.

The WFPS oscillation throughout $P_{1}$ followed the rainfall pattern. At day $3(1 / 10 / 16)$, a 39 $\mathrm{mm}$ rainfall increased WFPS from around 50 to more than $90 \%$ (Figure $2 \mathrm{~A}$ ), driving $\mathrm{N}_{2} \mathrm{O}$ fluxes up to $1000 \mu \mathrm{g} \mathrm{N}-\mathrm{N}_{2} \mathrm{O} / \mathrm{m}^{2}$.h (Figure 3A). Thenceforward, the WFPS was kept above $90 \%$ until day 10 $(1 / 17 / 16)$ and $\mathrm{N}_{2} \mathrm{O}$ fluxes decreased likely because of low oxygen availability that may have favored complete denitrification and $\mathrm{N}_{2}$ production (Bolan et al., 2004; de Klein et al., 2008). Throughout $\mathrm{P}_{2}$, the more uniform rainfall regime maintained WFPS above $90 \%$ with little oscillation until day 15 (3/10/16; Figure 2B), and $\mathrm{N}_{2} \mathrm{O}$ fluxes were kept moderate during the first half of $\mathrm{P}_{2}$, decreasing at the end of the period (Figure 3B). Studies have reported that the peak of $\mathrm{N}_{2} \mathrm{O}$ emissions occurs at WFPS values around $60-80 \%$, when simultaneous nitrification and denitrification were at maximum levels (Davidson, 1992; Rafique et al., 2011). Above this WFPS range, denitrification is the main source of $\mathrm{N}_{2} \mathrm{O}$ and under excessively anaerobic conditions, $\mathrm{N}_{2}: \mathrm{N}_{2} \mathrm{O}$ ratio remains greater (Bolan et al., 2004; de Klein et al., 2008; Rafique et al., 2011). The results of PCA pointed to an interaction among the driving factors regulating $\mathrm{N}_{2} \mathrm{O}$ fluxes from soil. The first principal component indicated that environmental factors (i.e. WFPS, soil and chamber temperatures) were determinants of $\mathrm{N}_{2} \mathrm{O}$ emissions and explained $49 \%$ of the whole dataset variability. Principal component two showed that factors related to LI pre-grazing targets (i.e. soil $\mathrm{NH}_{4}{ }^{+}$and $\mathrm{NO}_{3}{ }^{-}$) had the highest scores and accounted for $22.8 \%$ of total variance. Flechard et al. (2007) also reported that weather factors explained half of the total variability in their $\mathrm{N}_{2} \mathrm{O}$ flux dataset of ten sites for three years across Europe. Analysis of variance on PC1 and PC2 scores corroborated the results from the analysis of variance, where 
environmental factors showed significant effect for sampling period, as observed in PC1, and treatment related factors showed significant effect for the LI pre-grazing target $\times$ sampling period interaction, as observed in PC2.

Both soil $\mathrm{NH}_{4}{ }^{+}$and $\mathrm{NO}_{3}{ }^{-}$represented the concentration immediately after urea fertilization at day one, and therefore indicate $\mathrm{N}$ availability at the beginning of each sampling period. For both LI pre-grazing targets, a total of $215 \mathrm{~kg} \mathrm{~N} / \mathrm{ha}$ was applied throughout the experimental period. However, this amount was divided in 3.5 and 5.6 instalments for $\mathrm{LI}_{\mathrm{Max}}$ and $\mathrm{LI}_{95 \%}$, respectively. Therefore, the $\mathrm{N}$ inputs from urea fertilizer immediately before $\mathrm{N}_{2} \mathrm{O}$ sampling were greater for $\mathrm{LI}_{\mathrm{Max}}$ than $\mathrm{LI}_{95 \%}$ during $\mathrm{P}_{1}(75$ vs. $44 \mathrm{~kg} \mathrm{~N} / \mathrm{ha})$ and $\mathrm{P}_{2}(111$ vs. $57 \mathrm{~kg} \mathrm{~N} / \mathrm{ha})$. However, there was a significant treatment $\times$ sampling period interaction on soil $\mathrm{NH}_{4}{ }^{+}$concentration, most likely associated with urinary-N discharge. During $\mathrm{P}_{1}$, there was a greater urinary-N discharge for $\mathrm{LI}_{95}$ than $\mathrm{LI}_{\mathrm{Max}}(26.3$ vs. $20.9 \mathrm{~kg}$ of urinary-N/paddock) caused by higher stocking rate (10.0 vs. 8.3 cows/ha), which resulted in greater $\mathrm{N}_{2} \mathrm{O}$ fluxes for $\mathrm{LI}_{95 \%}$. Inversely, during $\mathrm{P}_{2}$, the soil $\mathrm{NH}_{4}{ }^{+}$and $\mathrm{N}_{2} \mathrm{O}$ fluxes were greater for $\mathrm{LI}_{\mathrm{Max}}$ than $\mathrm{LI}_{95 \%}$. During this period, the greater urinary-N discharge (46.8 vs. $44.8 \mathrm{~kg}$ of urinary-N/paddock) was likely associated with greater stocking period (1.88 vs. 1.46 days) for $\mathrm{LI}_{\text {Max }}$ relative to $\mathrm{LI}_{95 \%}$, since both treatments had similar stocking rate (9.5 vs. 9.9 cows/ha). These results are in agreement with most studies that have reported urine patches as the main source of $\mathrm{N}_{2} \mathrm{O}$ from grazed pasture soil mainly by providing highly localized concentrations of available $\mathrm{N}$, ranging from 200-2000 kg N/ha, associated with increased moisture and temperature conditions (Selbie et al., 2015; Luo et al., 2018).

Dairy farming systems based in temperate pastures are usually more intensive than tropical pasture-based dairy systems (Congio et al., 2018). Temperate forage crops were deeply studied and the understanding of their ecophysiology allowed for better use by farmers through adoption of adequate grazing management strategies, ensuring high milk productivity. The intensification of such systems is usually coupled with extra inputs of $\mathrm{N}$ fertilizer to boost forage growth or external supplementary feed, both aiming at increased stocking rate (Ramsbottom et al., 2015; Macdonald et al., 2017). In the tropics, dairy farming systems usually have low $\mathrm{N}$ inputs and adopt inadequate grazing management strategies resulting in low milk productivity. Therefore, the intensification of tropical pasture-based dairy systems is possible through adoption of adequate grazing strategies rather than extra $\mathrm{N}$ inputs or additional supplements, provided that minimum soil fertility to meet plant nutritional demand is ensured. The results indicated the opportunity to increase milk productivity in 52\% (170 and 112 $\mathrm{kg} / \mathrm{ha}$.day for $\mathrm{LI}_{95 \%}$ and $\mathrm{LI}_{\mathrm{Max}}$, respectively; Congio et al., 2018) only with adoption of strategic grazing management (i.e. $\mathrm{LI}_{95 \%}$ pre-grazing target).

Experimental approaches have shown that intensively managed pastures are greater sources of $\mathrm{N}_{2} \mathrm{O}$ than extensively managed pastures (Flechard et al., 2007; Rafique et al., 2011). Rafique et al. (2011) reported that frequently grazed sites that applied $400 \mathrm{~kg}$ of N/ha emitted two times more $\mathrm{N}_{2} \mathrm{O}$ compared to less frequently grazed sites that used around $300 \mathrm{~kg}$ of N/ha. However, in their study, intensively managed systems were generated through greater inputs of $\mathrm{N}$ fertilizer. In the present 
study, the more intensive grazing strategy was obtained through optimization of ecological processes rather than additional inputs of $\mathrm{N}$ fertilizer (Congio et al., 2018). Although urinary- $\mathrm{N}$ excretion has increased soil $\mathrm{NH}_{4}{ }^{+}$and ultimately $\mathrm{N}_{2} \mathrm{O}$ fluxes during $\mathrm{P}_{1}$ for $\mathrm{LI}_{95 \%}$, during $\mathrm{P}_{2}$ the urinary-N excretion and $\mathrm{N}_{2} \mathrm{O}$ fluxes were greater for $\mathrm{LI}_{\mathrm{Max}}$ counterbalancing the emissions for the entire experimental period ( $255 \mu \mathrm{g} \mathrm{N}-\mathrm{N}_{2} \mathrm{O} / \mathrm{m}^{2}$.h; $P=0.9975$ ). Converting hourly $\mathrm{N}_{2} \mathrm{O}$ fluxes to daily basis and relating to milk productivity, $\mathrm{LI}_{95 \%}$ was $35 \%$ more efficient than $\mathrm{LI}_{\mathrm{Max}}$ considering emissions for the entire period

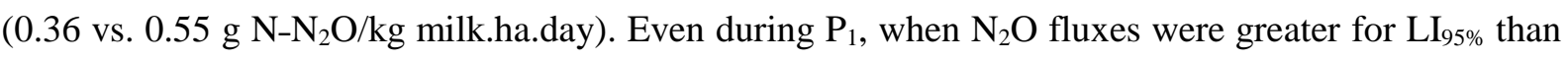
$\mathrm{LI}_{\mathrm{Max}}, \mathrm{LI}_{95 \%}$ emited less $\mathrm{N}-\mathrm{N}_{2} \mathrm{O} / \mathrm{kg}$ of milk.ha.day than $\mathrm{LI}_{\text {Max }}\left(0.61\right.$ vs. $0.68 \mathrm{~g} \mathrm{~N}-\mathrm{N}_{2} \mathrm{O} / \mathrm{kg}$ milk.ha.day). In addition, strategic grazing management decreased $34 \%$ urea-N applied per milk yield per hectare ( 0.57 vs. $0.86 \mathrm{~g}$ urea-N/kg milk.ha.day).

In a context where there is concern about the intensification of temperate pasture-based dairy systems through greater $\mathrm{N}$ fertilizer inputs, these findings highlight an opportunity to improve the efficiency of tropical pasture-based dairy systems through optimization of ecological processes. Strategic grazing allows for intensification that is not coupled with increases in inputs of external resources (i.e. fertilizer, external supplements) but rather with efficient use of existing resources (i.e. solar radiation, rainwater, pasture, fertilizer, supplement). Congio et al. (2018) have shown that strategic grazing management might reduce approximately $20 \%$ of enteric $\mathrm{CH}_{4}$ emission intensity and $\mathrm{CH}_{4}$ yield of dairy cows in tropical pasture-based systems. Carbon footprint in dairy farming systems is often dependent on emissions of enteric $\mathrm{CH}_{4}$ and $\mathrm{N}_{2} \mathrm{O}$ but also on carbon sequestration by forage crops with increase in soil organic carbon. Abdalla et al. (2018) revealed that the impact of grazing intensity on soil organic carbon is strongly climate dependent, and moist-warm regions present different responses than dry-warm, dry-cold and moist-cold climates. The authors highlighted that $\mathrm{C}_{4}$ grass species under high grazing intensities in moist-warm regions are more likely to increase soil organic carbon through enhanced plant turnover (i.e. root and litter) and excreta distribution than $\mathrm{C}_{4}$ under low grazing intensity. Then, the intensification of tropical pasture-based dairy farms through strategic grazing management uncoupled to $\mathrm{N}$ fertilizer increases could be a strategy for $\mathrm{GHG}$ mitigation. In addition, strategic grazing management is a noncost and readily available practice with easy adoption that enhances profitability of tropical pasture-based systems.

\subsection{Conclusions}

Nitrous oxide fluxes from grazed pastoral soils in moist-warm conditions is a very complex process regulated by environmental conditions and soil nitrogen availability. The central hypothesis that frequent defoliation provided by the $\mathrm{LI}_{95 \%}$ pre-grazing target would result in greater $\mathrm{N}_{2} \mathrm{O}$ fluxes from soil than less frequent defoliation (i.e. $\mathrm{LI}_{\mathrm{Max}}$ ) was not confirmed. However, these results associated with those of Congio et al. (2018) highlight that it is possible to intensify tropical pasture- 
based dairy systems through the adoption of adequate grazing strategies rather than extra $\mathrm{N}$ fertilizer inputs or additional supplements, as is usually pointed out for temperate grazing systems. This indicates the opportunity to significantly enhance milk productivity from tropical pasture-based systems using strategic grazing management ( $\mathrm{LI}_{95 \%}$; Congio et al., 2018) and decrease $35 \%$ the $\mathrm{N}-\mathrm{N}_{2} \mathrm{O}$ emitted per kg of milk. However, further farm-scale studies are necessary for a wide range of tropical grazed soils and dairy farming systems, associating not only GHG sources, but also the carbon sequestration by pasture soils, and milk productivity to achieve more accurate estimates of carbon balance and then to support mitigation plans by policy makers.

\section{References}

Abdalla, M., Hastings, A., Chadwick, D.R., Jones, D.L., Evans, C.D., Jones, M.B., Rees, R.M., Smith, P., 2018. Critical review of the impacts of grazing intensity on soil organic carbon storage and other soil quality indicators in extensively managed grasslands. Agric. Ecosyst. Environ. 253:6281. https://doi.org/10.1016/j.agee.2017.10.023.

Aguirre-Villegas, H.A., Passos-Fonseca, T.H., Reinemann, D.J., Larson, R.A., 2017. Grazing intensity affects the environmental impact of dairy systems. J. Dairy Sci. 100: 6804-6821. https://doi.org/10.3168/jds.201612325.

Alves, B.J.R., Smith, K.A., Flores, R.A., Cardoso, A.S., Oliveira, W.R.D., Jantalia, C.P., Urquiaga, S., Boddey, R.M., 2012. Selection of the most suitable sampling time for static chambers for the estimation of daily mean $\mathrm{N}_{2} \mathrm{O}$ flux from soils. Soil Biol. Biochem. 46:129-135. https://doi.org/10.1016/j.soilbio.2011.11.022.

Auerswald, I.C., Mayer, F. Schnyder, H. 2010. Coupling of spatial and temporal pattern of cattle excreta patches on a low intensity pasture. Nutr. Cycl. Agroecosyst. 88:275-288. https://doi.org/10.1007/s10705-009-9321-4.

Bardgett, R.D, Jones, A.C., Jones, D.L., Kemmitt, S.J., Cook, R., Hobbs, P.J., 2001. Soil microbial community patterns related to the history and intensity of grazing in sub-montane ecosystems. Soil Biol. Biochem. 33:1653-1664. https://doi.org/10.1016/S0038-0717(01)00086-4.

Bardgett, R.D., Hobbs, P.J., Frostegard, A., 1996. Changes in soil fungal:bacterial biomass ratios following reductions in the intensity of management of an upland grassland. Biol. Fertil. Soils. 22:261-264. https://doi.org/10.1007/BF00382522.

Bardgett, R.D., Wardle, D.A., 2003. Herbivore-mediated linkages between aboveground and belowground communities. Ecology. 84:2258-2268. https://doi.org/10.1890/02-0274.

Barneze, A.S., Mazzetto, A.M., Zani, C.F., Misselbrook, T., Cerri, C.C., 2014. Nitrous oxide emissions from soil due to urine deposition by grazing cattle in Brazil. Atmos. Environ. 92:394397. https://doi.org/10.1016/j.atmosenv.2014.04.046. 
Barneze, A.S., Minet, E.P., Cerri, C.C., Misselbrook, T., 2015. The effect of nitrification inhibitors on nitrous oxide emissions from cattle urine deposition to grassland under summer conditions in the UK. Chemosphere. 119:122-129. https://doi.org/10.1016/j.chemosphere.2014.06.002.

Bolan, N.S., Saggar, S., Luo, J., Bhandral, R., Singh, J., 2004. Gaseous emissions of nitrogen from grazed pastures: processes, measurements and modelling, environmental implications, and mitigation. Adv. Agron. 84:37-120. https://doi.org/10.1016/S0065-2113(04)84002-1.

Carnevalli, R.A., Da Silva, S.C., Bueno, A.A.O., Uebele, M.C., Bueno, F.O., Hodgson, J., Silva, G.N., Morais, J.P.G., 2006. Herbage production and grazing losses in Panicum maximum cv. Mombaça under four grazing management. Trop. Grassl.-Forrajes Trop. 40:165-176. http://tropicalgrasslands.info/public/journals/4/Historic/Tropical\%20Grasslands\%20Journal\%20ar chive/PDFs/Vol 40 2006/Vol $40 \quad 03 \quad 2006$ pp165 176.pdf.

Carvalho, P.C.F., 2013. Harry Stobbs memorial lecture: can grazing behavior support innovations in grassland management? Trop. Grassl.-Forrajes Trop. 1:137-155. https://doi.org/10.17138/TGFT(1)137-155.

CEPAGRI, 2012. Centro de pesquisas meteorológicas e climáticas aplicadas à agricultura. [Center of Applied Climatic and Meteorological Research in Agriculture]. UNICAMP, Campinas. http://www.cpa.unicamp.br/outras-informacoes/clima_muni_436.html.

Chiavegato, M.B., Powers, W., Carmichael, D., Rowntree, J., 2015. Pasture-derived greenhouse gas emissions in cow-calf production systems. J. Anim. Sci. 93:1350-1364. https://doi.org/10.2527/jas2014-8134.

Clough, T.J., Lanigan, G.J., de Klein, C.A.M., Samad, M.S., Morales, S.E., Rex, D., Bakken, L.R., Johns, C., Condron, L.M., Grant, J., Richards, K.G., 2017. Influence of soil moisture on codenitrification fluxes from a urea-affected pasture soil. Sci. Rep. 7:2185. https://doi.org/10.1038/s41598-017-02278-y.

Congio, G.F.S., Batalha, C.D.A., Chiavegato, M.B., Berndt, A., Oliveira, P.P.A., Frighetto, R.T.S., Maxwell, T.M.R., Gregorini, P., Da Silva, S.C., 2018. Strategic grazing management towards sustainable intensification at tropical pasture-based dairy systems. Sci. Total Environ. 636:872880. https://doi.org/10.1016/j.scitotenv.2018.04.301.

Da Silva, S.C., Bueno, A.A.O., Carnevalli, R.A., Uebele, M.C., Bueno, F.O., Hodgson, J.,Matthew, C., Arnold, J.C., Morais, J.P.G., 2009. Sward structural characteristics and herbage accumulation of Panicum maximum cv. Mombaça subject to rotational stocking managements. Sci. Agric. 66:819. https://doi.org/10.1590/S010390162009000100002.

Da Silva, S.C., Chiavegato, M.B., Pena, K.S., Silveira, M.C.T., Barbero, L.M., Junior, S.J.S., Rodrigues, C.S., Limão, V.A., Pereira, L.E.T., 2017. Tillering dynamics of Mulato grass subjected to strategies of rotational grazing management. J. Agric. Sci. 155:1082-1092. https://doi.org/10.1017/S0021859617000223. 
Da Silva, S.C., Sbrissia, A.F., Pereira, L.E.T., 2015. Ecophysiology of C4 forage grassesunderstanding plant growth for optimising their use and management. Agriculture 5:598-625. https://doi.org/10.3390/agriculture5030598.

Davidson, E.A., 1992. Sources of nitric oxide and nitrous oxide following wetting of dry soil. Soil Sci. Soc. Am. J. 56:95-102. https://doi.org/10.2136/sssaj1992.03615995005600010015x.

de Klein, C.A.M, Eckard, R.J., 2008. Targeted technologies for nitrous oxide abatement from animal agriculture. Aust. J. Exp. Agric. 48:14-20. https://doi.org/10.1071/EA07217.

de Klein, C.A.M, Luo, J., Woodward, K.B., Styles, T., Wise, B., Lindsey, S., Cox, N., 2014. The effect of nitrogen concentration in synthetic cattle urine on nitrous oxide emissions. Agric. Ecosyst. Environ. 188:85-92. https://doi.org/10.1016/j.agee.2014.02.020.

de Klein, C.A.M., Harvey, M., 2015. Nitrous Oxide Chamber Methodology Guidelines. Ministry for Primary Industries, Pastoral House, 25 The Terrace, PO Box 2526, Wellington 6140, New Zealand, $\quad$ p. $\quad 146 . \quad$ https://globalresearchalliance.org/wpcontent/uploads/2015/11/Chamber_Methodology_Guidelines_Final-V1.1-2015.pdf.

de Klein, C.A.M., Pinares-Patino, C., Waghorn, G.C., 2008. Greenhouse gas emissions. In: McDowell, R.W. (Ed.), Environmental Impacts of Pasture Based Farming. CAB International, Wallingford, Oxfordshire, UK, 1-32. https://doi.org/10.1079/9781845934118.0001.

Di, H.J., Cameron, K.C., Podolyan, A., Edwards, G.R., de Klein, C.A.M., Dynes, R., Woods, R., 2016. The potential of using alternative pastures, forage crops and gibberellic acid to mitigate nitrous oxide emissions. J. Soils Sediments. 16:2252-2262. https://doi.org/10.1007/s11368-016-1442-1.

Flechard, C.R., Ambus, P., Skiba, U., Rees, R.M., Hensen, A., van Amstel, A., Pol-van Dasselaar, A.V., Soussana, J.F., Jones, M., Clifton-Brown, J., Raschi, A., Horvath, L., Neftel, A., Jocher, M., Ammann, C., Leifeld, J., Fuhrer, J., Calanca, P., Thalman, E., Pilegaard, K., Di Marco, C., Campbell, C., Nemitz, E., Hargreaves, K.J., Levy, P.E., Ball, B.C., Jones, S.K., van de Bulk, W.C.M., Groot, T., Blom, M., Domingues, R., Kasper, G., Allard, V., Ceschia, E., Cellier, P., Laville, P., Henault, C., Bizouard, F., Abdalla, M., Williams, M., Baronti, S., Berretti, F., Grosz, B., 2007. Effects of climate and management intensity on nitrous oxide emissions in grassland systems across Europe. Agric. Ecosyst. Environ. 121:135-152. https://doi.org/10.1016/j.agee.2006.12.024.

Fonseca, L., Mezzalira, J.C., Bremm, C., Filho, R.S.A., Gonda, H.L., Carvalho, P.C.F., 2012. Management targets formaximising the short-term herbage intake rate of cattle grazing in Sorghum bicolor. Livest. Sci. 145:205-211. https://doi.org/10.1016/j.livsci.2012.02.003.

Gardiner, C.A., Clough, T.J., Cameron, K.C., Di, H.J., Edwards, G.R., de Klein, C.A.M., 2016. Potential for forage diet manipulation in New Zealand pasture ecosystems to mitigate ruminant urine derived N2O emissions: a review. N. Z. J. Agric. Res. 59(3):301-317. https://doi.org/10.1080/00288233.2016.1190386. 
Gardiner, C.A., Clough, T.J., Cameron, K.C., Di, H.J., Edwards, G.R., de Klein, C.A.M., 2016. Potential inhibition of urine patch nitrous oxide emissions by Plantago lanceolata and its $\begin{array}{llllll}\text { metabolite aucubin. } & \text { N. }\end{array}$ https://doi.org/10.1080/00288233.2017.1411953.

Gimenes, F.M.A., Da Silva, S.C., Fialho, C.A., Gomes, M.B., Berndt, A., Gerdes, L., Colozza, M.T., 2011. Ganho de peso e produtividade animal em capim-marandu sob pastejo rotativo e adubação nitrogenada. Pesq. Agropec. Bras. 46:751-759. https://doi.org/10.1590/S0100204X2011000700011.

Gregorini, P., Beukes, P.C., Dalley, D., Romera, A.J., 2016. Screening for diets that reduce urinary nitrogen excretion and methane emissions while maintaining or increasing production by dairy cows. Sci. Total Environ. 551-552:32-41. https://doi.org/10.1016/j.scitotenv.2016.01.203.

Guerci, M., Knudsen, M.T., Bava, L., Zucali, M., Schonbach, P., Kristensen, T., 2013. Parameters affecting the environmental impact of a range of dairy farming systems in Denmark, Germany and Italy. J. Clean. Prod. 54:133-141. https://doi.org/10.1016/j.jclepro.2013.04.035.

IPCC, 2014. Summary for policymakers. Pages 6-10 in Climate Change 2014: Mitigation of Climate Change. Contribution of Working Group III to the Fifth Assessment Report of the Intergovernmental Panel on Climate Change (IPCC). O. Edenhofer, R. Pichs-Madruga, Y. Sokona, E. Farahani, S. Kadner, K. Seyboth, A. Adler, I. Baum, S. Brunner, P. Eickemeier, B. Kriemann, J. Savolainen, S. Schlomer, C. von Stechow, T. Zwickel, and J.C. Minx, ed. Cambridge University Press, Cambridge, UK. https://www.ipcc.ch/pdf/assessment-report/ar5/wg3/WGIIIAR5_SPM_TS_Volume.pdf.

Jarvis, S.C., Lovell, R.D., Panayides, R., 1995. Patterns of methane emissions from excreta of grazing cattle. Soil Biol. Biochem. 27:1581-1588. https://doi.org/10.1016/0038-0717(95)00092-S.

Jolliffe, I.T., 2002. Principal component analysis. 2nd edn. New York: Springer-Verlag. pp. 518. http://cda.psych.uiuc.edu/statistical_learning_course/Jolliffe\%20I.\%20Principal\%20Component\% 20Analysis\%20(2ed.,\%20Springer,\%202002)(518s)_MVsa_.pdf

Lessa, A.C.R., Madari, B.E., Paredes, D.S., Boddey, R.M., Urquiaga, S., Jantalia, C.P., Alves, B.J.R., 2014. Bovine urine and dung deposited on Brazilian savannah pastures contribute differently to direct and indirect soil nitrous oxide emissions. Agr. Ecosyst. Environ. 190:104-111. https://doi.org/10.1016/j.agee.2014.01.010.

Levine, U.Y., Teal, T.K., Robertson, G.P., Schmidt, T.M., 2011. Agriculture's impact on microbial diversity and associated fluxes of carbon dioxide and methane. ISME J. 5:1683-1691. https://doi.org/10.1038/ismej.2011.40. 
Luo, J., Wyatt, J., van der Weerden, T., Thomas, S., de Klein, C., Li, Y., Rollo, M., Lindsey, S., Ledgard, S., Li, J., Ding, W., Qin, S., Zhang, N., Bolan, N., Kirkham, M.B., Bai, Z., Ma, L., Zhang, X., Wang, H., Liu, H., Rys, G., 2017. Potential hotspot areas of nitrous oxide emissions from grazed pastoral dairy farm systems. Adv. Agron. 145:205-268. https://doi.org/10.1016/bs.agron.2017.05.006.

Luo, J., Balvert, S.F., Wise, B., Welten, B., Ledgard, S.F., de Klein, C.A.M., Lindsey, S., Judge, A., 2018. Using alternative forage species to reduce emissions of the greenhouse gas nitrous oxide from cattle urine deposited onto soil. Sci. Total Environ. 610-611:1271-1280. https://doi.org/10.1016/j.scitotenv.2017.08.186.

Macdonald, K.A., Penno, J.W., Lancaster, J.A.S., Bryant, A.M., Kidd, J.M., Roche, J.R., 2017. Production and economic responses to intensification of pasture-based dairy production systems. J. Dairy Sci. 100:6602-6619. https://doi.org/10.3168/jds.2016-12497.

Mazzetto, A.M., Barneze, A.S., Feigl, B.J., van Groenigen, J.W., Oenema, O., Cerri, C.C., 2014. Temperature and moisture affect methane and nitrous oxide emission from bovine manure patches in tropical conditions. Soil Biol Biochem 76:242-248. https://doi.org/10.1016/j.soilbio.2014.05.026.

Mazzetto, A.M., Barneze, A.S., Feigle, B.J., van Groenigen, J.W., Oenema, O., de Klein, C.A.M., Cerri, C.C., 2015. Use of the nitrification inhibitor dicyandiamide (DCD) does not mitigate N2O emission from bovine urine patches under Oxisol in Northwest Brazil. Nutr. Cycl. Agroecosyst. 101:83-92. https://doi.org/10.1007/s10705-014-9663-4.

Myhre, G., Shindell, D., Bréon, F.-M., Collins, W., Fuglestvedt, J., Huang, J., Koch, D., Lamarque, J.F., Lee, D., Mendoza, B., Nakajima, T., Robock, A., Stephens, G., Takemura, T., Zhang, H., 2013. Anthropogenic and natural radiative forcing. In: Stocker, T.F., Qin, D., Plattner, G.-K., Tignor, M., Allen, S.K., Boschung, J., Nauels, A., Xia, Y., Bex, V., Midgley, P.M. (Eds.), Climate Change 2013: the Physical Science Basis. Contribution of Working Group I to the Fifth Assessment Report of the Intergovernmental Panel on Climate Change. Cambridge University Press, Cambridge, United Kingdom and New York, NY, USA, pp. 659-740. https://doi.org/10.1017/CBO9781107415324.018.

O’Brien, D., Shalloo, L., Patton, J., Buckley, F., Grainger, C., Wallace, M., 2012. A life cycle assessment of seasonal grass-based and confinement dairy farms. Agr. Syst. 107:33-46. https://doi.org/10.1016/j.agsy.2011.11.004.

Pereira, L.E.T., Paiva, A.J., Geremia, E.V., Da Silva, S.C., 2014. Components of herbage accumulation in elephant grass cvar Napier subjected to strategies of intermittent stocking management. J. Agric. Sci. 152:954-966. https://doi.org/10.1017/S0021859613000695.

Pereira, L.E.T., Paiva, A.J., Geremia, E.V., Da Silva, S.C., 2015. Regrowth patterns of elephant grass (Pennisetum purpureum Schum.) subjected to strategies of intermittent stocking management. Grass Forage Sci. 70:195-204. https://doi.org/10.1111/gfs.12103. 
Rafique, R., Hennessy, D., Kiely, G., 2011. Nitrous oxide emission from grazed grassland under different management systems. Ecosystems. 14:563-582. https://doi.org/10.1007/s10021-0119434-x.

Ramsbottom, G., Horan, B., Berry, D.P., Roche, J.R., 2015. Factors associated with the financial performance of spring-calving, pasture-based dairy farms. J. Dairy Sci. 98: 3526-3540. https://doi.org/10.3168/jds.20148516.

Ravishankara, A.R, Daniel, J.S, Portmann, R.W., 2009. Nitrous oxide $\left(\mathrm{N}_{2} \mathrm{O}\right)$ : the dominant ozonedepleting substance emitted in the 21st century. Science. 326:123-5. https://doi.org/10.1126/science.1176985.

Rex, D., Clough, T.J., Richards, K.G., de Klein, C.A.M., Morales, S.E., Samad, M.S., Grant, J., Lanigan, G.J., 2018. Fungal and bacterial contributions to codenitrification emissions of N2O and N2 following urea deposition to soil. Nutr. Cycl. Agroecosyst. 110(1):135-149. https://doi.org/10.1007/s10705-017-9901-7.

Saggar, S., Giltrap, D.L., Li, C., Tate, K.R., 2007. Modelling nitrous oxide emissions from grazed grasslands in New Zealand. Agric. Ecosyst. Environ. 119:205-16. https://doi.org/10.1016/j.agee.2006.07.010.

Saggar, S., Jha, N., Deslippe, J., Bolan, N.S., Luo, J., Giltrap, D.L., Kim, D.G., Zaman, M., Tillman, R.W., 2013. Denitrification and N2O:N2 production in temperate grasslands: process, measurements, modelling and mitigating negative impacts. Sci. Total Environ. 465:173-195. https://doi.org/10.1016/j.scitotenv.2012.11.050.

Samad, M.D.S., Biswas, A., Bakken, L.R., Clough, T.J., de Klein, C.A.M., Richards, K.G., Lanigan, G.J., Morales, S.E., 2016. Phylogenetic and functional potential links pH and N2O emissions in pasture soils. Sci. Rep. 6:35990. https://doi.org/10.1038/srep35990.

Schmalz, H.J., Taylor, R.V., Johnson, T.N., Kennedy, P.L., DeBano, S.J., Newingham, B.A., McDaniel, P.A., 2013. Soil Morphologic properties and cattle stocking rate affect dynamic soil properties. Rangeland Ecol. Manag. 66(4):445-453. https://doi.org/10.2111/REM-D-12-00040.1.

Selbie, D.R., Buckthought, L.E., Shepherd, M.A., 2015. The challenge of the urine patch for managing nitrogen in grazed pasture systems. Adv. Agron. 129:229-292. http://doi.org/10.1016/bs.agron.2014.09.004.

Silva, A.P., Imhoff, S., Corsi, M., 2003. Evaluation of soil compaction in irrigated short-duration grazing system. Soil Tillage Res. 70:83-90. https://doi.org/10.1016/S0167-1987(02)00122-8.

Silveira, M.C.T., Da Silva, S.C., Souza Jr., S.J., Barbero, L.M., Rodrigues, C.S., Limão, V.A., Pena, K.S., Nascimento Jr., D., 2013. Herbage accumulation and grazing losses on Mulato grass subjected to strategies of rotational stocking management. Sci. Agric. 70:242-249. https://doi.org/10.1590/S0103-90162013000400004. 
Smith, P., Goulding, K.W., Smith, K.A., Powlson, D.S., Smith, J.U., Falloon, P., Coleman, K., 2001. Enhancing the carbon sink in European agricultural soils: Including trace gas fluxes in estimates of carbon mitigation potential. Nutr. Cycl. Agroecosyst. 60:237-252. https://doi.org/10.1023/A:1012617517839.

van der Weerden, T.J., Styles, T.M., Rutherford, A.J., de Klein, C.A.M., Dynes, R., 2017. Nitrous oxide emissions from cattle urine deposited onto soil supporting a winter forage kale crop. N. Z. J. Agric. Res. 60:119-130. https://doi.org/10.1080/00288233.2016.1273838.

Venterea, R., Clough, T.J., Coulter, J.A., Breuillin-Sessoms, F., Wang, P., Sadowsky, M.J., 2015. Ammonium sorption and ammonia inhibition of nitrite-oxidizing bacteria explain contrasting soil N2O production. Sci. Rep. 5:12153. https://doi.org/10.1038/srep12153.

Venterea, R.T., 2010. Simplified method for quantifying theoretical underestimation of chamber-based trace gas fluxes. J. Environ. Qual. 39:126-135. https://doi.org/10.2134/jeq2009.0231.

Vibart, R.E., Tavendale, M., Otter, D., Schwendel, B.H., Lowe, K., Gregorini, P., Pacheco, D., 2017. Milk production and composition, nitrogen utilization, and grazing behavior of late-lactation dairy cows as affected by time of allocation of a fresh strip of pasture. J. Dairy Sci. 100:1-14. http://dx.doi.org/10.3168/jds.2016-12413.

Voltolini, T.V., Santos, F.A.P., Martinez, J.C., Imaizumi, H., Clarindo, R.L., Penati, M.A., 2010. Produção e composição do leite de vacas mantidas em pastagens de capim-elefante submetidas a duas frequências de pastejo. Rev. Bras. Zootecn. 39 (1):121-127. https://doi.org/10.1590/S1516$\underline{35982010000100016 .}$

Warren, S.D., Thurow, T.L., Blackburn, W.H., Garza, N.E., 1986. The influence of livestock trampling under intensive rotation grazing on soil hydrologic characteristics. J. Range Manage. 39:491-495. https://doi.org/10.2307/3898755.

White, L.M., 1973. Carbohydrate reserves of grasses: a review. J. Range Manage. 26: 13-18. https://doi.org/10.2307/3896873.

Wrage, N., Velthof, G.L., van Beusichem, M.L., Oenema, O., 2001. Role of nitrifier denitrification in the production of nitrous oxide. Soil Biol. Biochem. 33:1723-1732. https://doi.org/10.1016/S0038-0717(01)00096-7. 


\title{
5. EFFECTS OF TIMING OF PADDOCK ALLOCATION ON MILK YIELD AND ENTERIC METHANE EMISSIONS FROM DAIRY COWS
}

\begin{abstract}
Dairy products are major components of the human diet. Pasture-based systems are important milk suppliers to dairy industry in temperate and tropical climates and thereby will play relevant role to support the growing demand. However, this additional milk supply must be obtained through higher yields resulting from intensification of existing farming systems using environmentally friendly and economically profitable strategies towards sustainable intensification. The objective of this study was to investigate the influence of timing of paddock allocation (AM or PM) on the nutritive value of rotationally managed elephant grass (Pennisetum purpureum Schum. cv. Cameroon), and the dry matter intake (DMI), milk yield, milk composition, and enteric methane $\left(\mathrm{CH}_{4}\right)$ emissions of Holstein $\times$ Jersey dairy cows. The hypothesis was that new paddock allocation to dairy cows in the afternoon, when herbage has greater nutritive value, increases nutrient intake and milk yield, and reduces enteric $\mathrm{CH}_{4}$ emissions per $\mathrm{kg}$ of milk, relative to paddock allocation in the morning. Herbage sampled in the afternoon had greater dry matter, soluble carbohydrates, starch, and non-fibrous carbohydrate/protein ratio, and lesser neutral-detergent fiber and acid-detergent fiber concentrations. There was no treatment effect on milk yield. However, protein and casein yields tended to be greater for PM than AM. Milk urea nitrogen was greater for cows grazing paddocks allocated during the morning relative to those allocated in the afternoon. The timing of paddock allocation did not affect DMI, daily enteric $\mathrm{CH}_{4}$ emission, and enteric $\mathrm{CH}_{4}$ per $\mathrm{kg}$ of milk. The results ratify the general understanding of diurnal variation in herbage chemical composition. However, the increase in nutritive value of the afternoon relative to the morning herbage was not enough to increase DMI and milk yield, or to decrease $\mathrm{CH}_{4}$ emission intensity by the dairy cows as hypothesized. The findings also indicate that new paddock allocation during the afternoon can be a simple and useful grazing strategy that results in greater $\mathrm{N}$ partitioning to protein yield, and lower excretion of urea $\mathrm{N}$ in milk.
\end{abstract}

Keywords: Timing of paddock allocation; Enteric methane emissions; Herbage quality; Diurnal variation; Non-fibrous carbohydrate; Elephant grass

\subsection{Introduction}

Dairy products are major components of the human diet (Aguirre-Villegas et al., 2017). Pasture-based systems are important milk suppliers to dairy industry in temperate (Chapman, 2016; Macdonald et al., 2017) and tropical climates (Santos et al., 2014; de Souza et al., 2017) and thereby will play relevant role to support the growing demand (Godfray et al., 2010; Conforti, 2011; Alexandratos and Bruinsma, 2012). However, this additional milk supply must be obtained through higher yields resulting from intensification of existing farming systems using environmentally friendly (Tilman et al., 2002) and economically profitable (Foote et al., 2015; Gregorini et al., 2017) strategies towards sustainable intensification (Godfray et al., 2010; Congio et al., 2018).

Several studies have reported diurnal variations in herbage chemical composition (Lechtenberg et al., 1971; Orr et al., 1997; Ciavarella et al., 2000; Griggs et al., 2005; Gregorini et al., 2006; Shewmaker et al., 2006; Gregorini et al., 2008; Morin et al., 2011). Such variations were 
attributed to the balance among processes of plant photosynthesis, plant respiration, and plant transpiration that results in greater non-fibrous carbohydrate (NFC) and dry matter (DM) accumulation from dawn to dusk (Curtis, 1944; Lechtenberg et al., 1971). The increase in NFC and DM concentrations mainly occur in the upper layers of the canopy (Delagarde et al., 2000), often diluting fiber and nitrogen (N) concentrations (Gregorini, 2012; Vibart et al., 2017), and enhancing herbage biomechanical properties (Gregorini et al., 2009) and digestibility (Burns et al., 2007; Pelletier et al., 2010; De Oliveira et al., 2018). Therefore, temporal patterns of herbage intake, ingestive and digestive behavior of grazing ruminants can be altered by timing of new strip or paddock allocation to grazing animals in rotationally managed pastures (Gibb et al., 1998; Orr et al., 2001; Gregorini et al., 2006; Gregorini et al., 2008; Abrahamse et al., 2009; Gregorini, 2012; Pulido et al., 2015; Vibart et al., 2017). Although these studies have not reported that such modifications in the herbage chemical composition can increase daily dry matter intake (DMI), Gregorini (2012) suggested that ruminants moved to a new fresh paddock in the afternoon might increase their nutrient intake because of longer and more intensive grazing during dusk, when herbage nutritive value is at its peak.

Enteric $\mathrm{CH}_{4}$ is the predominant source of greenhouse gases (GHG) emissions in dairy systems (Crosson et al., 2011; Aguirre-Villegas et al., 2017) and represent more than $80 \%$ of total GHG emissions in pasture-based farming systems (Guerci et al., 2013). According to Janssen (2010), the nature and amount of feed (e.g. herbage chemical composition and DMI, respectively) are key determinants of enteric $\mathrm{CH}_{4}$ emissions from ruminants. Modeling studies have shown possible reductions on enteric $\mathrm{CH}_{4}$ emissions intensity ( $\mathrm{g} / \mathrm{kg}$ of milk) by dairy cows when herbage NFC increases at the expense of fiber concentrations (Ellis et al., 2012), and Gregorini (2012) suggested the need of field research to assess this hypothesis.

The objective of this study was to investigate the influence of timing of paddock allocation (AM or PM) on the nutritive value of rotationally managed elephant grass (Pennisetum purpureum Schum. cv. Cameroon), and the DMI, milk yield, milk composition, and enteric $\mathrm{CH}_{4}$ emissions of Holstein $\times$ Jersey dairy cows. The hypothesis was that new paddock allocation to dairy cows in the afternoon, when herbage has greater nutritive value, increases nutrient intake and milk yield, and reduces enteric $\mathrm{CH}_{4}$ emissions per $\mathrm{kg}$ of milk, relative to paddock allocation in the morning.

\subsection{Material and Methods}

All procedures for this study were approved by the Animal (15.5.1246.11.2) and Environment Ethics Committees (17.5.999.11.9) at the University of São Paulo, College of Agriculture "Luiz de Queiroz" (USP/ESALQ). 


\subsubsection{Study site}

The experiment was conducted from January to March 2017 in Piracicaba, SP, Brazil $\left(22^{\circ} 42^{\prime} \mathrm{S}, 47^{\circ} 38^{\prime} \mathrm{W}\right.$ and 546 a.s.1.) on a rainfed, non-irrigated elephant grass pasture (Pennisetum purpureum Schum. cv. Cameroon) established in 1972 in a high fertility Eutroferric Red Nitossol. The climate is sub-tropical with dry winters and $1328 \mathrm{~mm}$ average annual rainfall (CEPAGRI, 2012). The mean temperature and accumulated rainfall during the experiment were $24.5{ }^{\circ} \mathrm{C}$ and $407 \mathrm{~mm}$ respectively.

\subsubsection{Treatments and experimental design}

The 3.3 ha experimental area was divided up into two farmlets of 24 paddocks each $\left(688 \mathrm{~m}^{2}\right.$ on average), and managed using a common rotational grazing strategy with one day of occupation. Pre- and post-grazing sward surface heights (SSH) were 100 and $55 \mathrm{~cm}$, respectively, which were found to optimize grazing efficiency and feeding value of elephant grass cv. Cameroon (Congio et al., 2018). Paddocks were subjected to a period of 11 months prior to the beginning of the experiment aiming to adapt sward structure to the grazing strategy used.

The two treatments corresponded to timings of herd allocation to a new paddock, either after morning milking at 6:00 am (AM) or after afternoon milking at 4:00 pm (PM). The experimental design was a randomized complete block, with eight replications, with slope and chemical soil characteristics used as blocking criteria. Each paddock received $56 \mathrm{~kg} \mathrm{~N} / \mathrm{ha}$ (as urea) during the experiment splitted in 2 instalments. Fertilizer application was made soon after grazing. The experimental period was divided in two sampling periods of 4 weeks each $\left(\mathrm{P}_{1}\right.$ and $\left.\mathrm{P}_{2}\right)$ and measurements were made during the last 7 days of each sampling period.

\subsubsection{Plant measurements}

The SSH was measured from ground level to top leaf horizon by 40 systematic readings, using a stick graduated in centimeters (Pereira et al., 2015a; Congio et al, 2018). Pre-grazing herbage mass was quantified in each grazing cycle on three rectangular samples collected randomly $\left(0.94 \mathrm{~m}^{2}\right.$ each). Herbage was clipped above the target post-grazing SSH, weighed fresh, and sub-sampled to determine plant-part components by hand separation into leaf (leaf blades), stem (stems + leaf sheaths) and dead material (Pereira et al., 2015b; Congio et al, 2018). Herbage allowance was calculated by the relationship between pre-grazing herbage mass (above post-grazing SSH) and number of cows per day (Pérez-Prieto and Delagarde, 2013; Congio et al, 2018).

Herbage samples to determine chemical composition were taken daily during the last 7 days of each sampling period $\left(\mathrm{P}_{1}\right.$ and $\left.\mathrm{P}_{2}\right)$ immediately before herd allocation to paddocks (6 am and $\left.4 \mathrm{pm}\right)$. 
Herbage was clipped above target post-grazing SSH at ten randomized sampling sites per paddock, homogenized, sub-sampled, freeze-dried, and ground through a 1-mm screen (Wiley Mill, Thomas Scientific, Philadelphia, PA). Dry matter (DM) and ash concentrations were determined at $105^{\circ} \mathrm{C}$ for $24 \mathrm{~h}$ and $600{ }^{\circ} \mathrm{C}$ for $4 \mathrm{~h}$, respectively (AOAC International, 2005). Neutral-detergent fiber (NDF), acid-detergent fiber (ADF) and lignin concentrations were determined sequentially (Van Soest et al., 1991). Ether extract (EE) concentration was determined according to AOAC International (2005). Total N concentration was determined by the Dumas combustion method using N analyzer (Leco FP2000 N Analyzer; Leco Instruments Inc., St. Joseph, MI, USA), and crude protein (CP) concentration calculated as $\mathrm{N} \times 6.25$. Neutral-detergent insoluble crude protein (NDICP), acid detergent-insoluble crude protein (ADICP), and soluble N concentrations were analyzed according to Licitra et al. (1996), and $\mathrm{N}$ fractions were determined by methodology adapted from Sniffen et al. (1992). Soluble carbohydrates in $80 \%$ ethanol-solution (SC) and starch concentrations were determined according to Hall (2003).

\subsubsection{Herd and feeding}

Twenty Holstein $\times$ Jersey dairy cows averaging $461 \pm 72 \mathrm{~kg}$ body weight (BW) and $2.83 \pm$ 0.23 body corporal score (BCS) were used. Four weeks prior to the experiment, all cows were managed in a single herd grazing elephant grass cv. Cameroon and receiving $6 \mathrm{~kg}$ (fresh basis) of commercial concentrate daily. Cows were then stratified, grouped in pairs and allocated to 10 blocks according to pre-experimental milk yield $(18.6 \pm 4.6 \mathrm{~kg} / \mathrm{d})$ and days in milk (102 $\pm 82 \mathrm{DIM})$. Within pairs, cows were randomly assigned to treatments (AM and PM).

Concentrate meals were fed individually twice daily (4:30 am and 2:30 pm) before milking ( $5 \mathrm{am}$ and $3 \mathrm{pm}$ ) at a rate of $1 \mathrm{~kg}$ of concentrate $/ 3 \mathrm{~kg}$ of milk (considering the average of each block). The rate was established based on milk yield at the beginning of each period (Danes et al., 2013). The concentrate meal was composed of fine ground corn (80\%), soybean meal $(15 \%)$ and mineral $(5 \%)$, with chemical composition as following: $86.8 \%$ of DM, $9.4 \%$ of ash, $13.6 \%$ of CP, $13.2 \%$ of NDF, $3.4 \%$ of $\mathrm{ADF}, 3.9 \%$ of EE and $59.9 \%$ of NFC.

\subsubsection{Animal measurements}

Cows were weighed and BCS recorded at the end of each sampling period $\left(\mathrm{P}_{1}\right.$ and $\left.\mathrm{P}_{2}\right)$ during three consecutive days (Edmonson et al., 1989). Milk yield was recorded daily with samples collected in vials containing bronopol preservative pill and analyzed for fat, protein, lactose, milk solids and milk urea nitrogen (MUN) using infrared procedures (MilkoScan FT+; Foss North America Inc., Eden Prairie, MN). 
Herbage intake was estimated from total fecal excretion and feed indigestibility. To estimate total fecal excretion, titanium dioxide $\left(\mathrm{TiO}_{2}\right)$ was dosed twice daily $(20 \mathrm{~g} / \mathrm{cow}$ per day) after concentrate meals during 12 days. Fecal samples were collected from rectum following concentrate meals during the last 5 days, dried in a forced-air drier at $55{ }^{\circ} \mathrm{C}$ for $72 \mathrm{~h}$, ground through a 1-mm screen (Wiley Mill, Thomas Scientific, Philadelphia, PA), and composited into one sample per measurement period by cow. Titanium dioxide concentration in feces was determined according to Myers et al. (2004). To determine the feed indigestibility, the indigestible NDF (iNDF) content of herbage, concentrate, and fecal samples were estimated by $240 \mathrm{~h}$ in vitro incubation (Goeser and Combs, 2009). Total fecal excretion, fecal excretion from concentrate, and herbage intake were calculated according to de Souza et al. (2015).

Enteric $\mathrm{CH}_{4}$ emissions were estimated using sulfur hexafluoride $\left(\mathrm{SF}_{6}\right)$ as tracer gas (Johnson and Johnson, 1995). Pre-calibrated permeation tubes containing $\mathrm{SF}_{6}$ with known release rates (1.48 \pm $0.32 \mathrm{mg} / \mathrm{d}$ ) were placed into the rumen of each cow. Sampling apparatus included a PVC collection canister $(2.3 \mathrm{~L})$, and adjustable halter containing stainless steel capillary tubing and brass connections. Canisters were vacuumed to approximately -13.5 psi using a three-stage vacuum pump (Symbol, Sumaré, SP, Brazil) and Druck DPI 705 digital manometer (GE Druck, South Burlington, VT, EUA) and replaced daily just after the afternoon concentrate meal. Cows were adapted to the sampling apparatus during 7 days prior to collection. Enteric $\mathrm{CH}_{4}$ emissions were measured at 24-hour intervals over 7 consecutive days. Background $\mathrm{SF}_{6}$ and $\mathrm{CH}_{4}$ concentrations were determined using two sampling apparatus placed daily in the field near the grazing herd. Prior to chromatograph determination, canisters were pressurized to $1.3-1.5$ psi with ultrapure nitrogen 5.0, and pressures recorded by Druck DPI 705 digital manometer (GE Druck, South Burlington, VT, EUA) in order to calculate the dilution factor. Methane and $\mathrm{SF}_{6}$ concentrations were determined at the Laboratory of Biogeochemistry and Tracer Gases Analysis (Embrapa Meio Ambiente, Jaguariúna, SP, BRA) using gas chromatography (HP6890, Agilent, Delaware, USA). Chromatograph was equipped with flame ionization detector (FID) at $280^{\circ} \mathrm{C}$ for $\mathrm{CH}_{4}$ (column megabore, $0.53 \mathrm{~mm} \times 30 \mathrm{~m} \times 15 \mu \mathrm{m}$, Plot HP$\mathrm{Al} / \mathrm{M}$ ), and electron capture detector $(\mathrm{ECD})$ at $300^{\circ} \mathrm{C}$ for $\mathrm{SF}_{6}$ (column megabore, $0.53 \mathrm{~mm} \times 30 \mathrm{~m} \times$ $25 \mu \mathrm{m}$, HP-MolSiv), with two loops of $0.5 \mathrm{~cm}^{3}$ maintained at $80{ }^{\circ} \mathrm{C}$ attached to two six-way valves. Calibration curves were established using standard certified gases for $\mathrm{CH}_{4}(4.85 \pm 5 \%$; $9.96 \pm 1.65 \%$ and $19.1 \pm 3.44 \% \mathrm{ppm})$ and $\mathrm{SF}_{6}(34.0 \pm 9.0 ; 91.0 \pm 9.0$ and $978.0 \pm 98.0 \mathrm{ppt})$ (Westberg et al., 1998). Daily methane emissions were calculated from collected $\mathrm{SF}_{6}$ and $\mathrm{CH}_{4}$ concentrations in the canisters discounting background concentrations, and value of $\mathrm{SF}_{6}$ permeation tube release rate (Johnson and Johnson, 1995). 


\subsubsection{Statistical analysis}

Analysis of variance was performed using the Mixed Procedure (SAS 9.3; SAS Institute Inc., Cary, NC). Different structures of the variance-covariance matrices were tested and Bayesian Information Criterion was adopted to select the best fit matrix. For plant parameters analysis, paddock was considered as experimental unit, and for animal measurements, cow was considered as experimental unit. Cows or paddocks blocks were considered random terms, and timing of new paddock allocation, sampling period and their interactions were treated as fixed effects. Sampling periods were treated as repeated measures. Means were calculated using the LSMEANS statement and compared using the Student's $t$-test. Differences were declared significant at $P \leq 0.05$, and trends were declared at $P \leq 0.10$.

\subsection{Results}

\subsubsection{Sward characteristics}

Sward characteristics are presented in Table 1. Both pre- and post-grazing SSH did not vary between treatments $(P=0.3124$ and $P=0.8619$, respectively). Post-grazing SSH was greater during $\mathrm{P}_{1}$ than during $\mathrm{P}_{2}(P=0.0127 ; 56.8$ vs. $53.2 \mathrm{~cm}$, respectively). There was no effect of timing of paddock allocation on pre-grazing herbage mass $(P=0.6742)$, leaf-to-stem ratio $(P=0.9214)$ and herbage allowance $(P=0.7694)$.

Table 1. Pre- and post-sward surface height $(\mathrm{SSH})(\mathrm{cm})$, pre-grazing herbage mass ( $\mathrm{kg}$ of $\mathrm{DM} / \mathrm{ha}$ ), leaf:stem ratio and herbage allowance ( $\mathrm{kg}$ of $\mathrm{DM} / \mathrm{cow}$. day) of rotationally managed elephant grass cv. Cameroon with new paddocks allocated to dairy cows either in the morning (AM) or in the afternoon (PM) $(\mathrm{n}=8)$

\begin{tabular}{|c|c|c|c|c|c|c|}
\hline \multirow{2}{*}{ Item } & \multicolumn{2}{|c|}{ Treatments } & \multirow{2}{*}{ SEM $^{1}$} & \multicolumn{3}{|c|}{$P$-value } \\
\hline & $\mathbf{A M}$ & $\mathbf{P M}$ & & Trt $^{2}$ & Per $^{3}$ & Trt $\times$ Per \\
\hline Pre-SSH & 101.6 & 100.4 & 0.80 & 0.2770 & 0.5879 & 0.7848 \\
\hline Post-SSH & 55.7 & 54.2 & 1.18 & 0.2945 & 0.0127 & 0.9889 \\
\hline Pre-grazing herbage mass ${ }^{4}$ & 2270 & 2180 & 208.5 & 0.6742 & 0.6812 & 0.8185 \\
\hline Leaf:Stem ratio ${ }^{4}$ & 73.6 & 75.5 & 30.50 & 0.9214 & 0.9566 & 0.1092 \\
\hline Herbage allowance ${ }^{4}$ & 15.4 & 14.9 & 1.33 & 0.7694 & 0.8090 & 0.4176 \\
\hline
\end{tabular}

\subsubsection{Herbage chemical composition}

Overall, herbage chemical composition differed between treatments (Table 2). Herbage sampled in the afternoon had greater DM $(P=0.0003), \mathrm{SC}(P<0.01)$, starch $(P<0.01), \operatorname{NDICP}(P=$ 
0.0102), NFC/PROT ratio $(P<0.01)$, and lesser $\mathrm{NDF}(P=0.0127)$ and $\mathrm{ADF}(P=0.0053)$ concentrations. There was no treatment effect on OM $(P=0.7879)$, lignin $(P=0.8951)$, EE $(P=$ $0.6610), \mathrm{CP}(P=0.3324)$, soluble and degradable protein $(P=0.2106$ and $P=0.7746$, respectively $)$, and ADICP $(P=0.7893)$ concentrations. During $\mathrm{P}_{2}, \mathrm{DM}(P=0.0007), \mathrm{OM}(P<0.0001), \mathrm{NDF}(P<$ $0.0001)$ and NDICP concentrations $(P<0.0001)$ were greater, and EE $(P=0.0216)$ and degradable protein $(P=0.0064)$ concentrations were lower relative to $P_{1}$. There were no interactions between treatments and sampling periods.

Table 2. Herbage chemical composition (\% of DM) of rotationally managed elephant grass cv. Cameroon with new paddocks allocated to dairy cows either in the morning $(\mathrm{AM})$ or in the afternoon $(\mathrm{PM})(\mathrm{n}=7)$

\begin{tabular}{|c|c|c|c|c|c|c|c|}
\hline \multirow[t]{2}{*}{ Item } & \multicolumn{2}{|c|}{ Treatments } & \multirow[t]{2}{*}{ SEM $^{1}$} & \multirow[t]{2}{*}{$P$-value } & \multicolumn{2}{|c|}{ Periods } & \multirow[t]{2}{*}{$P$-value } \\
\hline & $\mathbf{A M}$ & PM & & & 1 & 2 & \\
\hline Dry matter & 18.9 & 22.2 & 0.67 & 0.0003 & 19.1 & 22.1 & 0.0007 \\
\hline Organic matter & 90.5 & 90.6 & 0.42 & 0.7879 & 89.1 & 92.0 & $<0.0001$ \\
\hline Soluble carbohydrates & 5.4 & 8.2 & 0.27 & $<0.0001$ & 6.8 & 6.8 & 0.8955 \\
\hline Starch & 1.5 & 2.9 & 0.13 & $<0.0001$ & 2.3 & 2.1 & 0.1571 \\
\hline Neutral detergent fiber & 61.8 & 60.0 & 0.56 & 0.0127 & 59.2 & 62.5 & $<0.0001$ \\
\hline Acid detergent fiber & 36.8 & 34.5 & 0.54 & 0.0053 & 35.7 & 35.6 & 0.9287 \\
\hline Lignin & 3.4 & 3.4 & 0.16 & 0.8951 & 3.5 & 3.2 & 0.1237 \\
\hline Ether extract & 3.1 & 3.0 & 0.08 & 0.6610 & 3.1 & 2.9 & 0.0216 \\
\hline Crude protein & 17.6 & 17.1 & 0.42 & 0.3324 & 17.7 & 17.0 & 0.2506 \\
\hline \multicolumn{8}{|c|}{ Protein fractions $^{2}, \%$ of $\mathrm{CP}$} \\
\hline Soluble protein & 26.0 & 24.0 & 1.11 & 0.2106 & 23.9 & 26.1 & 0.1869 \\
\hline Degradable protein & 51.9 & 51.5 & 1.00 & 0.7746 & 53.8 & 49.5 & 0.0064 \\
\hline NDICP & 16.0 & 17.5 & 0.37 & 0.0102 & 15.5 & 18.0 & $<0.0001$ \\
\hline ADICP & 6.6 & 6.6 & 0.31 & 0.7893 & 6.7 & 6.4 & 0.4115 \\
\hline${\mathrm{NFC} / \mathrm{PROT}^{3}}$ & 0.52 & 0.87 & 0.040 & $<0.0001$ & 0.68 & 0.70 & 0.6847 \\
\hline
\end{tabular}

\subsubsection{Animal performance}

The effects of timing of paddock allocation on animal performance are shown in Table 3. There was no treatment effect on milk yield $(P=0.6618)$, fat yield $(P=0.9181)$, and milk solids yield $(P=0.9240)$. However, protein $(P=0.0899)$ and casein $(P=0.0632)$ yields tended to be greater for PM than AM. Timing of paddock allocation did not affect milk fat $(P=0.6285)$, milk protein $(P=$ 0.2976), milk casein $(P=0.2346)$, and milk solids $(P=0.6760)$ concentration. Milk lactose concentration $(P=0.0003)$ and MUN $(P=0.0032)$ were greater for cows grazing paddocks allocated during the morning relative to those allocated in the afternoon. 
Table 3. Milk yield $(\mathrm{kg} / \mathrm{d})$ and milk composition (\% unless specified otherwise) of dairy cows grazing rotationally managed elephant grass cv. Cameroon with new paddocks allocated either in the morning $(\mathrm{AM})$ or in the afternoon $(\mathrm{PM})(\mathrm{n}=10)$

\begin{tabular}{|c|c|c|c|c|c|c|}
\hline \multirow[t]{2}{*}{ Item } & \multicolumn{2}{|c|}{ Treatments } & \multirow[t]{2}{*}{ SEM $^{1}$} & \multicolumn{3}{|c|}{$P$-value } \\
\hline & AM & PM & & Trt $^{2}$ & Per $^{3}$ & Trt $\times$ Per \\
\hline \multicolumn{7}{|l|}{ Yield } \\
\hline Milk & 17.2 & 17.4 & 1.20 & 0.6618 & 0.1023 & 0.8792 \\
\hline Fat & 0.59 & 0.60 & 0.03 & 0.9181 & 0.2626 & 0.5564 \\
\hline Protein & 0.55 & 0.58 & 0.02 & 0.0899 & 0.1802 & 0.3715 \\
\hline Casein & 0.42 & 0.45 & 0.02 & 0.0632 & 0.1021 & 0.0979 \\
\hline Milk solids & 2.1 & 2.1 & 0.11 & 0.9240 & 0.0422 & 0.8164 \\
\hline \multicolumn{7}{|l|}{ Composition } \\
\hline Fat & 3.5 & 3.5 & 0.13 & 0.6285 & 0.9415 & 0.2179 \\
\hline Protein & 3.2 & 3.3 & 0.10 & 0.2976 & 0.0373 & 0.1190 \\
\hline Lactose & 4.6 & 4.4 & 0.06 & 0.0003 & 0.1067 & 0.1627 \\
\hline Casein & 2.5 & 2.6 & 0.09 & 0.2346 & 0.1945 & 0.0295 \\
\hline Milk solids & 12.3 & 12.2 & 0.25 & 0.6760 & 0.3864 & 0.8089 \\
\hline $\mathrm{MUN}^{4}, \mathrm{mg} / \mathrm{dL}$ & 14.6 & 13.0 & 0.46 & 0.0032 & 0.0538 & 0.217 \\
\hline
\end{tabular}

\subsubsection{Dry matter intake and enteric $\mathrm{CH}_{4}$ emissions}

The effects of timing of paddock allocation on DMI and $\mathrm{CH}_{4}$ emissions are shown in Table 4. Herbage DMI $(P=0.97)$ and total DMI $(P=0.9578)$ did not differ between AM and PM treatments. There was no treatment effect on daily enteric $\mathrm{CH}_{4}$ emission $(P=0.9350)$, and efficiencies of milk ( $P$ $=0.6599)$, fat $(P=0.5750)$, protein $(P=0.3070)$, and milk solids $(P=0.6313)$ yield per $\mathrm{g}$ of $\mathrm{CH}_{4}$ emitted. Additionally, timing of paddock allocation did not affect $\mathrm{CH}_{4}$ yield $\left(\mathrm{CH}_{4} / \mathrm{kg}\right.$ DMI; $P=$ $0.3380)$

Table 4. Daily dry matter intake (DMI) (kg of DM/cow) and enteric $\mathrm{CH}_{4}$ emissions of dairy cows grazing rotationally managed elephant grass cv. Cameroon with new paddocks allocated to dairy cows either in the morning $(\mathrm{AM})$ or in the afternoon (PM) $(\mathrm{n}=10)$

\begin{tabular}{|c|c|c|c|c|c|c|}
\hline \multirow[t]{2}{*}{ Item } & \multicolumn{2}{|c|}{ Treatments } & \multirow[t]{2}{*}{ SEM $^{1}$} & \multicolumn{3}{|c|}{$P$-value } \\
\hline & $\mathbf{A M}$ & PM & & Trt $^{2}$ & Per $^{3}$ & Trt $\times$ Per \\
\hline \multicolumn{7}{|l|}{ Daily DMI } \\
\hline Herbage & 11.6 & 11.6 & 0.3862 & 0.97 & 0.27 & 0.002 \\
\hline Total & 17.3 & 17.3 & 0.4494 & 0.9578 & 0.5276 & 0.0033 \\
\hline \multicolumn{7}{|l|}{$\mathrm{CH}_{4}$ emissions } \\
\hline $\mathrm{g} / \mathrm{d}$ & 307.9 & 309.4 & 15.35 & 0.9350 & 0.2848 & 0.5405 \\
\hline $\mathrm{g} / \mathrm{kg}$ of milk yield & 18.8 & 18.3 & 1.48 & 0.6599 & 0.7323 & 0.4390 \\
\hline $\mathrm{g} / \mathrm{kg}$ of fat yield & 543.2 & 516.7 & 39.15 & 0.5750 & 0.9411 & 0.6236 \\
\hline $\mathrm{g} / \mathrm{kg}$ of protein yield & 585.7 & 543.8 & 39.98 & 0.3070 & 0.6489 & 0.2556 \\
\hline $\mathrm{g} / \mathrm{kg}$ of milk solids yield & 154.4 & 148.8 & 11.50 & 0.6313 & 0.9790 & 0.3510 \\
\hline $\mathrm{g} / \mathrm{kg}$ of DMI & 17.3 & 18.5 & 1.23 & 0.3380 & 0.1543 & 0.8623 \\
\hline
\end{tabular}




\subsection{Discussion}

Sward structure is defined as the distribution and arrangement of above-ground plant-part components (Laca and Lemaire, 2000). In tropical grasses, characteristics such as pre- and postgrazing SSH and leaf-to-stem ratio of the pre-grazing herbage mass play, along with herbage allowance, an important role in determining herbage intake and animal performance (Da Silva and Carvalho, 2005; Carvalho, 2013; Congio et al., 2018). In the present study, sward structure characteristics and herbage allowance were similar for both treatments, excluding their effects on other evaluated responses.

Diurnal variations in herbage chemical composition are directly related to NFC accumulation as result of the balance between leaf photosynthesis and plant transpiration (Griggs et al., 2005; Gregorini, 2012; Vibart et al., 2017). In the present study, DM concentration increased by $18 \%$ from AM to PM herbage. Orr et al. (1997) reported increases of $57.3 \%$ and $44.4 \%$ for perennial ryegrass (Lolium perenne L.) and white clover (Trifolium repens L.), respectively. However, most literature reported increases from 14 up to 27\% (Ciavarella et al., 2000; Delagarde et al., 2000; Trevaskis et al., 2001; Gregorini et al., 2008; Abrahamse et al., 2009; De Oliveira et al., 2014; Pulido et al., 2015; Vibart et al., 2017). Diurnal changes in temperature, solar radiation, and relative humidity, coupled with accumulation of photosynthates explain the DM concentration from the morning to the afternoon period (Gregorini et al., 2009).

Several studies described the pattern of NFC accumulation during the day, mostly on temperate swards (Lechtenberg et al., 1971; Orr et al., 1997; Ciavarella et al., 2000; Griggs et al., 2005; Gregorini et al., 2006; Shewmaker et al., 2006; Gregorini et al., 2008; Morin et al., 2011). Greatest concentrations of SC and starch in plants growing in temperate regions were reported between 12-13h after sunrise (Lechtenberg et al., 1971; Morin et al., 2011; Morin et al., 2012; De Oliveira et al., 2018). In our study, afternoon herbage samples were taken approximately $10 \mathrm{~h}$ after sunrise (4 pm), with increases of 52\% in SC and 93\% in starch for PM herbage compared to AM herbage. Greater increases of SC were found in tropical grasses (mean of 68\%; Trevaskis et al., 2001; Fisher et al., 2005; De Oliveira et al., 2014). For temperate swards, including grass and legumes, Pelletier et al. (2010) reported increases of SC from 6 to 105\% for PM herbage compared to AM herbage; however, most results reported mean increases of around 50\% (Ciavarella et al., 2000; Mayland et al., 2000; Pelletier et al., 2010; Vasta et al., 2012; Pulido et al., 2015; Vibart et al., 2017). Increases in starch have been reported around $100 \%$ for PM temperate forage legumes (Orr et al., 1997; Brito et al., 2008; Pelletier et al., 2010; Andueza et al., 2012) and 30\% for PM temperate forage grasses (Orr et al., 1997; Bertrand et al., 2008; Pelletier et al., 2010; Brito et al., 2016).

The increase in NFC and DM during the day dilutes other nutritional entities such as NDF, ADF and CP (Gregorini, 2012; Vibart et al., 2017). In the present study, PM herbage had decreased $\mathrm{NDF}(-2.9 \%)$ and $\mathrm{ADF}(-6.3 \%)$ relative to AM herbage but there was no effect in CP. Burns et al. 
(2007) reported decrease of 7.4 and $6.7 \%$ for NDF and ADF, respectively, and no effect in CP concentration for PM alfalfa (Medicago sativa L.). Similar results were found in perennial ryegrass by Orr et al. (2001) and Abrahamse et al. (2009). Considering CP and N fractions, studies reported a decrease on PM compared to AM herbage (De Oliveira et al., 2014; Pulido et al., 2015; Vibart et al., 2017) while others showed no effect (Delagarde et al., 2000; Fisher et al., 2002; Gregorini et al., 2008). In fact, greater concentrations of SC and starch in the afternoon improve the NFC/PROT ratio which would optimize the supply of energy and protein to rumen microorganisms (Bryant et al., 2012; Bryant et al., 2014) reducing urinary-N excretion and losses onto pastures (Gregorini et al., 2010; Gregorini, 2012; Vibart et al., 2017).

The sampling period effect observed for some herbage chemical composition parameters might be explained by the post-grazing SSH. During $\mathrm{P}_{2}$, post-grazing $\mathrm{SSH}$ was $3.6 \mathrm{~cm}$ lower than during $\mathrm{P}_{1}$, which resulted in slightly greater proportion of stems on the pre-grazing herbage mass (1.9\% for $\mathrm{P}_{2}$ and $0.6 \%$ for $\mathrm{P}_{1} ; P=0.037$ ). Stems contain greater proportion of cell wall and less photosynthetic tissues than leaves (Wilson and Kennedy, 1996) which explains the greater DM, NDF, NDICP, and lower digestible protein reported during the $\mathrm{P}_{2}$. On the other hand, greatest lipid content in plants is found within the chloroplasts (Harwood, 1980), most present in leaves relative to stems.

Daily herbage intake was similar between treatments, which is in agreement with previously reported findings for grazing dairy cows (Gibb et al., 1998; Orr et al., 2001; Abrahamse et al., 2009; Mattiauda et al., 2013; Pulido et al., 2015; Vibart et al., 2017). On the other hand, studies that compared AM and PM herbage for housed ruminants reported greater DMI for animals fed with feedstuffs harvested at sundown (Fisher et al., 1999; Burns et al., 2007; Pagano et al., 2011; Andueza et al., 2012; Brito et al., 2008; 2009; 2016). Herbage DMI of grazing animals is a complex process strongly influenced by non-nutritional or behavioral factors such as sward structure and foraging behavior, whilst for housed animals herbage chemical composition and digestibility seem to be more relevant in setting DMI (Poppi et al., 1987; Hodgson, 1990; Da Silva and Carvalho, 2005; Carvalho, 2013).

Milk yield from dairy cows grazing new paddocks allocated either in the morning or in the afternoon showed only trends rather than significant treatment effects. Orr et al. (2001), although noticing a trend $(P=0.076)$ of $5 \%$ increase in milk yield for PM cows over 4 experimental weeks, concluded that there was no effect during the entire experimental period. Abrahamse et al. (2009) reported significant increase $(P<0.05)$ in fat and protein corrected milk yield and fat yield, even though no differences in milk yield were observed. Mattiauda et al. (2013), restricting grazing time to 4 hours of both periods of paddock allocation, observed a significant increase $(P<0.05)$ in protein yield for PM cows. Pulido et al. (2015) reported no differences in milk and components yields. Recently, Vibart et al. (2017) reported trends $(P<0.10)$ of greater fat, protein, and milk solids yield for PM cows. In this study trends for greater protein $(P=0.0899)$ and casein $(P=0.0632)$ yields were detected for cows grazing PM herbage. Brito et al. (2016) explained that greater proportion of 
supplements may dilute the effect of high NFC of PM herbage. In the present study, concentrate meals represented on average 33\% of total DMI, greater than the amounts used by Vibart et al. (2017) (no concentrate), Orr et al. (2001) (22\%), and Abrahamse et al. (2009) (17\%), and lower than Mattiauda et al. (2013) (62\%) and Pulido et al. (2015) (43\%).

Indoor studies have shown that herbage intake of more balanced fermentable carbon to nitrogen ratio from PM herbage can improve $\mathrm{N}$ utilization of dairy cows (Brito et al., 2008; 2009; 2016). The authors reported lower $\mathrm{N}$ intake, urinary- $\mathrm{N}$ concentration, $\mathrm{N}$ excretion, and more $\mathrm{N}$ partitioning, with greater milk and protein yields for cows eating PM herbage. They also reported lower MUN for PM cows indicating that an improved balance in the supply of energy from NFC and $\mathrm{N}$ can reduce the excretion of urea $\mathrm{N}$ in milk. For grazing dairy cows, Vibart et al. (2017) observed trends of greater $\mathrm{N}$ use efficiency with moderate increases in $\mathrm{N}$ captured towards milk. In this experiment the higher NFC/PROT ratio in the PM herbage reduced the excretion of urea in milk and increased $\mathrm{N}$ into protein and casein yield. This simple and non-cost grazing management strategy can be an useful tool to improve $\mathrm{N}$ efficiency use of dairy cows and reduce $\mathrm{N}$ environment footprint in dairy farming systems.

Enteric $\mathrm{CH}_{4}$ is influenced by the amount and nature of feed ingested by ruminants (Janssen, 2010). In this study, although timing of new paddock allocation has markedly affected herbage chemical composition, it did not affect DMI. Factors that increase passage rate (i.e. higher nutritive value and DMI) decrease $\mathrm{CH}_{4}$ formation per unit of feed eaten (Blaxter and Clapperton, 1965; Janssen, 2010; Hammond et al., 2013). The model proposed by Janssen (2010) suggests that greater passage rates increase hydrogen concentration in the rumen making microorganisms select pathways that produce less hydrogen, resulting in less $\mathrm{CH}_{4} / \mathrm{kg}$ of DM ingested. However, Hammond et al. (2013) reported that 0.85 and 0.87 of total variation in daily enteric $\mathrm{CH}_{4}$ emissions of grazing sheep were predicted by DM and OM intakes, respectively; while herbage chemical composition showed weak correlation with both daily $\mathrm{CH}_{4}$ emission and $\mathrm{CH}_{4}$ yield (g/kg DMI). Although Ellis et al. (2012), in a modeling exercise, showed the possibility to reduce enteric $\mathrm{CH}_{4}$ emission intensity of dairy cows fed with high-sugar grasses, it was not confirmed by the results from this field experiment.

\subsection{Conclusions}

The results ratify the general understanding of diurnal variation in herbage chemical composition towards greater concentrations of NFC and DM, and lower concentration of fiber components in the afternoon herbage. However, the increase in nutritive value of the afternoon relative to the morning herbage was not enough to increase DMI and milk yield, or to decrease $\mathrm{CH}_{4}$ emission intensity by the dairy cows as hypothesized. The findings also indicate that new paddock allocation 
during the afternoon can be a simple and useful grazing strategy that results in greater $\mathrm{N}$ partitioning to protein yield, and lower excretion of urea $\mathrm{N}$ in milk.

\section{References}

Abrahamse, P.A., Tamminga, S., Dijkstra, J., 2009. Effect of daily movement of dairy cattle to fresh grass in morning or afternoon on intake, grazing behaviour, rumen fermentation and milk production. J. Agr. Sci. 147:721-730. https://doi.org/10.1017/S0021859609990153.

Aguirre-Villegas, H.A., Passos-Fonseca, T.H., Reinemann, D.J., Larson, R.A., 2017. Grazing intensity affects the environmental impact of dairy systems. J. Dairy Sci. 100:6804-6821. https://doi.org/10.3168/jds.2016-12325.

Alexandratos, N., Bruinsma, J., 2012. World Agriculture Towards 2030/2050. FAO, Rome http://www.fao.org/fileadmin/templates/esa/Global_persepctives/world_ag_2030_50_2012_rev.pd f.

Andueza, D., Delgado, I., Muñoz, F., 2012. Variation of digestibility and intake by sheep of lucerne (Medicago sativa L.) hays cut at sunrise or sunset. J. Agr. Sci. 150(2):263-270. https://doi.org/10.1017/S0021859611000542.

AOAC International. 2005. Official Methods of Analysis. 18th ed. AOAC International, Gaithersburg, MD.

Bertrand, A., Tremblay, G.F., Pelletier, S., Castonguay, Y., Bélanger, G., 2008. Yield and nutritive value of timothy as affected by temperature, photoperiod and time of harvest. Grass Forage Sci. 63:421-432. https://doi.org/10.1111/j.1365-2494.2008.00649.x.

Blaxter, K.L., Clapperton, J.L., 1965. Prediction of the amount of methane produced by ruminants. Br. J. Nutr. 19, 511-522. https://doi.org/10.1079/BJN19650046.

Brito, A.F., Tremblay, G.F., Bertrand, A., Castonguay, Y., Bélanger, G., Michaud, R., Lafrenière, C., Martineau, R., Berthiaume, R., 2014. Alfalfa baleage with increased concentration of nonstructural carbohydrates supplemented with a corn-based concentrate did not improve production and nitrogen utilization in early lactation dairy cows. J. Dairy Sci. 97:6970-6990. https://doi.org/10.3168/jds.2013-7305.

Brito, A.F., Tremblay, G.F., Bertrand, A., Castonquay, Y., Bélanger, G., Michaud, R., Lafrenière, C., Martineau, R., Berthiaume, R., 2016. Performance and nitrogen use efficiency in mid-lactation dairy cows fed timothy cut in the afternoon or morning. J. Dairy Sci. 99:1-16. https://doi.org/10.3168/jds.2015-10597. 
Brito, A.F., Tremblay, G.F., Lapierre, H., Bertrand, A., Castonguay, Y., Belanger, G., Michaud, R., Benchaar, C., Ouellet, D.R., Berthiaume, R., 2009. Alfalfa cut at sundown increases bacterial protein synthesis in late-lactation dairy cows. J. Dairy Sci. 92:1092-1107. https://doi.org/10.3168/jds.2008-1469.

Brito, A.F., Tremblay, G.F., Lapierre, H., Bertrand, A., Castonquay, Y., Belanger, G., Benchaar, C., Oullet, D.R., Berthiaume, R., 2008. Alfalfa cut at sundown and harvested as baleage improves milk yield of late-lactation dairy cows. J. Dairy Sci. 91:3968-3982. https://doi.org/10.3168/jds.2008-1282.

Bryant, R.H., Dalley, D.E., Gibbs, J., Edwards, G.R., 2014. Effect of grazing management on herbage protein concentration, milk production and nitrogen excretion of dairy cows in mid-lactation. Grass Forage Sci. 69:644-654. https://doi.org/10.1111/gfs.12088.

Bryant, R.H., Gregorini, P., Edwards, G.R., 2012. Effects of N fertilisation, leaf appearance and time of day on $\mathrm{N}$ fractionation and chemical composition of Lolium perenne cultivars in spring. Anim. Feed Sci. Technol. 173:210-219. https://doi.org/10.1016/j.anifeedsci.2012.02.003.

Burns, J.C., Fischer, D.S., Mayland, H.F., 2007. Diurnal shifts in nutritive value of alfalfa harvested as hay and evaluated by animal intake and digestion. Crop Sci. 47:2190-2197. https://doi.org/10.2135/cropsci2007.02.0072.

Carvalho, P.C.F., 2013. Harry Stobbs memorial lecture: can grazing behavior support innovations in grassland management? Trop. Grassl.-Forrajes Trop. 1:137-155. https://doi.org/10.17138/TGFT(1)137-155.

CEPAGRI, 2012. Centro de pesquisas meteorológicas e climáticas aplicadas à agricultura. [Center of Applied Climatic and Meteorological Research in Agriculture]. UNICAMP, Campinas. http://www.cpa.unicamp.br/outras-informacoes/clima_muni_436.html.

Chapman, D., 2016. Using ecophysiology to improve farm efficiency: application in temperate dairy grazing systems. Agriculture 6:1-19. https://doi.org/10.3390/agriculture6020017.

Chilibroste, P., Soca, P., Mattiauda, D.A., Bentancur, O., Robinson, P.H., 2007. Short-term fasting as a tool to design effective grazing strategies for lactating dairy cattle: a review. Aust. J. Exp. Agric. 47:1075-1084. https://doi.org/10.1071/EA06130.

Ciavarella, T.A., Simpson, R.J., Dove, H., Leyry, B.J., Sims, I.M., 2000. Diurnal differences in the concentration of water-soluble carbohydrates in Phalaris aquatica L. pasture in spring, and the effect of short-term shading. Aust. J. Agric. Res. 51:749-756. https://doi.org/10.1071/AR99150.

Conforti, P., 2011. Looking Ahead in World Food and Agriculture: Perspectives to 2050. Food and Agriculture Organization, Rome. http://www.fao.org/docrep/014/i2280e/i2280e.pdf.

Congio, G.F.S., Batalha, C.D.A., Chiavegato, M.B., Berndt, A., Oliveira, P.P.A., Frighetto, R.T.S., Maxwell, T.M.R., Gregorini, P., Da Silva, S.C., 2018. Strategic grazing management towards sustainable intensification at tropical pasture-based dairy systems. Sci. Total Environ. 636:872880. https://doi.org/10.1016/j.scitotenv.2018.04.301. 
Crosson, P., Shalloo, L., O’Brien, D., Lanigan, G.J., Foley, P.A., Boland, T.M., Kenny, D.A., 2011. A review of whole farm systems models of greenhouse gas emissions from beef and dairy cattle production systems. Anim. Feed Sci. Technol. 166-167:29-45. https://doi.org/10.1016/j.anifeedsci.2011.04.001.

Curtis, O.F. 1944. The food content of forage crops as influenced by the time of day at which they were cut. J. Agron. 36:401-416.

Da Silva, S.C., Carvalho, P.C.F., 2005. Foraging behaviour and herbage intake in the favourable tropics/subtropics. In: McGilloway, D.A. (Ed.), Grassland: A Global Resource. Wageningen Academic Publishers, Wageningen, The Netherlands :pp. 81-96. http://citeseerx.ist.psu.edu/viewdoc/download?doi=10.1.1.511.4117\&rep=rep1\&type=pdf.

Da Silva, S.C., Sbrissia, A.F., Pereira, L.E.T., 2015. Ecophysiology of C4 forage grassesunderstanding plant growth for optimising their use and management. Agriculture 5:598-625. https://doi.org/10.3390/agriculture5030598.

Danes, M.A., Chagas, L.J., Pedroso, A.M., Santos, F.A.P., 2013. Effect of protein supplementation on milk production and metabolism of dairy cows grazing tropical grass. J. Dairy Sci. 96:407-419. https://doi.org/10.3168/jds.2012-5607.

De Oliveira, F.C.L., Sanchez, J.M.D., Vendramini, J.M.B., Lima, C.G., Luz, P.H.C., Rocha, C.O., Pereira, L.E.T., Herling, V.R., 2018. Diurnal vertical and seasonal changes in non-structural carbohydrates in Marandu palisade grass. J. Agric. Sci. 1-8. https://doi.org/10.1017/ $\underline{\text { S0021859618000394. }}$

De Oliveira, L.P., Paiva, A., Pereira, L.E.T., Geremia, E.V., Da Silva, S.C., 2014. Morning and afternoon sampling and herbage chemical composition of rotationally stocked elephant grass cv. Napier. Trop. Grassl.-Forrajes Trop. 2:106-107. https://doi.org/10.17138/tgft(2)106-107.

De Souza, J., Batistel, F., Santos, F.A.P., 2017. Effect of sources of Ca salts of fatty acids on production, nutrient digestibility, energy balance, and carryover effects of early lactation grazing dairy cows. J. Dairy Sci. 100:1072-1085. https://doi.org/10.3168/jds.2016-11636.

De Souza, J., Batistel, F., Welter, K.C., Silva, M.M.V., Costa, D.F., Santos, F.A.P., 2015. Evaluation of external markers to estimate fecal excretion, intake and digestibility in dairy cows. Trop. Anim. Health Prod. 47: 265-268. https://doi.org/10.1007/s11250-014-0674-6.

Delagarde, R., Peyraud, J.L., Delaby, L., Faverdin, P., 2000. Vertical distribution of biomass, chemical composition and pepsin-cellulase digestibility in a perennial ryegrass sward: Interaction with month of year, regrowth age and time of day. Anim. Feed Sci. Technol. 84:49-68. https://doi.org/10.1016/S0377-8401(00)00114-0.

Edmonson, A.J., Lean, I.J., Weaver, L.D., Farver, T.,Webster, G., 1989. A body condition scoring chart for Holstein dairy cows. J. Dairy Sci. 72:68-78. https://doi.org/10.3168/jds.S00220302(89)79081-0. 
Ellis, J.L., Dijkstra, J., France, J., Parsons, A.J., Edwards, G.R., Rasmussen, S., Kebreab, E., Bannink, A., 2012. Effect of high-sugar grasses on methane emissions simulated using a dynamic model. J. Dairy Sci. 95:272-285. https://doi.org/10.3168/jds.2011-4385.

Enriquez-Hidalgo, D., T. Gilliland, M. H. Deighton, M. O’Donovan, and D. Hennessy., 2014. Milk production and enteric methane emissions by dairy cows grazing fertilized perennial ryegrass pasture with or without inclusion of white clover. J. Dairy Sci. 97:1400-1412. https://doi.org/10.3168/jds.2013-7034.

Fisher, D.S., Burns, J.C., Mayland, H.F., 2005. Ruminant selection among switchgrass hays cut at either sundown or sunup. Crop Sci. 45:1394-1402. https://doi.org/10.2135/cropsci2004.0388.

Fisher, D.S., Mayland, H.F., Burns, J.C., 2002. Variation in ruminant preference for alfalfa hays cut at either sundown or sunup. Crop Sci. 42:231-237. https://doi.org/10.2135/cropsci2002.2310.

Fisher, D.S., Mayland, H.F., Burns, J.C.,1999. Variation in ruminant preference for tall fescue hays cut at sundown or sunup. J. Anim. Sci. 77:762-768. https://doi.org/10.2527/1999.773762x.

Fonseca, L., Mezzalira, J.C., Bremm, C., Filho, R.S.A., Gonda H.L., Carvalho, P.C.F., 2012. Management targets for maximising the short-term herbage intake rate of cattle grazing in Sorghum bicolor. Livest. Sci. 145:205-211. https://doi.org/10.1016/j.livsci.2012.02.003.

Foote, K.J., Joy, M.K., Death, R.G., 2015. New Zealand dairy farming: milking our environment for all its worth. Environ. Manage. 56:709-720. https://doi.org/10.1007/s00267-015-0517-x.

Gibb, M.J., Huckle, C.A., Nuthall, R., 1998. Effect of time of day on grazing behaviour by lactating dairy cows. Grass Forage Sci. 53:41-46. https://doi.org/10.1046/j.1365-2494.1998.00102.x.

Godfray, H., Beddington, J.R., Crute, I.R., Haddad, L., Lawrence, D., Muir, J.F., Pretty, J., Robinson, S., Thomas, S.M., Toulmin, C., 2010. Food security: the challenge of feeding 9 billion people. Science. 327:812-818. https://doi.org/10.1126/science.1185383.

Goeser, J.P., Combs, D.K. 2009. An alternative method to assess 24-h ruminal in vitro neutral detergent fiber digestibility. J. Dairy Sci. 92:3833-3841. http://dx.doi.org/10.3168/jds.2008-1136.

Gregorini, P. 2012. Diurnal grazing pattern: its physiological basis and strategic management. Anim. Prod. Sci. 52:416-430. http://dx.doi.org/10.1071/AN11250.

Gregorini, P., Beukes, P.C., Bryant, R.H., Romera, A.J. 2010. A brief overview and simulation of the effects of some feeding strategies on nitrogen excretion and enteric methane emission from grazing dairy cows. In: Edwards G.R. and Bryant R.H. (eds) Proceedings of the 4th Australasian Dairy Science Symposium. Canterbury, New Zealand: Lincoln University. http://www.sciquest.org.nz/elibrary/download/69329/A+brief+overview+and+simulation+of+the+ $\underline{\text { effects+of+some+feeding+strategies+on+nitrogen+excretion+and+enteric+methane+emission+fro }}$ m+grazing+dairy+cows.

Gregorini, P., Eirin, M., Refi, R., Ursino, M., Ansin, O.E., Gunter, S.A., 2006. Timing of herbage allocation: Effect on beef heifers daily grazing pattern and performance. J. Anim. Sci. 84:19431950. https://doi.org/10.2527/jas.2005-537. 
Gregorini, P., Gunter, S.A., Beck, P.A., 2008. Matching plant and animal processes to alter nutrient supply in strip grazed cattle: timing of herbage and fasting allocation. J. Anim. Sci. 86:1006-1020. https://doi.org/10.2527/jas.2007-0432.

Gregorini, P., Gunter, S.A., Beck, P.A., Soder, K.J., Tamminga, S., 2008. The interaction of diurnal grazing pattern, ruminal metabolism, nutrient supply and management in cattle. Prof. Anim. Sci. 24:308-318. https://doi.org/10.15232/S1080-7446(15)30861-5.

Gregorini, P., Soder, K.J., Sanderson, M.A., Ziegler, G., 2009. Toughness, particle size and chemical composition of meadow fescue (Festuca pratensis Hud.) herbage as affected by time of day. Anim. Feed Sci. Technol. 151:330-336. https://doi.org/10.1016/j.anifeedsci.2009.02.004.

Gregorini, P., Villalba, J.J., Chilibroste, P., Provenza, F.D., 2017. Grazing management: setting the table, designing the menu and influencing the diner. Anim. Prod. Sci. 57(7):1248-1268. http://dx.doi.org/10.1071/AN16637.

Griggs, T.C., MacAdam, J.W., Mayland, H.F., Burns, J.C., 2005. Nonstructural carbohydrate and digestibility patterns in orchardgrass swards during daily defoliation sequences initiated in evening and morning. Crop Sci. 45:1295-1304. https://doi.org/10.2135/cropsci2003.0613.

Guerci, M., Knudsen, M.T., Bava, L., Zucali, M., Schonbach, P., Kristensen, T., 2013. Parameters affecting the environmental impact of a range of dairy farming systems in Denmark, Germany and Italy. J. Clean. Prod. 54:133-141. https://doi.org/10.1016/j.jclepro.2013.04.035.

Hall, M.B., 2003. Challenges with nonfiber carbohydrate methods. J. Anim. Sci. 81:3226-3232. https://doi.org/10.2527/2003.81123226x.

Hammond, K.J., Burke, J.L., Koolaard, J.P., Muetzel, S., Pinares- Patino, C.S., Waghorn, G.C., 2013. Effects of feed intake on enteric methane emissions from sheep fed fresh white clover (Trifolium repens) and perennial ryegrass (Lolium perenne) forages. Anim. Feed Sci. Technol. 179:121-132. https://doi.org/10.1016/j.anifeedsci.2012.11.004.

Harwood, J.L., 1980. Plant acyl lipids: Structure, distribution and analysis. In: Stumpf, P.K. and Conn, E.E. (eds). The Biochemistry of Plants, Vol 4, pp 1-55. Academic Press, New York. https://doi.org/10.1016/B978-0-12-675404-9.50007-2.

Hodgson, J., 1990. Grazing management: science into practice. Ed. Longman Scientific \& Technical. $203 \mathrm{p}$.

Hristov, A.N., Oh, J., Firkins, J.L., Dijkstra, J., Kebreab, E., Waghorn, G., Makkar, H.P., Adesogan, A.T., Yang, W., Lee, C., Gerber, P.J., Henderson, B., Tricarico, J.M., 2013. Special topicsMitigation of the methane and nitrous oxide emissions from animal operations: I. A review of enteric methane mitigation options. J. Anim. Sci. 91:5045-5069. https://doi.org/10.2527/jas.2013$\underline{6583 .}$.

Janssen, P.H., 2010. Influence of hydrogen on rumen methane formation and fermentation balances through microbial growth kinetics and fermentation thermodynamics. Anim. Feed Sci. Technol. 160:1-22. https://doi.org/10.1016/j.anifeedsci.2010.07.002. 
Johnson, K.A., Johnson, D.E., 1995. Methane emissions from cattle. J. Anim. Sci. 73:2483-2492. https://doi.org/10.2527/1995.7382483x.

Laca, E.A., Lemaire, G., 2000. Measuring sward structure. In: Mannetje, L., Jones, R.M. (Eds.), Field and Laboratory Methods for Grassland and Animal Production Research. CABI, Wallingford, UK:pp. 103-121 https://doi.org/10.1079/9780851993515.0000.

Lechtenberg, V.L., Holt, D.A., Youngberg, H.W., 1971. Diurnal variation in nonstructural carbohydrates, in vitro digestibility, and leaf to stem ratio of alfalfa. Agron. J. 63:719-724. https://doi.org/10.2134/agronj1971.00021962006300050019x.

Licitra, G., Hernandez, T.M., Van Soest, P.J., 1996. Standardization of procedures for nitrogen fractionation of ruminant feeds. Anim. Feed Sci. Technol. 57:347-358. https://doi.org/10.1016/0377-8401(95)00837-3.

Macdonald, K.A., Penno, J.W., Lancaster, J.A.S., Bryant, A.M., Kidd, J.M., Roche, J.R., 2017. Production and economic responses to intensification of pasture-based dairy production systems. J. Dairy Sci. 100:6602-6619. https://doi.org/10.3168/jds.2016-12497.

Mattiauda, D.A.; Tamminga, S.; Gibb, M.J.; Soca, P.; Bentancur, O; Chilibroste, P., 2013. Restricting access time at pasture and time of grazing allocation for Holstein dairy cows: Ingestive behaviour, dry matter intake and milk production. Livest. Sci. 152:53-62. https://doi.org/10.1016/j.livsci.2012.12.010.

Mayland, H.F., Shewmaker, G.E., Harrison, P.A., Chatterton, N.J., 2000. Nonstructural carbohydrates in tall fescue cultivars: Relationship to animal preference. Agron. J. 92:1203-1206. https://doi.org/10.2134/agronj2000.9261203x.

Morin, C., Bélanger, G., Tremblay, G.F., Bertrand, A., Castonguay, Y., Drapeau, R., Michaud, R., Berthiaume, R., Allard, G., 2011. Diurnal variations of nonstructural carbohydrates and nutritive value in alfalfa. Crop Sci. 51:1297-1306. https://doi.org/10.2135/cropsci2010.07.0406.

Morin, C., Bélanger, G., Tremblay, G.F., Bertrand, A., Castonguay, Y., Drapeau, R., Michaud, R., Berthiaume, R., Allard, G., 2012. Short Communication: Diurnal variations of nonstructural carbohydrates and nutritive value in timothy. Can. J. Plant Sci. 92:883-887. https://doi.org/10.1139/CJPS2011-272.

Muñoz, C., Letelier, P.A., Ungerfeld, E.M., Morales, J.M., Hube, S., Pérez-Prieto, L.A., 2016. Effects of pre grazing herbage mass in late spring on enteric methane emissions, dry matter intake, and milk production of dairy cows. J. Dairy Sci. 99:7945-7955. https://doi.org/10.3168/jds.2016$\underline{10919 .}$.

Myers, W.D., Ludden, P.A., Nayigihugu, V., Hess, B.W., 2004. Technical note: a procedure for preparation and quantitative analysis of samples for titanium dioxide. J. Anim. Sci. 82:179-193. https://pdfs.semanticscholar.org/4ad3/c1f840f0070e800998507f77c9394f24c631.pdf. 
Orr, R.J., Penning, P.D., Harvey, A., Champion, R.A., 1997. Diurnal patterns of intake rate by sheep grazing monocultures of rye grass or white clover. Appl. Anim. Behav. Sci. 53:65-77. https://doi.org/10.1016/S0168-1591(96)01120-3.

Orr, R.J., Rutter, S.M., Penning, P.D., Rook, A.J., 2001. Matching grass supply to grazing patterns for dairy cows. Grass Forage Sci. 56:352-361. https://doi.org/10.1046/j.1365-2494.2001.00284.x.

Pagano, R.I., Valenti, B., De Angelis, A., Avondo, M., Pennisi, P., 2011. Morning versus afternoon cutting time of Berseem clover (Trifolium alexandrinum L.) affects feed intake, milk yield and composition in Girgentana goats. J. Dairy Res. 78:500-504. https://doi.org/10.1017/S0022029911000719.

Pelletier, S., Tremblay, G.F., Belanger, G., Bertrand, A., Castonguay, Y., Pageau, D., Drapeau, R., 2010. Forage nonstructural carbohydrates and nutritive value as affected by time of cutting and species. Agron. J. 105:1388-1398. https://doi.org/10.2134/agronj2010.0158.

Pereira, L.E.T., Paiva, A.J., Geremia, E.V., Da Silva, S.C., 2014. Components of herbage accumulation in elephant grass cvar Napier subjected to strategies of intermittent stocking management. J. Agr. Sci. 152:954-966. https://doi.org/10.1017/S0021859613000695.

Pereira, L.E.T., Paiva, A.J., Geremia, E.V., Da Silva, S.C., 2015a. Grazing management and tussock distribution in elephant grass. Grass Forage Sci. 70:1-12. https://doi.org/10.1111/gfs.12137.

Pereira, L.E.T., Paiva, A.J., Geremia, E.V., Da Silva, S.C., 2015b. Regrowth patterns of elephant grass (Pennisetum purpureum Schum.) subjected to strategies of intermittent stocking management. Grass Forage Sci. 70:195-204. https://doi.org/10.1111/gfs.12103.

Pérez-Prieto, L., Delagarde, R., 2013. Meta-analysis of the effect of pasture allowance on pasture intake, milk production, and grazing behavior of dairy cows grazing temperate grasslands. J. Dairy Sci. 96:6671-6689. https://doi.org/10.3168/jds.2013-6964.

Poppi, D.P., Hughes, T.P., L'Huillier, P.J., 1987. Intake of pasture by grazing ruminants. In Feeding Livestock on Pasture, Nicol, A.M., Ed., Occasional Publication, New Zealand Society of Animal Production: Hamilton, New Zealand. 55-63.

Pulido, R.G., Ruiz-Albarran, M. Balocchi, O.A., Nannig, P., Wittwer, F., 2015. Effect of timing of pasture allocation on production, behavior, rumen function, and metabolism of early lactating dairy cows during autumn. Livest. Sci. 177:43-51. https://doi.org/10.1016/j.livsci.2015.04.002.

Santos, F.A.P., Dorea, J.R.R., de Souza, J., Batistel, F., Costa, D.F.A., 2014. Forage management and methods to improve nutrient intake in grazing cattle in Proc. 25th Annu. Florida Rumin. Nutr. Symp. pp 144-164. Univ. Florida, Gainesville. http://dairy.ifas.ufl.edu/rns/2014/santos.pdf.

Sauvé, A.K., Huntington, G.B., Whisnant, C.S., Burns, J.C., 2010. Intake, digestibility, and nitrogen balance of steers fed gamagrass baleage top dressed at two rates of nitrogen and harvested at sunset and sunrise. Crop Sci. 50:427-437. https://doi.org/10.2135/cropsci2009.02.0105. 
Shewmaker, G.E., Mayland, H.F.; Roberts, C.A.; Harrison, P.A.; Chatterton, N.J.; Sleper, D.A., 2006. Daily carbohydrate accumulation in eight tall fescue cultivars. Grass Forage Sci. 61:413-421. https://doi.org/10.1111/j.1365-2494.2006.00550.x.

Sniffen, C.J., O’Connor, J.D., Van Soest, P.J., Fox, D.G., Russell, J.B., 1992. A net carbohydrate and protein system for evaluating cattle diets: II. Carbohydrate and protein availability. J. Anim. Sci. 70:3562-3577. https://doi.org/10.2527/1992.70113562x.

Tilman, D., Cassman, K.G., Matson, P.A., Naylor, R., Polasky, S., 2002. Agricultural sustainability and intensive production practices. Nature 418:671-677. https://doi.org/10.1038/nature01014.

Trevaskis, L.M., Fulkerson, W.J., Gooden, M., 2001. Provision of certain carbohydrate-based supplements to pasture-fed sheep, as well as time of harvesting of the pasture, influences $\mathrm{pH}$, ammonia concentration and microbial protein synthesis in the rumen. Aust. J. Exp. Agric. 41:2127. https://doi.org/10.1071/EA00063.

Van Soest, P.J., Robertson, J.B., Lewis, B.A., 1991. Methods for dietary fiber, neutral detergent fiber and nonstarch polysaccharides in relation to animal nutrition. J. Dairy Sci. 74:3583-3597. http://dx.doi.org/10.3168/jds.S0022-0302(91)78551-2.

Vasta, V., Pagano, R,I., Luciano, G., Scerra, M.,Caparra, P., Foti, F., Cilione, C., Biondi, L., Priolo, A., Avondo, M., 2012. Effect of morning vs. afternoon grazing on intramuscular fatty acid composition in lamb. Meat Sci. 90:93-98. http://dx.doi.org/10.1016/j.meatsci.2011.06.009.

Vibart, R.E., Tavendale, M., Otter, D., Schwendel, B.H., Lowe, K., Gregorini, P., Pacheco, D., 2017. Milk production and composition, nitrogen utilization, and grazing behavior of late-lactation dairy cows as affected by time of allocation of a fresh strip of pasture. J. Dairy Sci. 100, 1-14. http://dx.doi.org/10.3168/jds.2016-12413.

Westberg, H.H., Johnson, K.A., Cossalman,M.W.,Michal, J.J., 1998. A SF6 Tracer Technique: Methane Measurement from Ruminants. 2nd ed. Pullman-Washington, Washington State University.

Wilson, J.R., Kennedy, P.M., 1996. Plant and animal constraints to voluntary feed intake associated with fibre characteristics and particle breakdown and passage in ruminants. Aust. J. Agric. Res. 47:199-225. https://doi.org/10.1071/AR9960199.

Wims, C.M., Deighton, M.H., Lewis, E., O'Loughlin, B., Delaby, L., Boland, T.M., O'Donovan, M., 2010. Effect of pregrazing herbage mass on methane production, dry matter intake, and milk production of grazing dairy cows during the mid-season period. J. Dairy Sci. 93:4976-4985. https://doi.org/10.3168/jds.2010-3245.

Yari, M., Valizadeh, R., Naserian, A.A., Jonker, A., Azarfard, A., Yu, P., 2014. Effects of including alfalfa hay cut in the afternoon or morning at three stages of maturity in high concentrate rations on dairy cows performance, diet digestibility and feeding behavior. Anim. Feed Sci. Technol. 192:62-72. https://doi.org/10.1016/j.anifeedsci.2014.04.001. 


\section{GENERAL CONSIDERATIONS}

At the present time, the expected demand for food places farming systems under pressure (Chiavegato et al., 2018), but the increase in agricultural outputs has to be coupled with the decrease in environmental footprint (Godfray et al., 2010; Foley et al., 2011). In developing countries, agricultural production must increase $80 \%$ through higher yields resulting from intensification of existing agricultural systems (Conforti, 2011). In this sense, the concept of sustainable intensification began to be addressed in such systems as a means of achieving higher yields through practices that decrease the impact of key environmental issues (Royal Society, 2009; Garnett and Godfray, 2012).

Dairy farming systems from temperate pastures are more intensive than those from tropical pastures (Congio et al., 2018) and their intensification is usually associated with more inputs of nitrogen, to boost forage growth, or external supplementary feed, both aiming at increasing stocking rate and productivity (Ramsbottom et al., 2015; Macdonald et al., 2017). In the tropics, dairy farming systems besides having low $\mathrm{N}$ inputs, usually adopt inadequate grazing management strategies resulting in low levels of milk productivity. Therefore, the sustainable intensification of tropical pasture-based dairy systems may be possible through adoption of adequate grazing strategies rather than extra nitrogen inputs or additional supplementary feed (Congio et al., 2018), provided that minimum levels of soil fertility are provided to meet plant nutritional requirements.

This study was based in the literature that described the growth pattern of tropical forage grass species under grazing (Carnevalli et al., 2006; Barbosa et al., 2007; Trindade et al., 2007; Da Silva et al., 2009; Difante et al., 2009; Giacomoni et al., 2009; Barbosa et al., 2011; Gimenes et al., 2011; Zanini et al., 2012; Silveira et al., 2013; Geremia et al., 2014; Pereira et al., 2014; Pereira et al., 2015a; Pereira et al., 2015b; Silveira et al., 2016; Da Silva et al., 2017; Pereira et al., 2018; Sbrissia et al., 2018). In general, there is a change in plant growth and pattern of herbage accumulation during regrowth after reaching the canopy critical leaf area index (i.e. $\mathrm{LI}_{95 \%}$ ), when stem elongation and dead material accumulation increase at the expense of leaf accumulation. Further, it has been systematically observed that there is a positive relationship between canopy light interception and sward surface height (SSH), indicating that SSH may be used as a reliable field index for monitoring and controlling herbage regrowth (Da Silva et al., 2015). The results from this study corroborated the greater leaf accumulation, herbage nutritive value, greater grazing efficiency, better tussock distribution, and lower grazing losses on swards managed with the $\mathrm{LI}_{95 \%}$ relative to the $\mathrm{LI}_{\mathrm{Max}}$ pre-grazing target. The results from this study also integrated animal with plant responses and showed that the pattern of plant growth during regrowth when managed with the $\mathrm{LI}_{95 \%}$ target provides benefits to grazing animals and to the system such as greater dry matter intake, higher milk yield and stocking rate resulting in 51\% increase in milk productivity. Additionally, benefits regarding issues of environmental concern were also associated with this grazing management strategy, and corresponded to mitigation of emissions of 
the two most representative greenhouse gases (GHG) in dairy farming systems per $\mathrm{kg}$ of milk produced (i.e. $\mathrm{CH}_{4}$ and $\mathrm{N}_{2} \mathrm{O} / \mathrm{kg}$ of milk).

Once the ideal pre-grazing management target was established during the first experiment, the second experiment aimed at seeking the possibility of refinement by studying the ideal time of the day to move animals to a new paddock. The results indicated that allocation of a new paddock at the right pre-grazing condition during the afternoon provides herbage with a more balanced NFC/PROT ratio to dairy cows, resulting in improved balance of protein and energy supply, and favoring increased $\mathrm{N}$ retention through enhanced milk protein yield and less $\mathrm{N}$ as milk urea nitrogen. The association of the $\mathrm{LI}_{95 \%}$ pre-grazing target and PM allocation could bring economic, productivity and environmental benefits towards sustainable intensification of tropical pasture-based systems. Both findings highlight the opportunity to improve the efficiency of tropical pasture-based dairy systems through practices that decrease the impact of key environmental issues, in accordance with the principles of sustainable intensification.

Recently, land-based research institutes have been concentrating efforts in assessing sources of GHG emissions in a broad range of agricultural systems around the world in order to generate field data that can support accurate carbon footprint reports (Muñoz et al., 2016; Nascimento et al., 2016; Luo et al., 2018; Pontes et al., 2018). Particularly, there are few data available regarding GHG emissions in tropical regions, where most studies estimate carbon footprint from agricultural systems using IPCC data (Intergovernmental Panel on Climate Change), with results that may be not so accurate (Lessa et a., 2014; Cunha et al., 2016). Therefore, in loco studies are mandatory to support accurate carbon footprint reports in tropical climate regions. Other aspect that impairs determination of the real carbon footprint from agricultural systems is that most efforts are towards measuring sources of GHG and few to evaluate carbon sequestration and storage in the soil. Perennial tropical pastures on moist-warm climate have an enormous potential to increase soil organic carbon and offset GHG emissions from livestock pasture-based systems (Braz et al., 2013; Abdalla et al., 2018). Abdalla et al. (2018) highlighted that $\mathrm{C}_{4}$ grass species under high grazing intensities in moist-warm regions are more likely to increase soil organic carbon than $\mathrm{C}_{4}$ under low grazing intensity. Therefore, further research should focus on the analysis of carbon sequestration and stock in the soil, to achieve a more accurate estimate of carbon balance and, therefore, to encourage mitigation strategies and programs by producers in association with companies and policy makers. 


\section{References}

Abdalla, M., Hastings, A., Chadwick, D.R., Jones, D.L., Evans, C.D., Jones, M.B., Rees, R.M., Smith, P., 2018. Critical review of the impacts of grazing intensity on soil organic carbon storage and other soil quality indicators in extensively managed grasslands. Agric. Ecosyst. Environ. 253:6281. https://doi.org/10.1016/j.agee.2017.10.023.

Barbosa, R.A., Nascimento Jr., D., Euclides, V.P.B., Da Silva, S.C., Zimmer, A.H., Torres Jr., R.A.A., 2007. Capim Tanzânia submetido a combinações entre intensidade e frequência de pastejo. Pesq. Agropec. Bras. 42:329-340. https://doi.org/10.1590/S0100-204X2007000300005.

Barbosa, R.A., Nascimento Júnior, D., Vilela, H.H., Da Silva, S.C., Euclides, V.P.B., Sbrissia, A.F., Sousa, B.M.L., 2011. Morphogenic and structural characteristics of guinea grass pastures submitted to three frequencies and two defoliation severities. Rev. Bras. Zootecn. 40 (5):947-954. http://doi.org/10.1590/S1516-35982011000500002.

Braz, S.P., Urquiaga, S., Alves, B.J.R., Jantalia, C.P., Guimarães, A.P., dos Santos, C.A., dos Santos, S.C., Pinheiro, E.F.M., Boddey, R.M., 2013. Soil Carbon Stocks under Productive and Degraded Brachiaria Pastures in the Brazilian Cerrado. Soil Sci. Soc. Am. 77:914-928. https://doi.org/10.2136/sssaj2012.0269.

Carnevalli, R.A., Da Silva, S.C., Bueno, A.A.O., Uebele, M.C., Bueno, F.O., Hodgson, J., Silva, G.N., Morais, J.P.G., 2006. Herbage production and grazing losses in Panicum maximum cv. Mombaça under four grazing management. Trop. Grassl.-Forrajes Trop. 40:165-176. http://tropicalgrasslands.info/public/journals/4/Historic/Tropical\%20Grasslands\%20Journal\%20ar chive/PDFs/Vol_40_2006/Vol_40_03_2006_pp165_176.pdf.

Chiavegato, M.B., Congio, G.F.S., Da Silva, S.C., 2018. Estratégias de manejo do pastejo para redução de impactos ambientais. In: Anais do $4^{\circ}$ Simpósio Brasileiro de Produção de Ruminantes no Cerrado: Eficiência produtiva e impacto ambiental na produção de ruminantes. UFU, Uberlândia, Brasil, pp. 15-36. http://www.eventos.ufu.br/sites/eventos.ufu.br/files/documentos/anais iv simprucerrado versao f inal com resumos.pdf.

Conforti, P., 2011. Looking Ahead in World Food and Agriculture: Perspectives to 2050. Food and Agriculture Organization, Rome. http://www.fao.org/docrep/014/i2280e/i2280e.pdf.

Congio, G.F.S., Batalha, C.D.A., Chiavegato, M.B., Berndt, A., Oliveira, P.P.A., Frighetto, R.T.S., Maxwell, T.M.R., Gregorini, P., Da Silva, S.C., 2018. Strategic grazing management towards sustainable intensification at tropical pasture-based dairy systems. Sci. Total Environ. 636:872880. https://doi.org/10.1016/j.scitotenv.2018.04.301. 
Cunha, C.S.; Lopes, N.L.; Veloso, C.M.; Jacovine, L.A.G.; Tomich, T.R.; Pereira, L.G.R.; Marcondes, M.I., 2016. Greenhouse gases inventory and carbon balance of two dairy systems obtained two $\begin{array}{llll}\text { methane-estimation } & \text { methods. Sci. }\end{array}$ http://dx.doi.org/10.1016/j.scitotenv.2016.07.046.

Da Silva, S.C., Bueno, A.A.O., Carnevalli, R.A., Uebele, M.C., Bueno, F.O., Hodgson, J., Matthew, C., Arnold, J.C., Morais, J.P.G., 2009. Sward structural characteristics and herbage accumulation of Panicum maximum cv. Mombaça subject to rotational stocking managements. Sci. Agric. 66:819. https://doi.org/10.1590/S010390162009000100002.

Da Silva, S.C., Chiavegato, M.B., Pena, K.S., Silveira, M.C.T., Barbero, L.M., Junior, S.J.S., Rodrigues, C.S., Limão, V.A., Pereira, L.E.T., 2017. Tillering dynamics of Mulato grass subjected to strategies of rotational grazing management. J. Agric. Sci. 155:1082-1092. https://doi.org/10.1017/S0021859617000223.

Da Silva, S.C., Sbrissia, A.F., Pereira, L.E.T., 2015. Ecophysiology of C4 forage grassesunderstanding plant growth for optimising their use and management. Agriculture 5:598-625. https://doi.org/10.3390/agriculture5030598.

Difante, G.S., Nascimento Júnior, D., Euclides, V.P.B., Da Silva, S.C., Barbosa, R.A., Gonçalves, W.V., 2009b. Sward structure and nutritive value of Tanzânia guineagrass subject to rotational stocking managements. Rev. Bras. Zootecn. 38 (1):9-19. http://doi.org/10.1590/S1516$\underline{35982009000100002 .}$.

Foley, J.J.A., Ramankutty, N., Brauman, K.A., Cassidy, E.S., Gerber, J.S., Johnston, M.,Mueller, N.D., O'Connell, C., Ray, D.K.,West, P.C., Balzer, C., Bennett, E.M., Carpenter, S.R., Hill, J., Monfreda, C., Polasky, S., Rockström, J., Sheehan, J., Siebert, S., Tilman, D., Zaks, D.P.M., 2011. Solutions for a cultivated planet. Nature 478:337-342. https://doi.org/10.1038/nature10452.

Garnett, T., Godfray, H.C.J., 2012. Sustainable Intensification in Agriculture: Navigating a Course through Competing Food System Priorities. Food Climate Research Network and the Oxford Martin Programme on the Future of Food. University of Oxford, Oxford. https://www.fcrn.org.uk/sites/default/files/SI_report_final.pdf.

Geremia, E.V., Pereira, L.E.T., Paiva, A.J., Oliveira, L.P., Da Silva, S.C., 2014. Intake rate and nutritive value of elephant grass cv. Napier subjected to strategies of rotational stocking management. Trop. Grassl.-Forrajes Trop. 2:51-52. https://doi.org/10.17138/tgft(2)51-52.

Giacomini, A.A., Da Silva, S.C., Sarmento, D.O.L., Zeferino, C.V., Souza Jr., S.J., Trindade, J.K., Guarda, V.D., Nascimento Jr., D., 2009. Growth of marandu palisadegrass subjected to strategies of intermittent stocking. Sci. Agric. 66:733-741. http://doi.org/10.1590/S010390162009000600003. 
Gimenes, F.M.A., Da Silva, S.C., Fialho, C.A., Gomes, M.B., Berndt, A., Gerdes, L., Colozza, M.T., 2011. Ganho de peso e produtividade animal em capim-marandu sob pastejo rotativo e adubação nitrogenada. Pesq. Agropec. Bras. 46:751-759. https://doi.org/10.1590/S0100204X2011000700011.

Godfray, H., Beddington, J.R., Crute, I.R., Haddad, L., Lawrence, D., Muir, J.F., Pretty, J., Robinson, S., Thomas, S.M., Toulmin, C., 2010. Food security: the challenge of feeding 9 billion people. Science 327:812-818. https://doi.org/10.1126/science.1185383.

Lessa, A.C.R., Madari, B.E., Paredes, D.S., Boddey, R.M., Urquiaga, S., Jantalia, C.P., Alves, B.J., 2014. Bovine urine and dung deposited on Brazilian savannah pastures contribute differently to direct and indirect soil nitrous oxide emissions. Agric. Ecosyst. Environ. 190:104-111. https://doi.org/10.1016/j.agee.2014.01.010.

Luo, J., Balvert, S.F., Wise, B., Welten, B., Ledgard, S.F., de Klein, C.A.M., Lindsey, S., Judge, A. Using alternative forage species to reduce emissions of the greenhouse gas nitrous oxide from cattle urine deposited onto soil. Sci. Total Environ. 610-611:1271-1280. https://doi.org/10.1016/j.scitotenv.2017.08.186.

Macdonald, K.A., Penno, J.W., Lancaster, J.A.S., Bryant, A.M., Kidd, J.M., Roche, J.R., 2017. Production and economic responses to intensification of pasture-based dairy production systems. J. Dairy Sci. 100:6602-6619. https://doi.org/10.3168/jds.2016-12497.

Muñoz, C., Letelier, P.A., Ungerfeld, E.M., Morales, J.M., Hube, S., Pérez-Prieto, L.A., 2016. Effects of pre grazing herbage mass in late spring on enteric methane emissions, dry matter intake, and milk production of dairy cows. J. Dairy Sci. 99:7945-7955. https://doi.org/10.3168/jds.2016$\underline{10919 .}$.

Nascimento, C.F.M., Berndt, A., Romero Solorzano, L.A., Meyer, P.M., Frighetto, R.T.S., Demarchi, J.J.A.A., Rodrigues, P.H.M., 2016. Methane emission of cattle fed Urochloa brizantha hay harvested at different stages. J. Agric. Sci. 8:163-174. http://dx.doi.org/10.5539/jas.v8n1p163.

Pereira, L.E.T., Paiva, A.J., Geremia, E.V., Da Silva, S.C., 2014. Components of herbage accumulation in elephant grass cvar Napier subjected to strategies of intermittent stocking management. J. Agric. Sci. 152:954-966. https://doi.org/10.1017/S0021859613000695.

Pereira, L.E.T., Paiva, A.J., Geremia, E.V., da Silva, S.C., 2015a. Grazing management and tussock distribution in elephant grass. Grass Forage Sci. 70:1-12. https://doi.org/10.1111/gfs.12137.

Pereira, L.E.T., Paiva, A.J., Geremia, E.V., Da Silva, S.C., 2015b. Regrowth patterns of elephant grass (Pennisetum purpureum Schum.) subjected to strategies of intermittent stocking management. Grass Forage Sci. 70:195-204. https://doi.org/10.1111/gfs.12103.

Pereira, L.E.T., Paiva, A.J., Geremia, E.V., Da Silva, S.C., 2018. Contribution of basal and aerial tillers to sward growth in intermittently stocked elephant grass. Grassl. Sci. 64:108-117. https://doi.org./10.1111/grs.12194. 
Pontes, L.S., Barro, R.S., Savian, J.V., Berndt, A., Moletta, J.L., Porfírio-da-Silva, V., Bayer, C., Carvalho, P.C.F., 2018. Performance and methane emissions by beef heifer grazing in temperate pastures and in integrated crop-livestock systems: The effect of shade and nitrogen fertilization. Agric. Ecosyst. Environ. 253:90-97. https://doi.org/10.1016/j.agee.2017.11.009.

Ramsbottom, G., Horan, B., Berry, D.P., Roche, J.R., 2015. Factors associated with the financial performance of spring-calving, pasture-based dairy farms. J. Dairy Sci. 98:3526-3540. https://doi.org/10.3168/jds.20148516.

Royal Society, 2009. Reaping the Benefits: Science and the Sustainable Intensification of Global Agriculture. The Royal Society, London. https://royalsociety.org/ /media/Royal Society Content/policy/publications/2009/4294967719.pd f.

Sbrissia, A.F., Duchini, P.G., Zanini, G.D., Santos, G.T., Padilha, D.A., Schimitt, D., 2018. Defoliation strategies in pastures submitted to intermittent stocking method: underlying mechanisms buffering forage accumulation over a range of grazing heights. Crop Sci. 58:1-10. https://doi.org./10.2135/cropsci2017.07.0447.

Silveira, M.C.T., Da Silva, S.C., Souza Jr., S.J., Barbero, L.M., Rodrigues, C.S., Limão, V.A., Pena, K.S., Nascimento Jr., D., 2013. Herbage accumulation and grazing losses on Mulato grass subjected to strategies of rotational stocking management. Sci. Agric. 70:242-249. https://doi.org/10.1590/S0103-90162013000400004.

Silveira, M.C.T., Nascimento Jr., D., Rodrigues, C.S., Pena, K.S., Souza Jr., S.J., Barbero, L.M., Limão, V.A., Euclides, V.P.B., Da Silva, S.C., 2016. Forage sward structure of Mulato grass (Brachiaria hybrid ssp.) subjected to rotational stocking strategies. Aust. J. Crop Sci. 10(6):864873. https://doi.org/10.21475/ajcs.2016.10.06.p7568.

Trindade, J.K., Da Silva, S.C., Souza Jr, S.J., Giacomini, A.A., Zeferino, C.V., Guarda, V.D.A., Carvalho, P.C.F., 2007. Composição morfológica da forragem consumida por bovinos de corte durante o rebaixamento do capim-marandu submetido a estratégias de pastejo rotativo. Pesq. Agropec. Bras. 42:883-890. https://doi.org/10.1590/S0100204X2007000600016.

Zanini, G.D., Santos, G.T., Schmitt, D., Padilha, D.S., Sbrissia, A.F., 2012. Distribuição de colmo na estrutura vertical de pastos de capim Aruana e azevém anual submetidos a pastejo intermitente por ovinos. Cienc. Rural. 42 (5):882-887. http://doi.org/10.1590/S0103-84782012000500020. 


\section{CONCLUSIONS}

Strategic grazing management represented by the LI $95 \%$ pre-grazing target associated with moderate severity of defoliation (50\% of the pre-grazing sward surface height) is an environmentally friendly practice that improves the use efficiency of allocated resources through optimization of processes involving plant, ruminant and their interface, and enhances milk production efficiency of tropical pasture-based systems. In addition, daily allocation of herd to new paddock in the afternoon might increase $\mathrm{N}$ partitioning to protein yield, and decrease excretion of urea $\mathrm{N}$ in milk. The association of $\mathrm{LI}_{95 \%}$ pre-grazing target and afternoon allocation could bring economic, productive and environmental benefits towards sustainable intensification of tropical pasture-based systems. 